\title{
GECCO 2018 Tutorial on Evolutionary Multiobjective Optimization
}

\author{
Dimo Brockhoff
}

dimo.brockhoff@inria.fr

updated slides will be available at http://www.cmap.polytechnique. fr/ dimo.brockhoff/
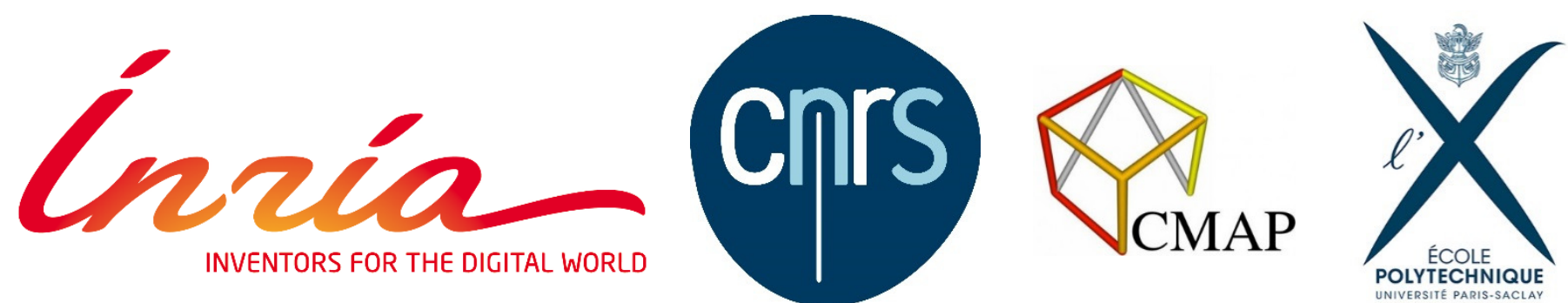

Permission to make digital or hard copies of part or all of this work for personal or classroom use is granted without fee provided that copies are not made or distributed for profit or commercial advantage and that copies bear this notice and the full citation on the first page. Copyrights for third-party components of this work must be honored. For all other uses, contact the Owner/Author.

GECCO'18 Companion, July 15-19, 2018, Kyoto, Japan (C) 2018 Copyright is held by the owner/author(s).

ACM ISBN 978-1-4503-5764-7/18/07.

https://doi.org/10.1145/3205651.3207864 


\section{A Brief Introduction to Multiobjective Optimization}

\section{Multiobjective Optimization}

Multiple objectives that have to be optimized simultaneously

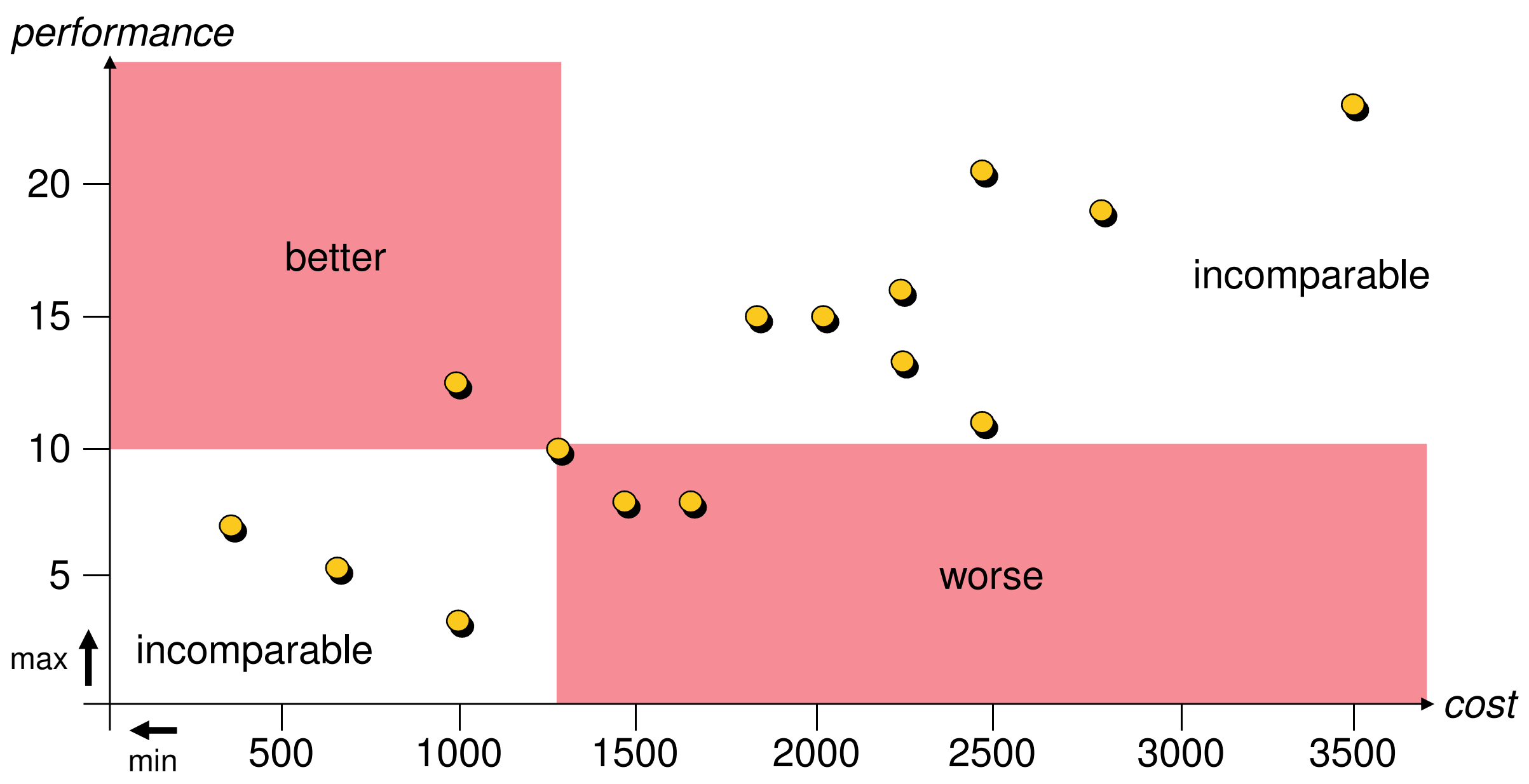




\section{A Brief Introduction to Multiobjective Optimization}

Observations: (1) there is no single optimal solution, but

2 some solutions (o) are better than others (o)

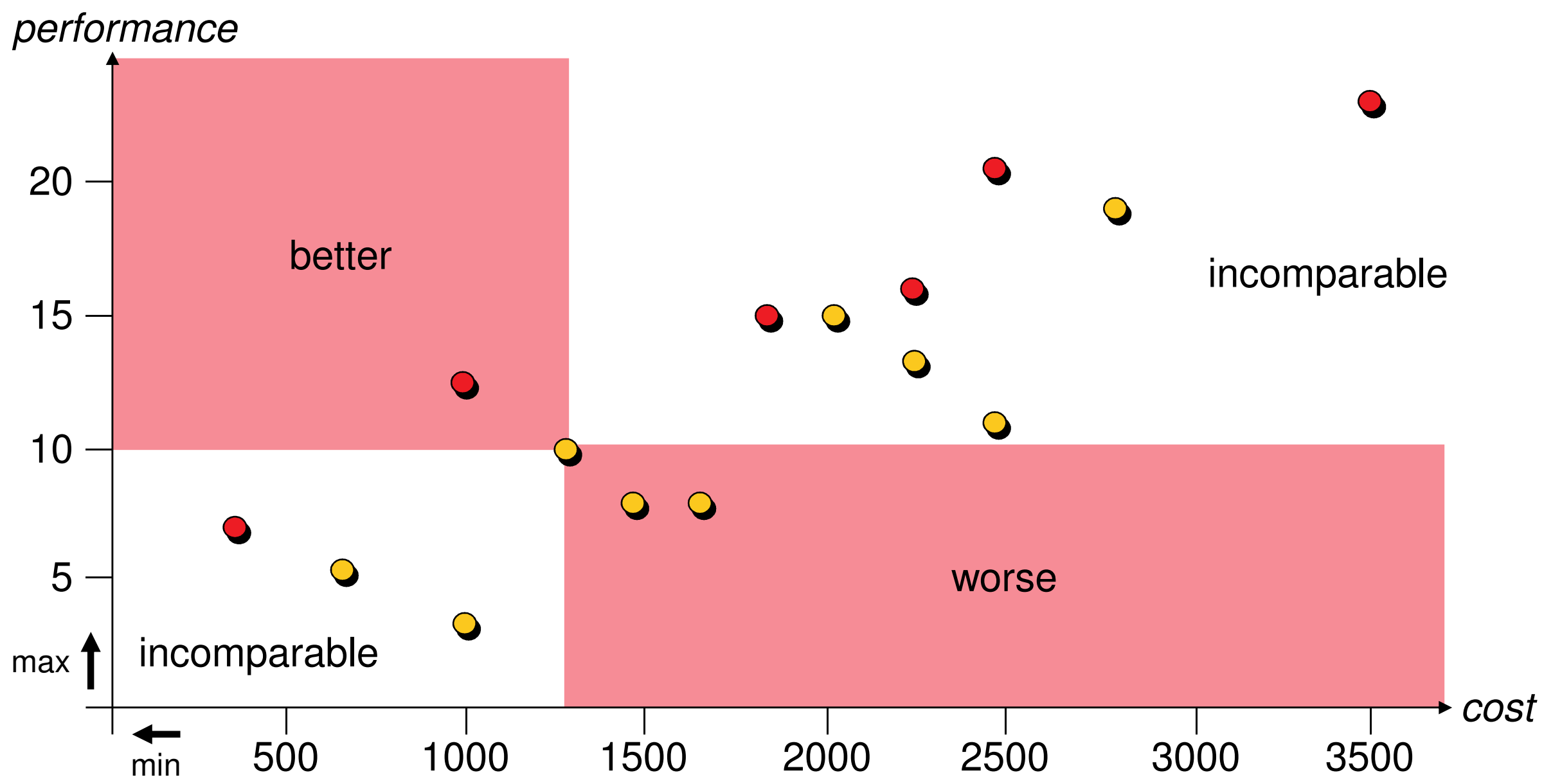




\section{A Brief Introduction to Multiobjective Optimization}

$u$ weakly Pareto dominates $v\left(u \leqslant_{\text {par }} v\right): \quad \forall 1 \leq i \leq k: f_{i}(u) \leq f_{i}(v)$

$u$ Pareto dominates $v\left(u<_{\text {par }} v\right): u \leqslant$ par $v \wedge v \nless_{\text {par }} u$

performance

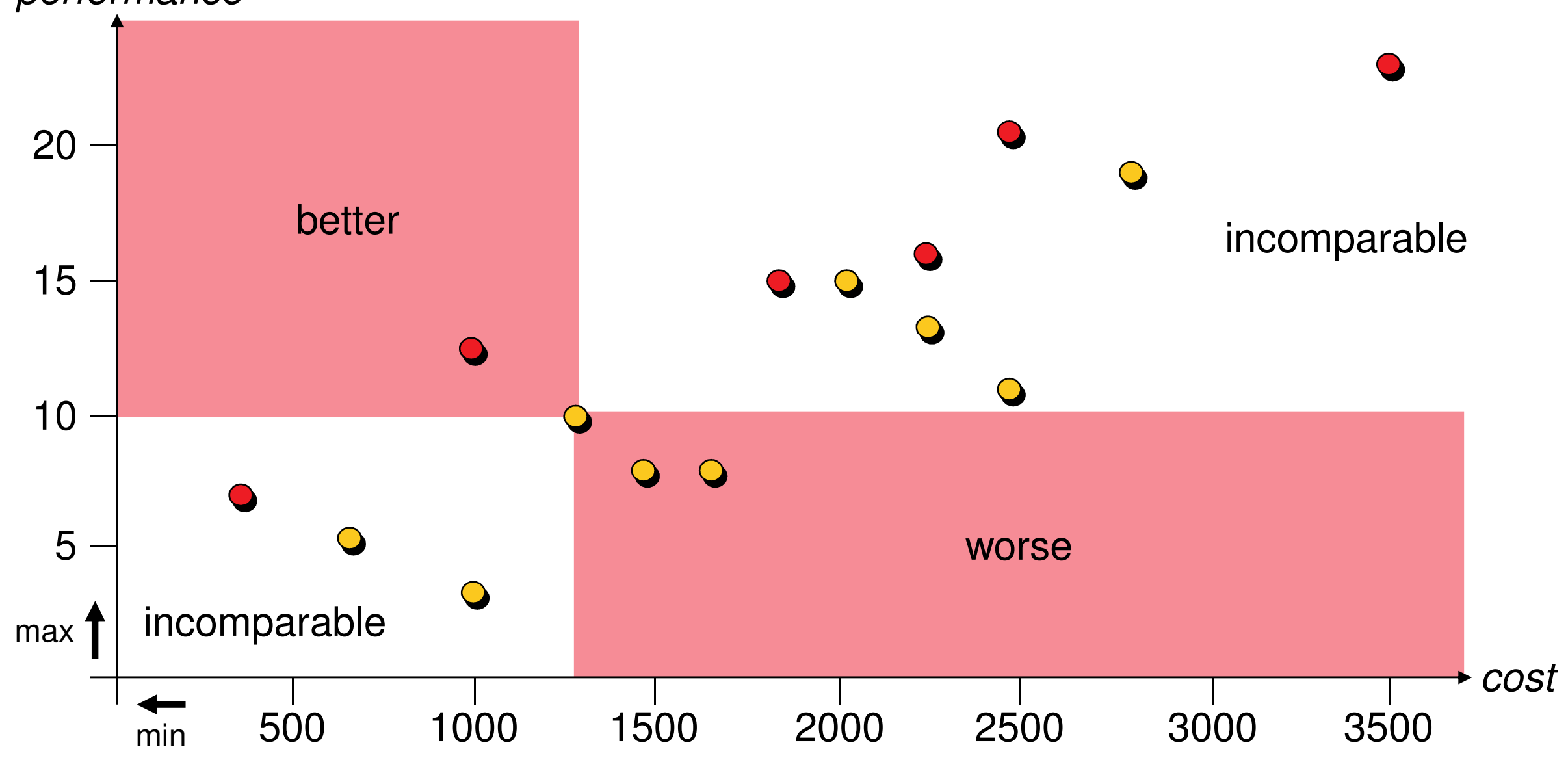




\section{A Brief Introduction to Multiobjective Optimization}

$u$ weakly Pareto dominates $v\left(u \leqslant_{\text {par }} v\right): \quad \forall 1 \leq i \leq k: f_{i}(u) \leq f_{i}(v)$

$u$ Pareto dominates $v\left(u<_{\text {par }} v\right): u \leqslant$ par $v \wedge v \nless_{\text {par }} u$

performance

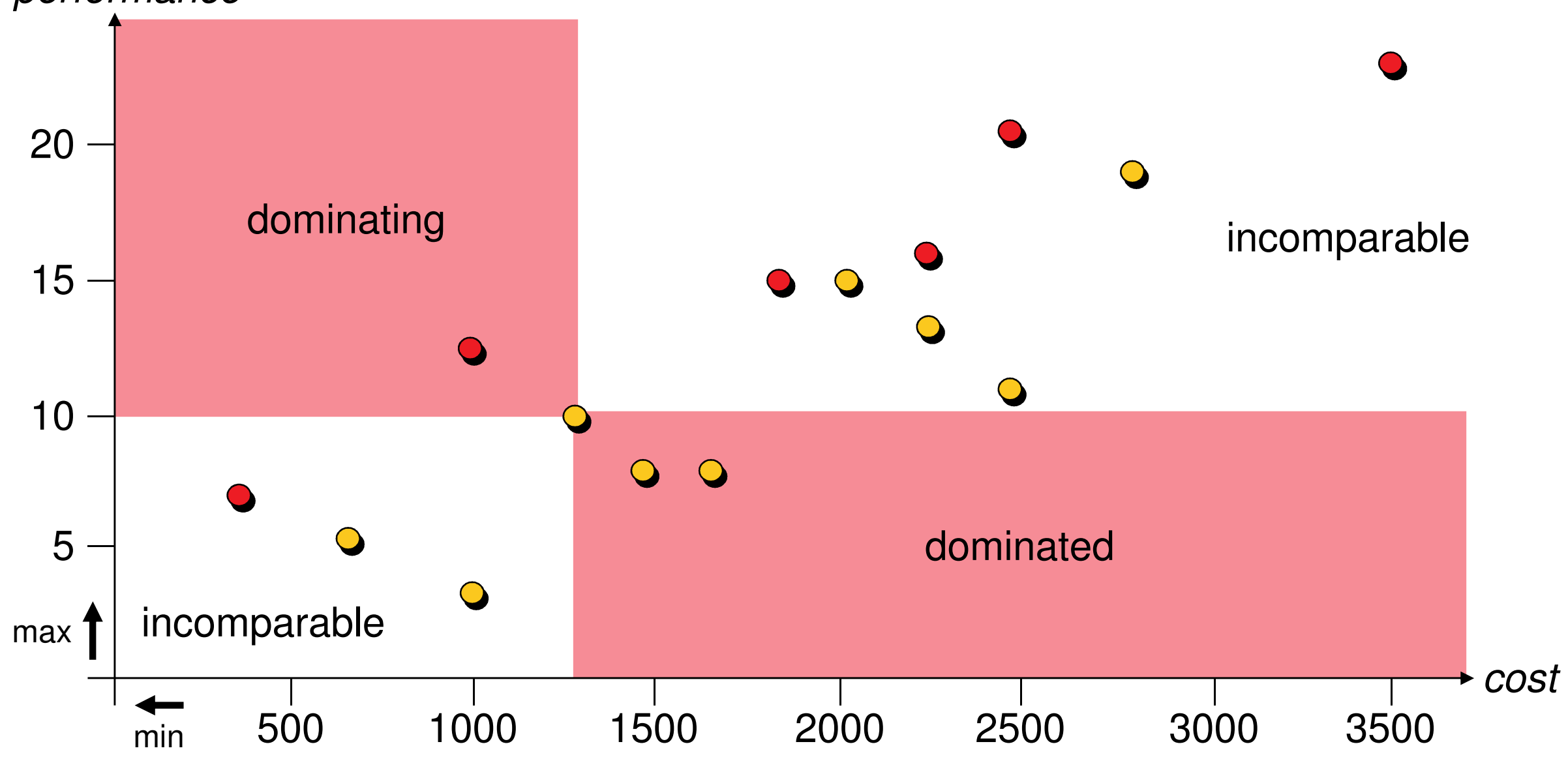




\section{A Brief Introduction to Multiobjective Optimization}

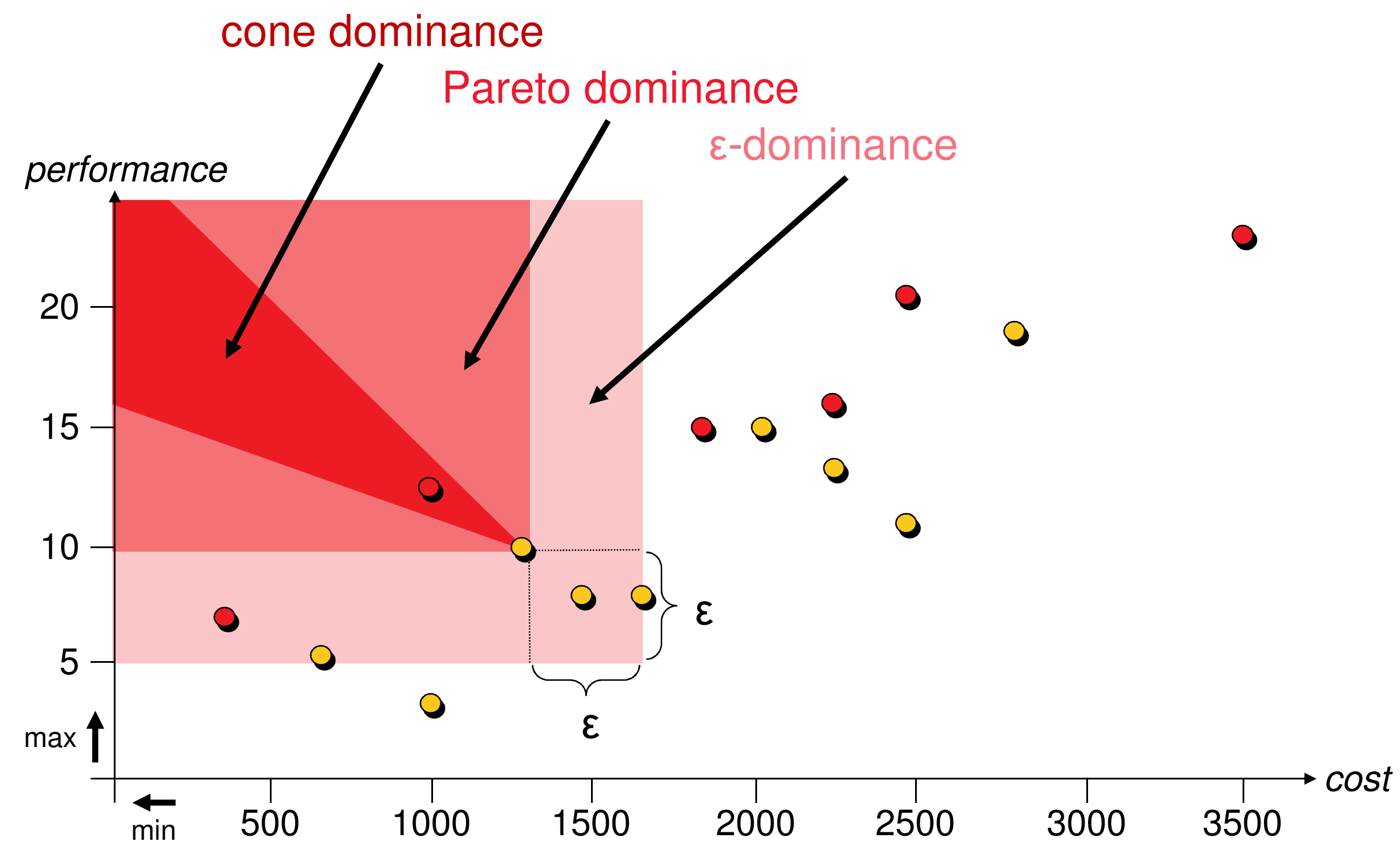




\section{A Brief Introduction to Multiobjective Optimization}

Pareto set: set of all non-dominated solutions (decision space) Pareto front: its image in the objective space

performance

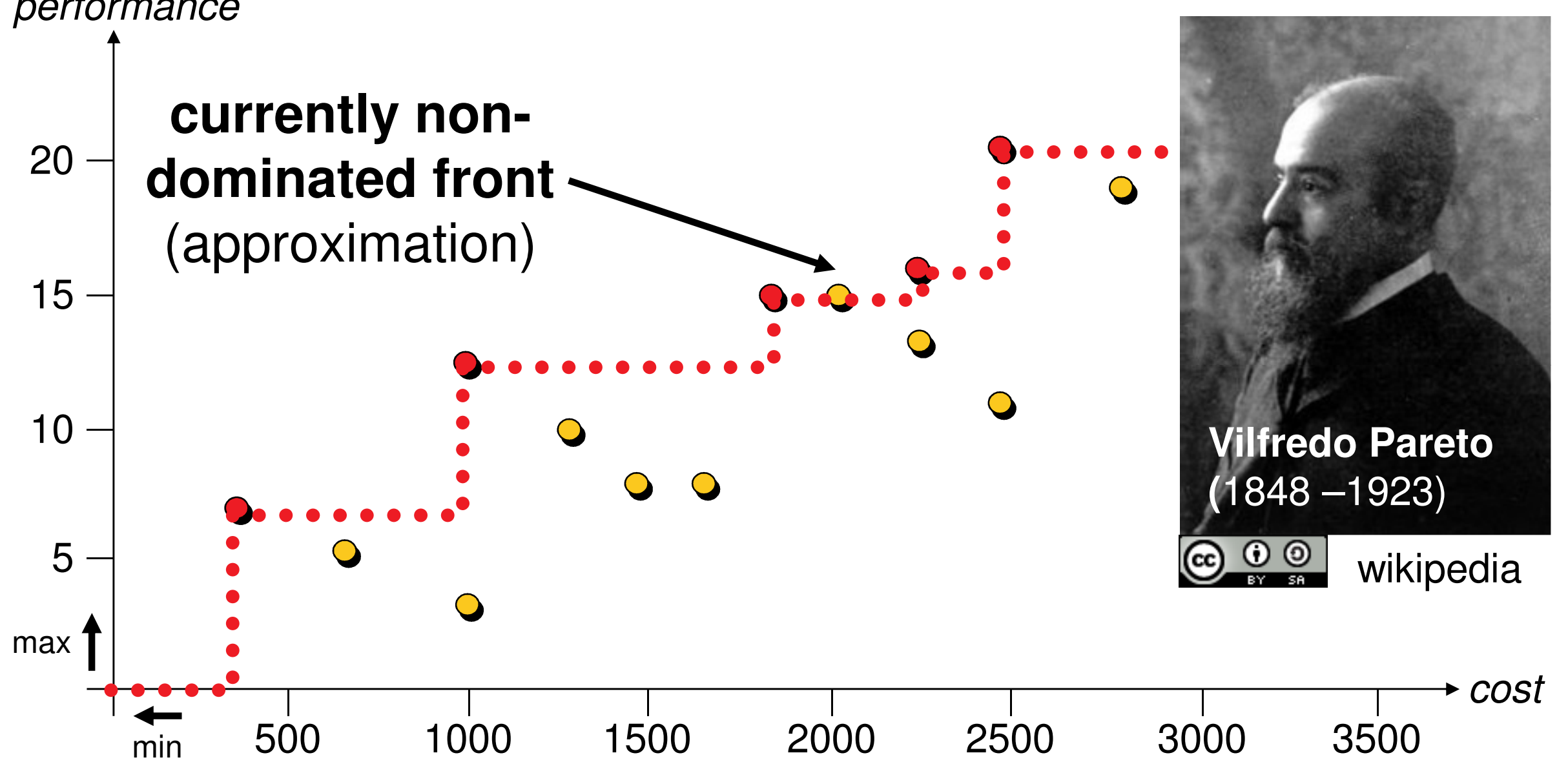




\section{A Brief Introduction to Multiobjective Optimization}

Pareto set: set of all non-dominated solutions (decision space) Pareto front: its image in the objective space

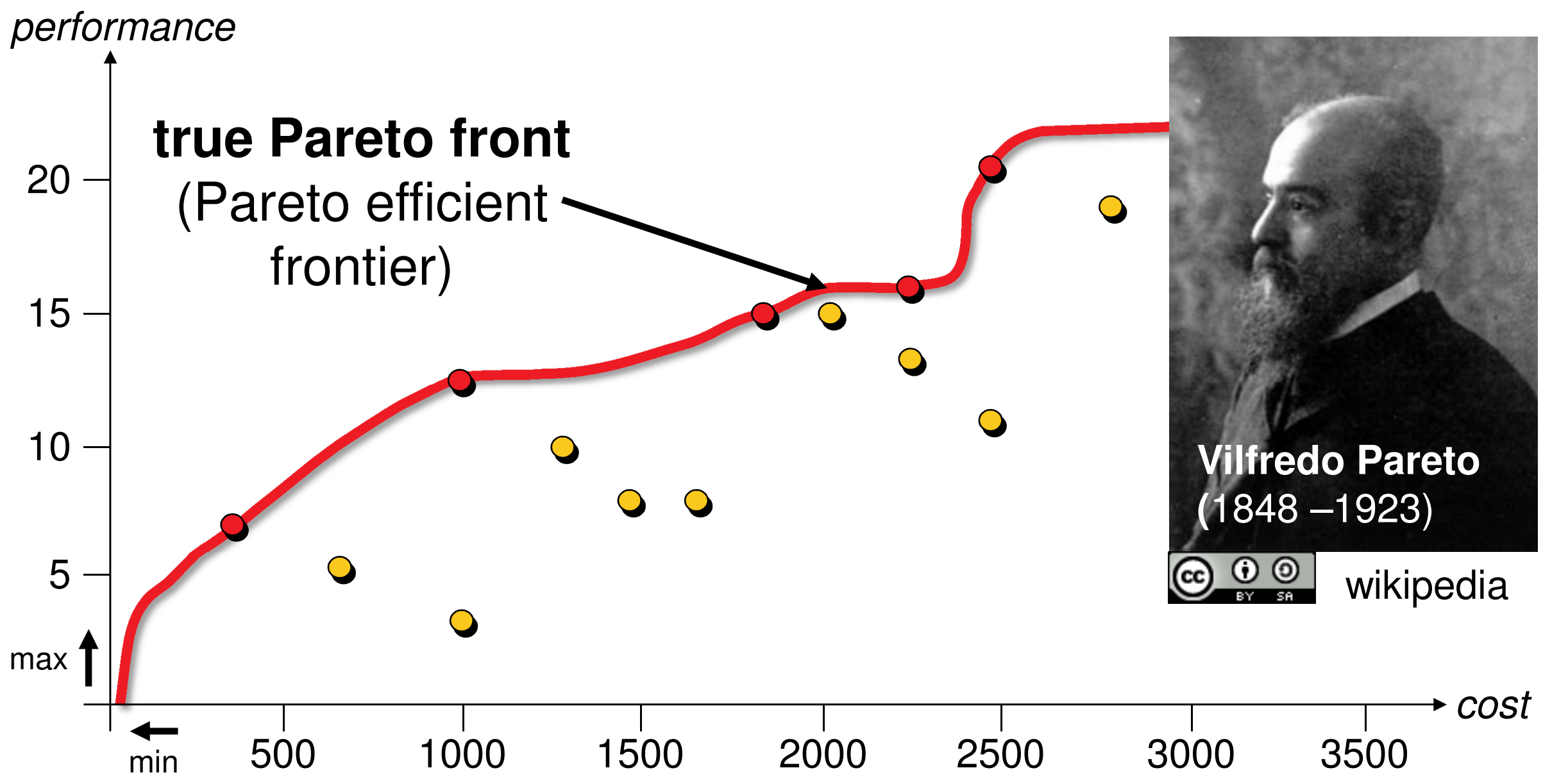




\section{A Brief Introduction to Multiobjective Optimization}

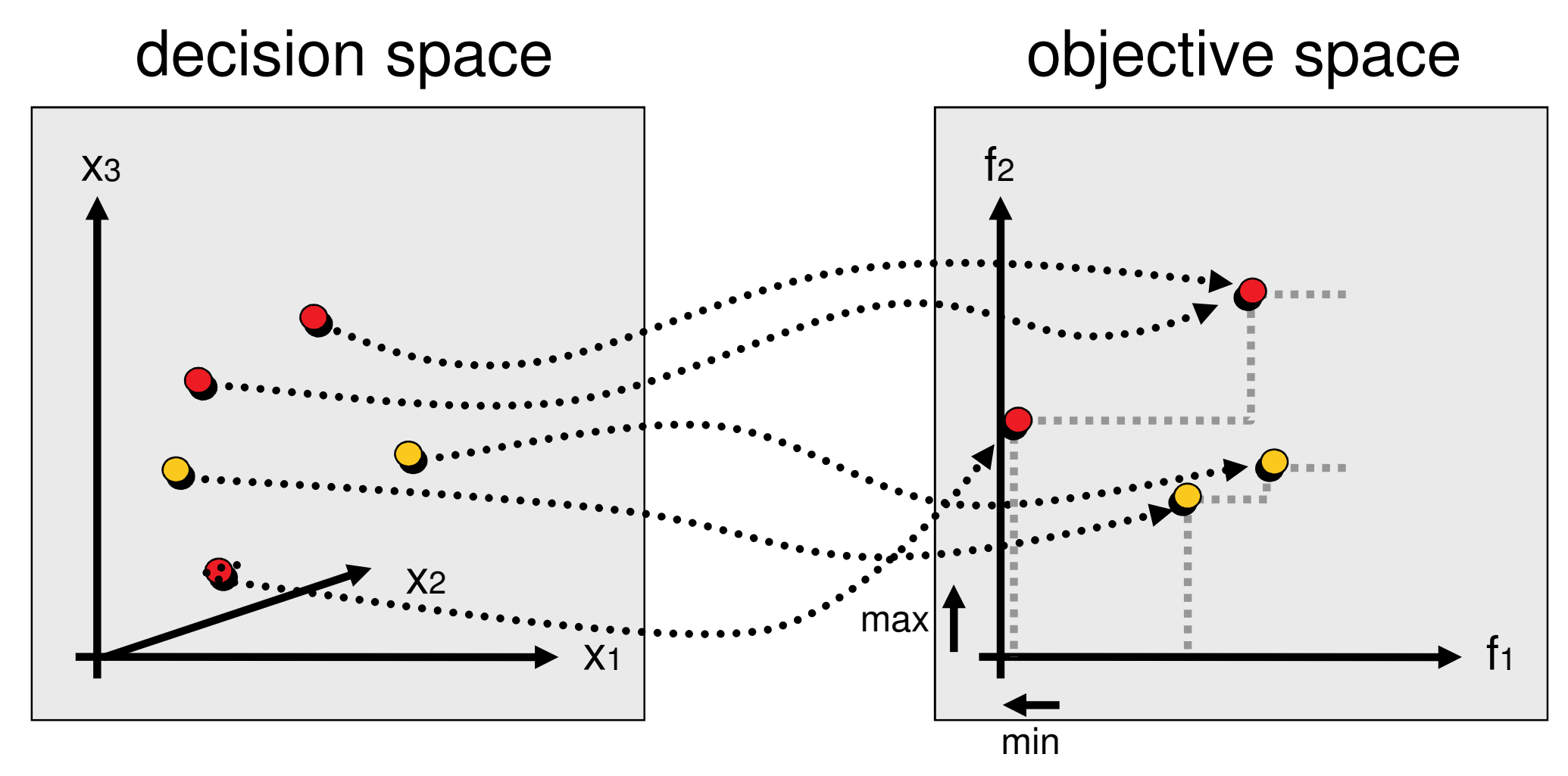

solution of Pareto-optimal set $\bullet$ vector of Pareto-optimal front non-optimal decision vector $\bigcirc$ non-optimal objective vector 


\section{A Brief Introduction to Multiobjective Optimization}

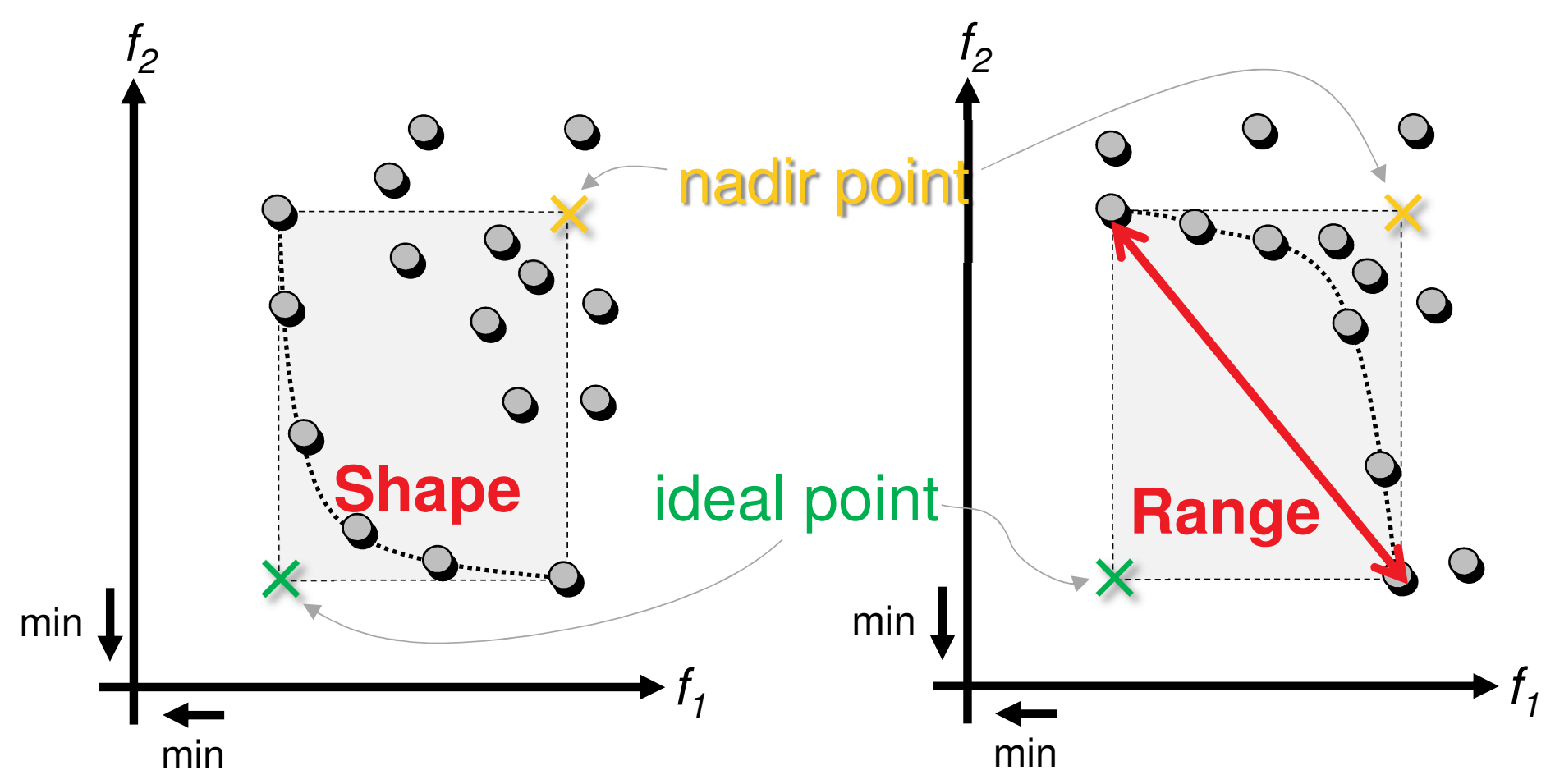

$\left.\begin{array}{l}\text { ideal point: best values } \\ \text { nadir point: worst values }\end{array}\right\}$ obtained for Pareto-optimal points 


\section{Optimization vs. Decision Making}

\section{Multiobjective Optimization}

combination of optimization of a set and a decision for a solution

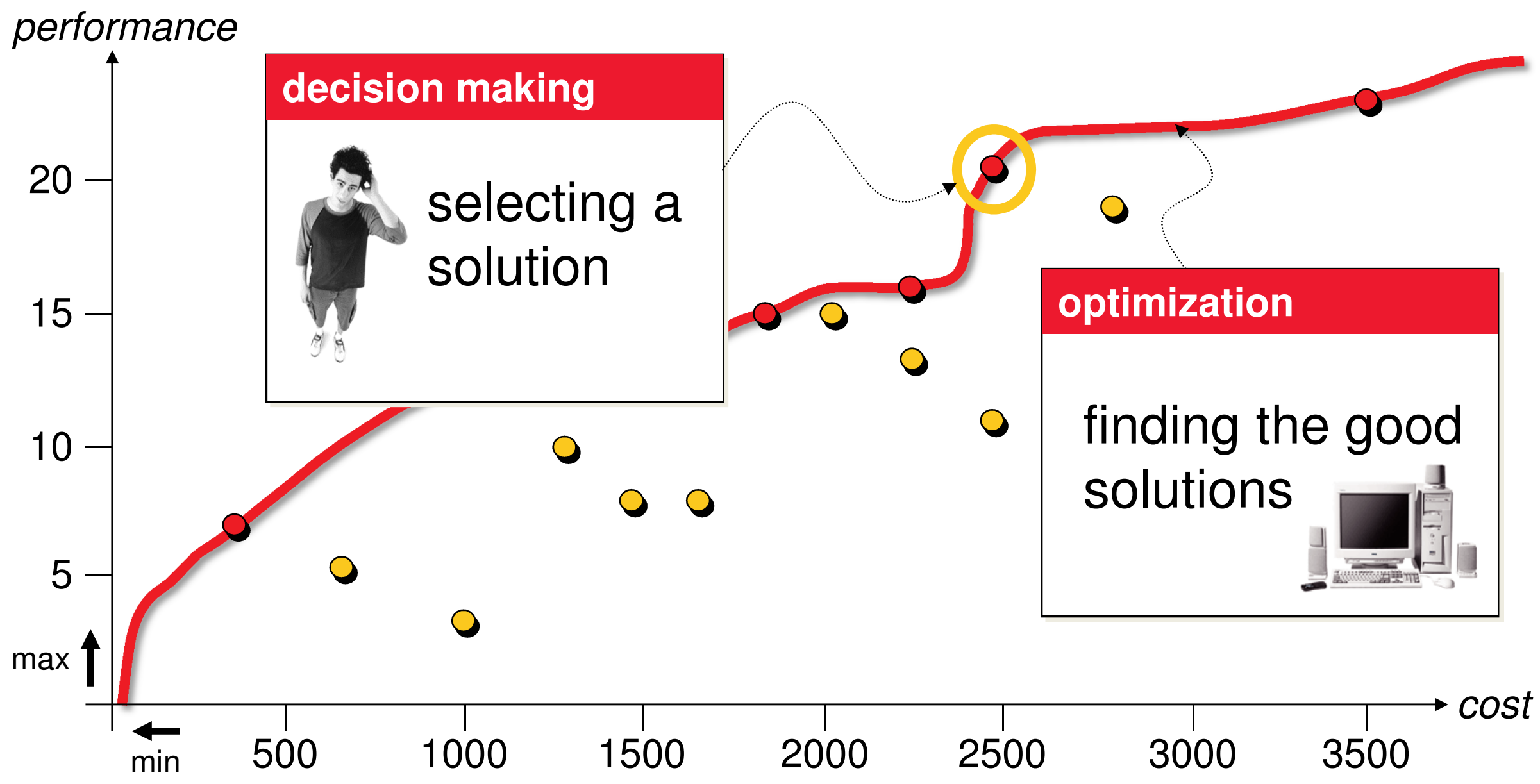




\section{Selecting a Solution: Examples}

Possible (1) ranking: performance more important than cost Approaches:

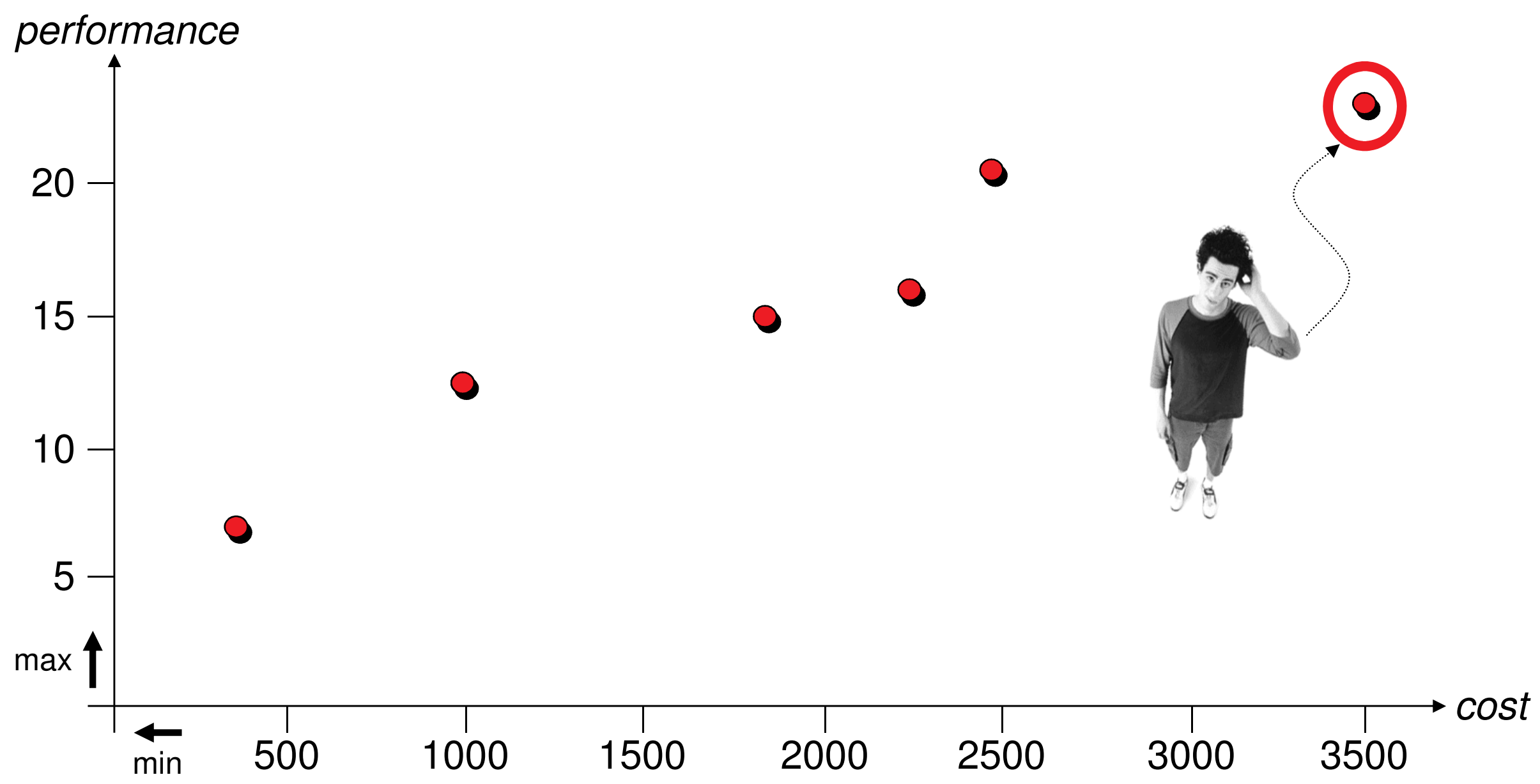




\section{Selecting a Solution: Examples}

Possible (1) ranking: performance more important than cost Approaches: 2 constraints: cost must not exceed 2400

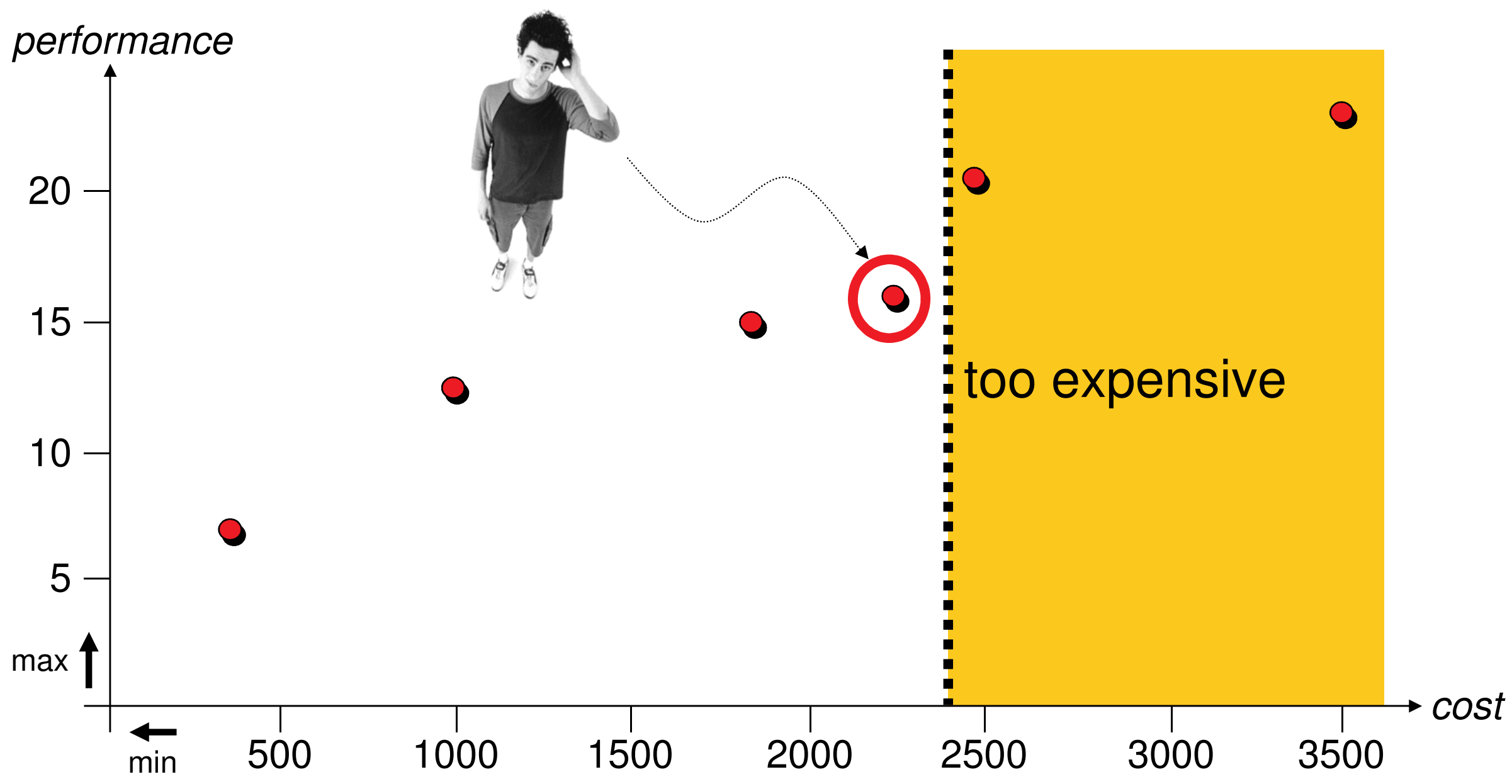




\section{When to Make the Decision}

\section{Before Optimization:}

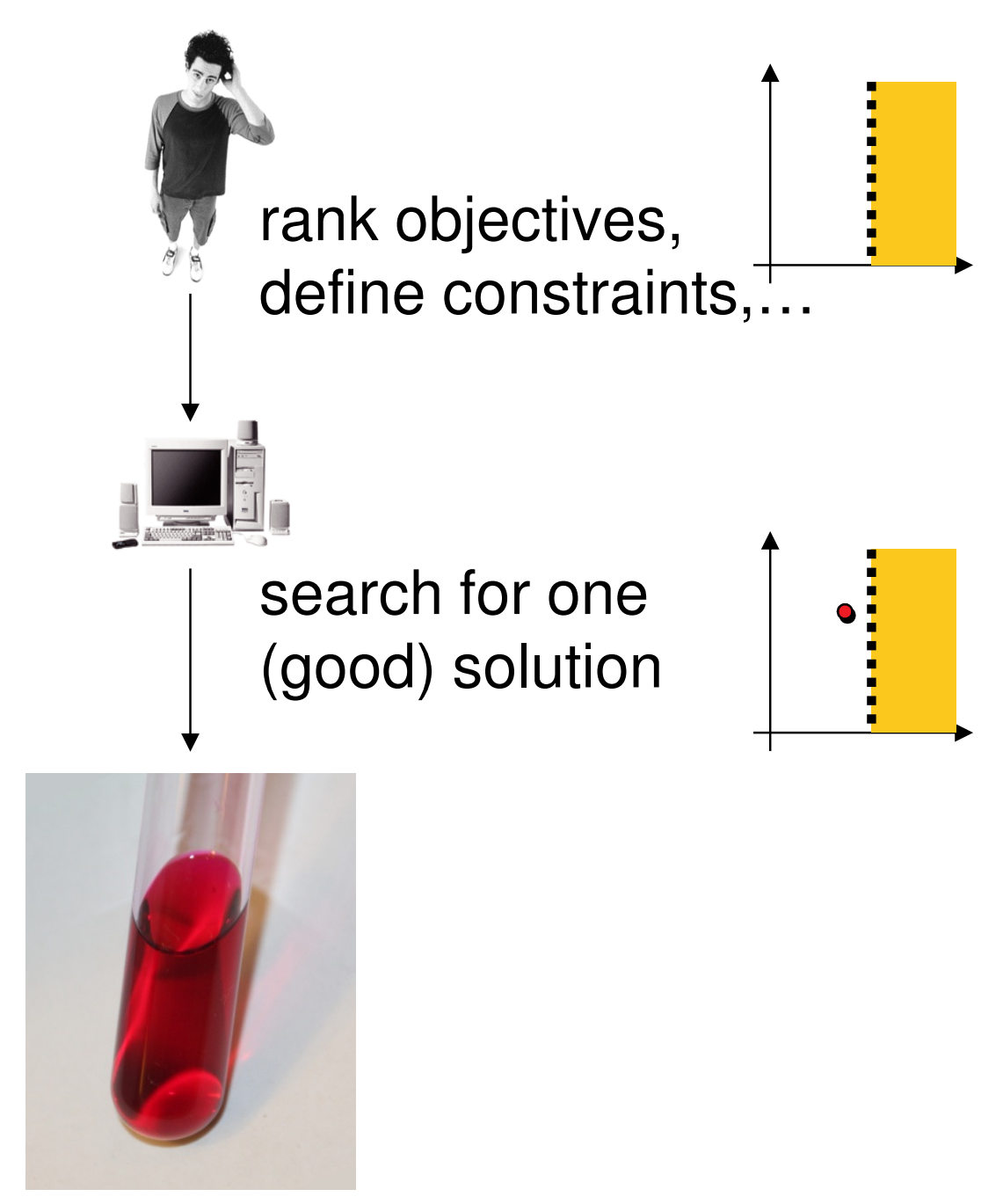




\section{When to Make the Decision}

\section{Before Optimization:}

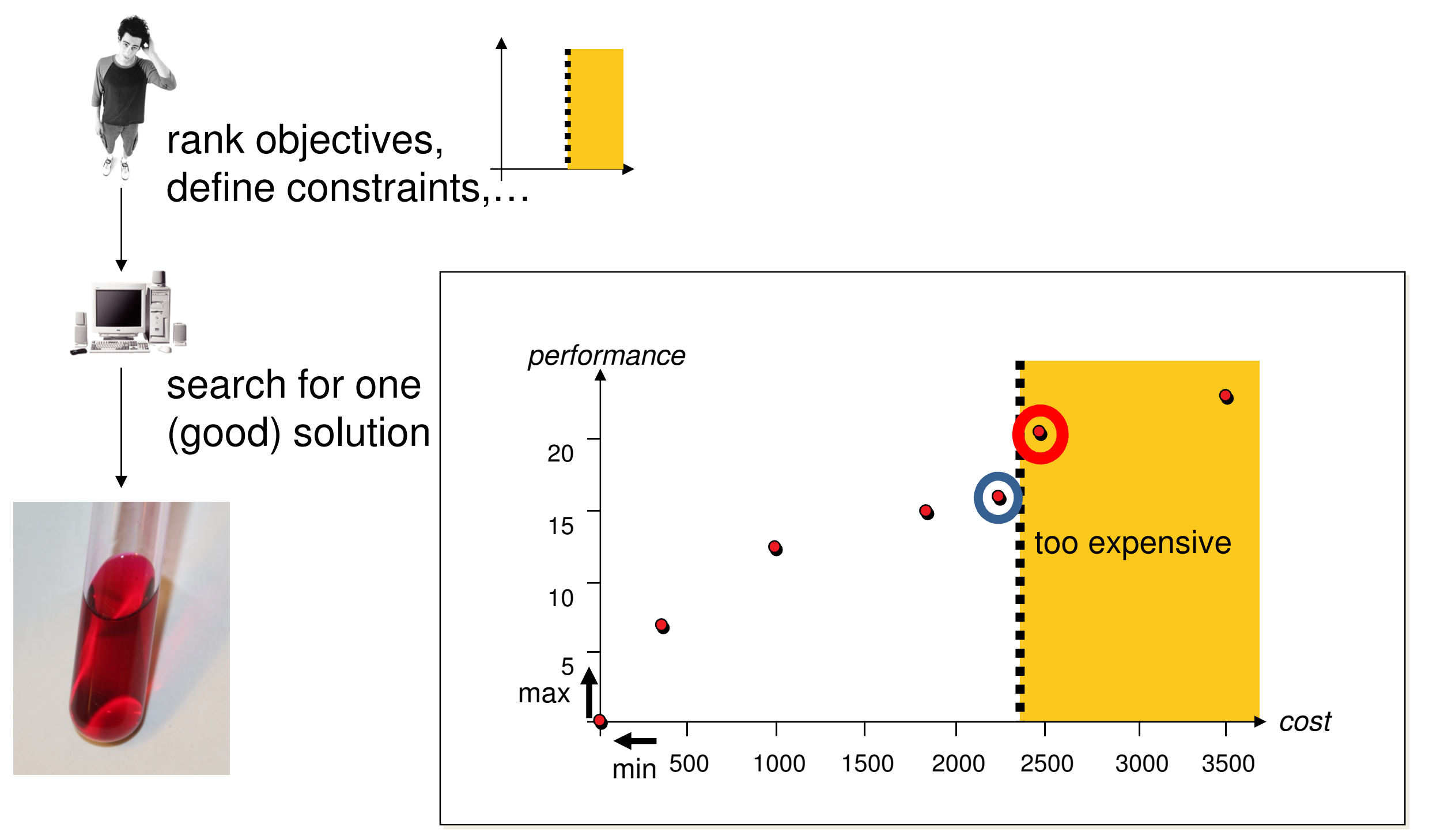




\section{When to Make the Decision}

\section{Before Optimization:}

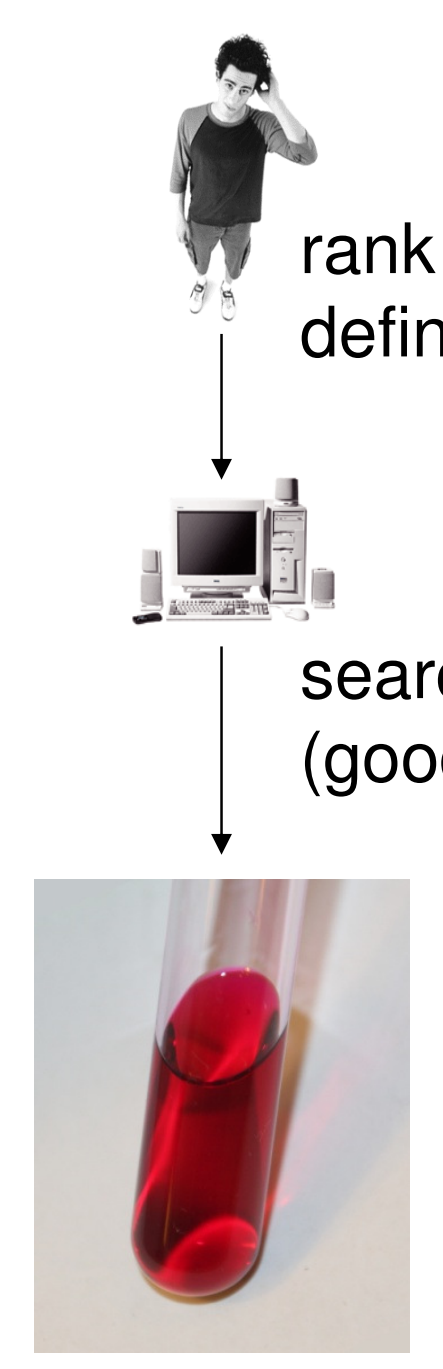

\section{After Optimization:}

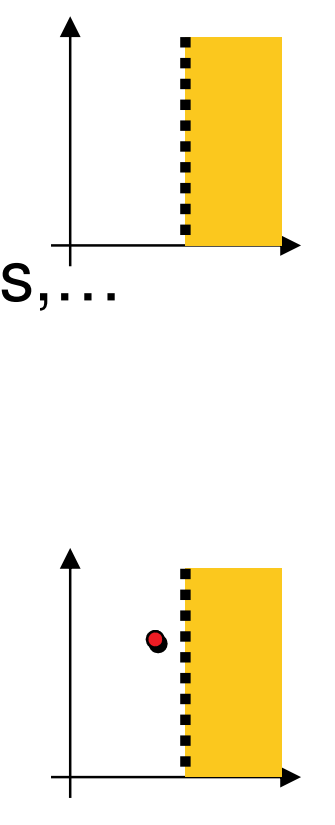

search for a set of (good) solutions

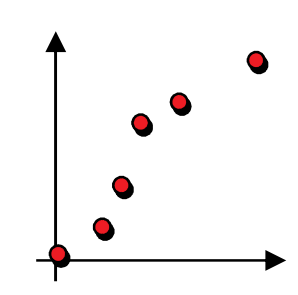

select one solution considering constraints, etc.

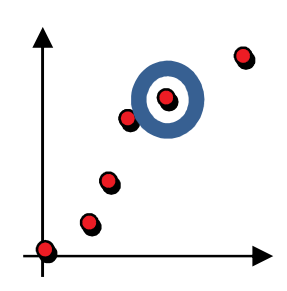




\section{When to Make the Decision}

\section{Before Optimization:}

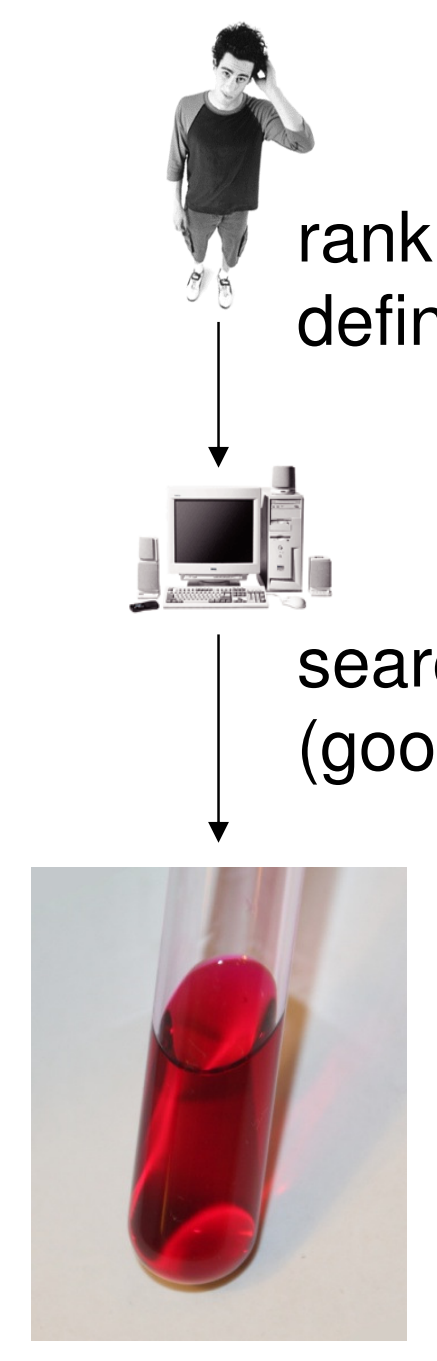

\section{After Optimization:}

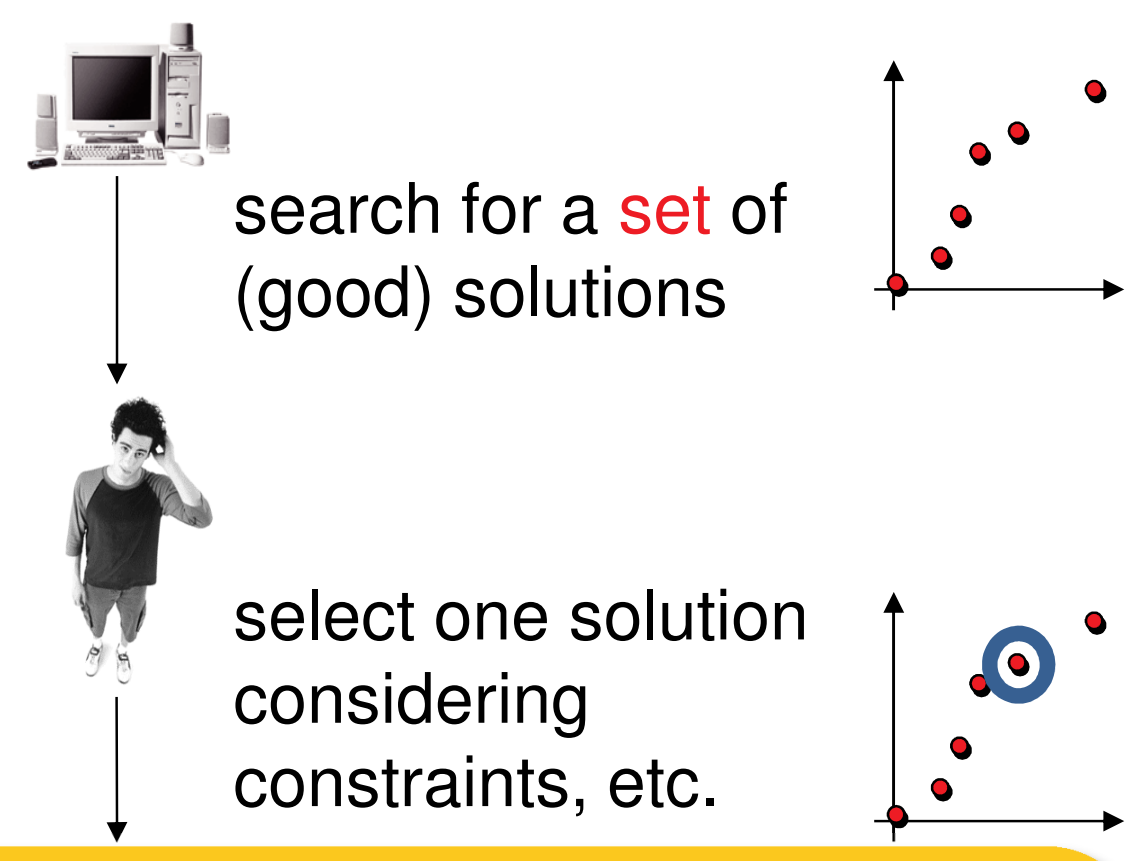

Focus: learning about a problem

- trade-off surface

- interactions among criteria

- structural information

- also: interactive optimization 


\section{Two Communities...}

- established field (beginning in 1950s/1960s)

- bi-annual conferences since 1975

- background in economics, math, management and social sciences

- focus on optimization and decision making
- quite young field (first papers in mid 1980s)

- bi-annual conference since 2001

- background in computer science, applied math and engineering

- focus on optimization algorithms 


\section{...Slowly Merge Into One}

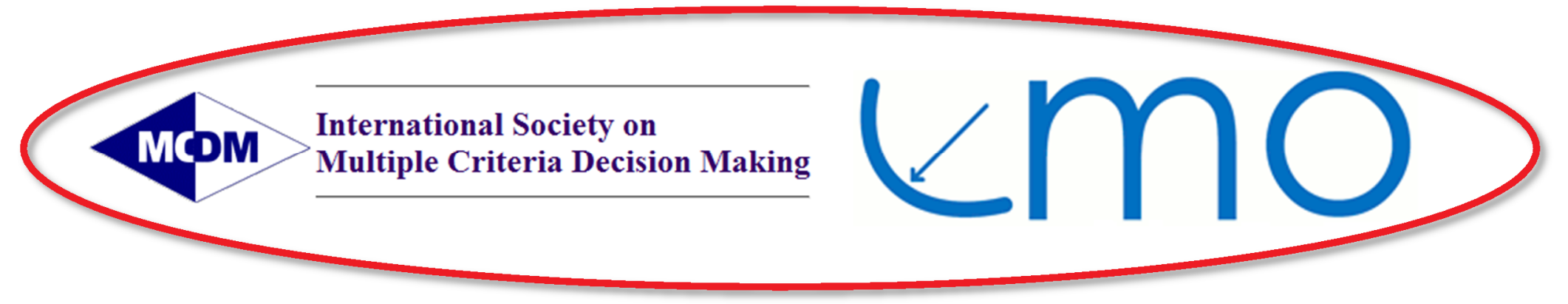

- MCDM track at EMO conference since 2009

- special sessions on EMO at the MCDM conference since 2008

- joint Dagstuhl seminars since 2004 


\section{One of the Main Differences}

\section{Blackbox optimization}

$$
x \in X \longrightarrow \underset{\substack{\boldsymbol{f} \\ \text { only mild assumptions }}}{\longrightarrow}\left(f_{1}(x), \ldots, f_{k}(x)\right)
$$

$\rightarrow$ EMO therefore well-suited for real-world engineering problems

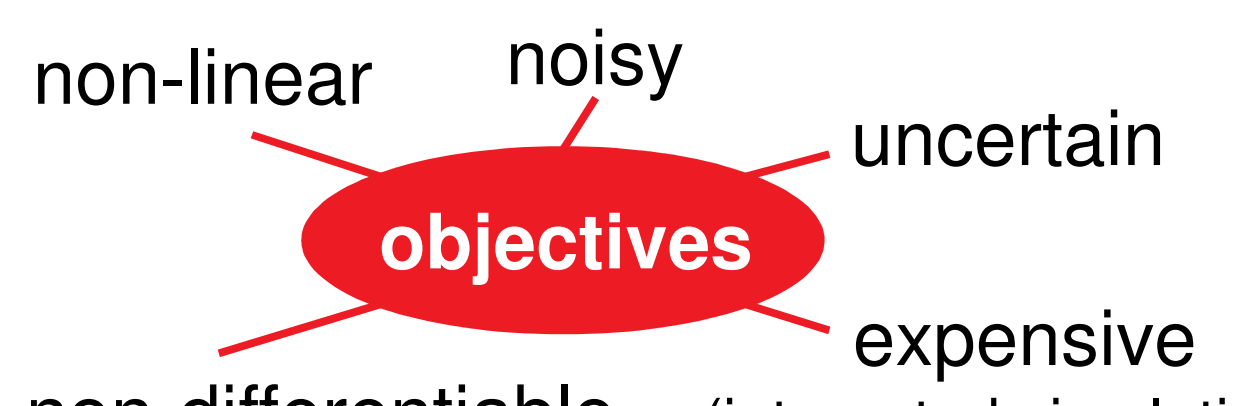

non-differentiable (integrated simulations, real experiments) many objectives

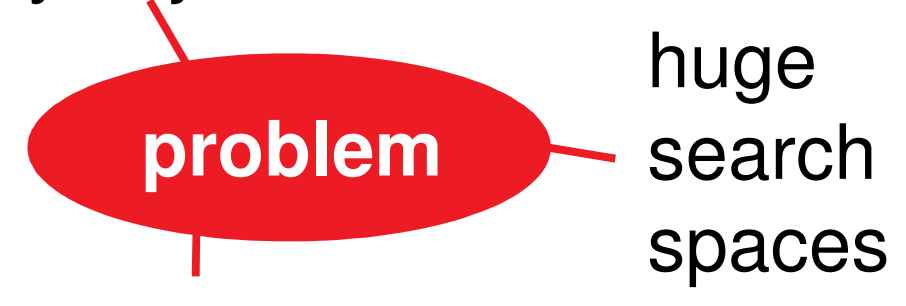

many constraints 


\section{The Other Main Difference}

\section{Evolutionary Multiobjective Optimization}

- set-based algorithms

- therefore possible to approximate the Pareto front in one run
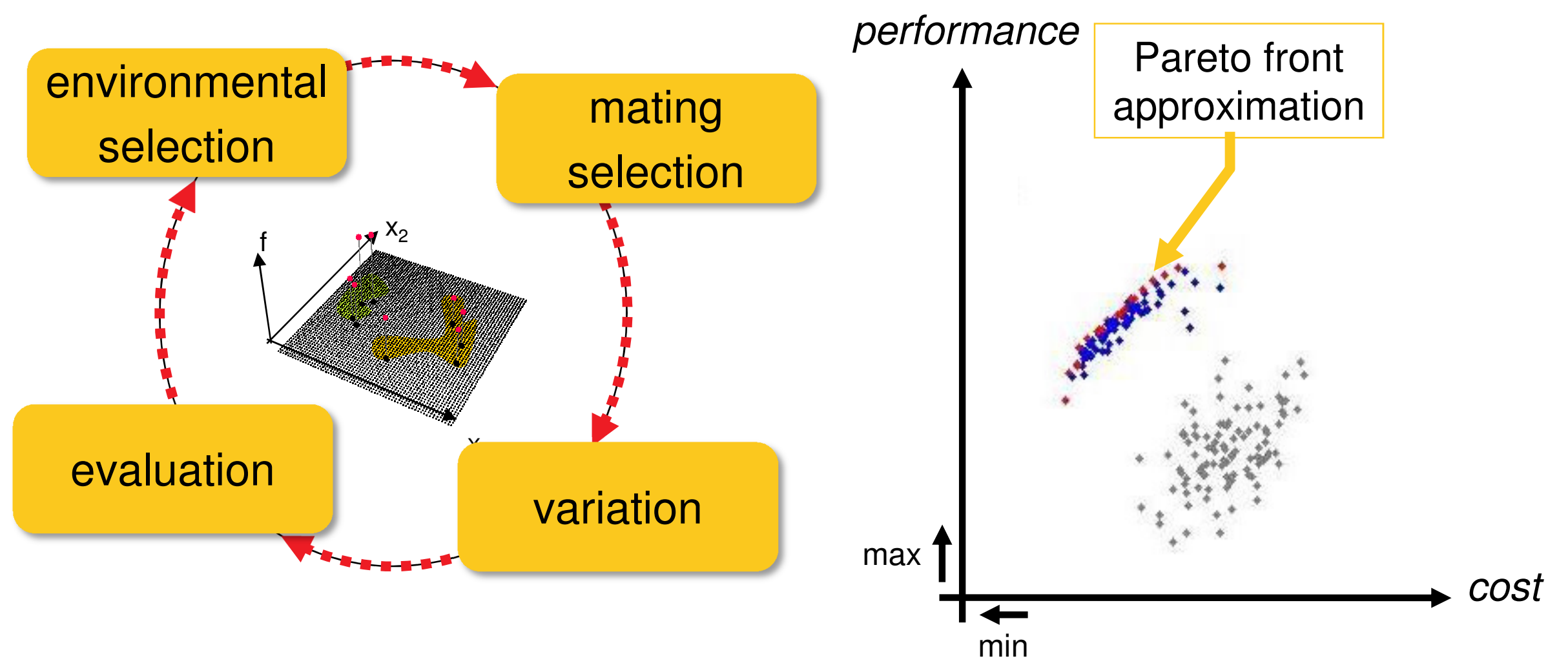


\section{Multiobjectivization}

Some problems are easier to solve in a multiobjective scenario

example: TSP

[Knowles et al. 2001]

\section{Multiobjectivization}

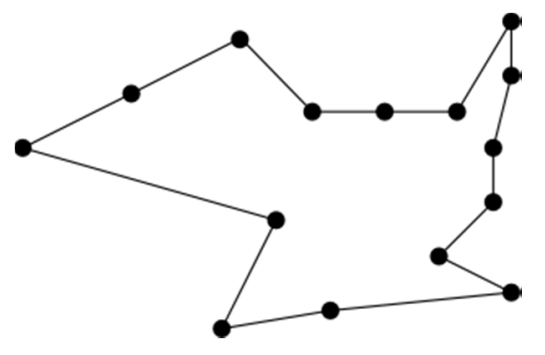

$$
\pi \in S_{n} \rightarrow f(\pi)
$$

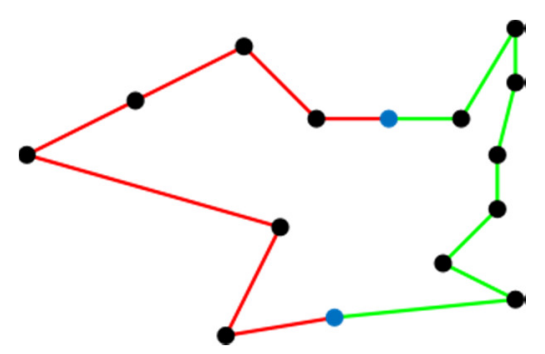

$\pi \in S_{n} \rightarrow\left(f_{1}(\pi, a, b), f_{2}(\pi, a, b)\right)$

by addition of new "helper objectives" [Jensen 2004]

job-shop scheduling [Jensen 2004], frame structural design

[Greiner et al. 2007], VRP [Watanabe and Sakakibara 2007], ...

by decomposition of the single objective

TSP [Knowles et al. 2001], minimum spanning trees [Neumann and

Wegener 2006], protein structure prediction [Handl et al. 2008a], ...

also backed up by theory e.g. [Brockhoff et al. 2009, Handl et al. 2008b] related to constrained and multimodal single-objective optimization

see also this recent overview: [Segura et al. 2013] 


\section{Innovization}

Often innovative design principles among solutions are found

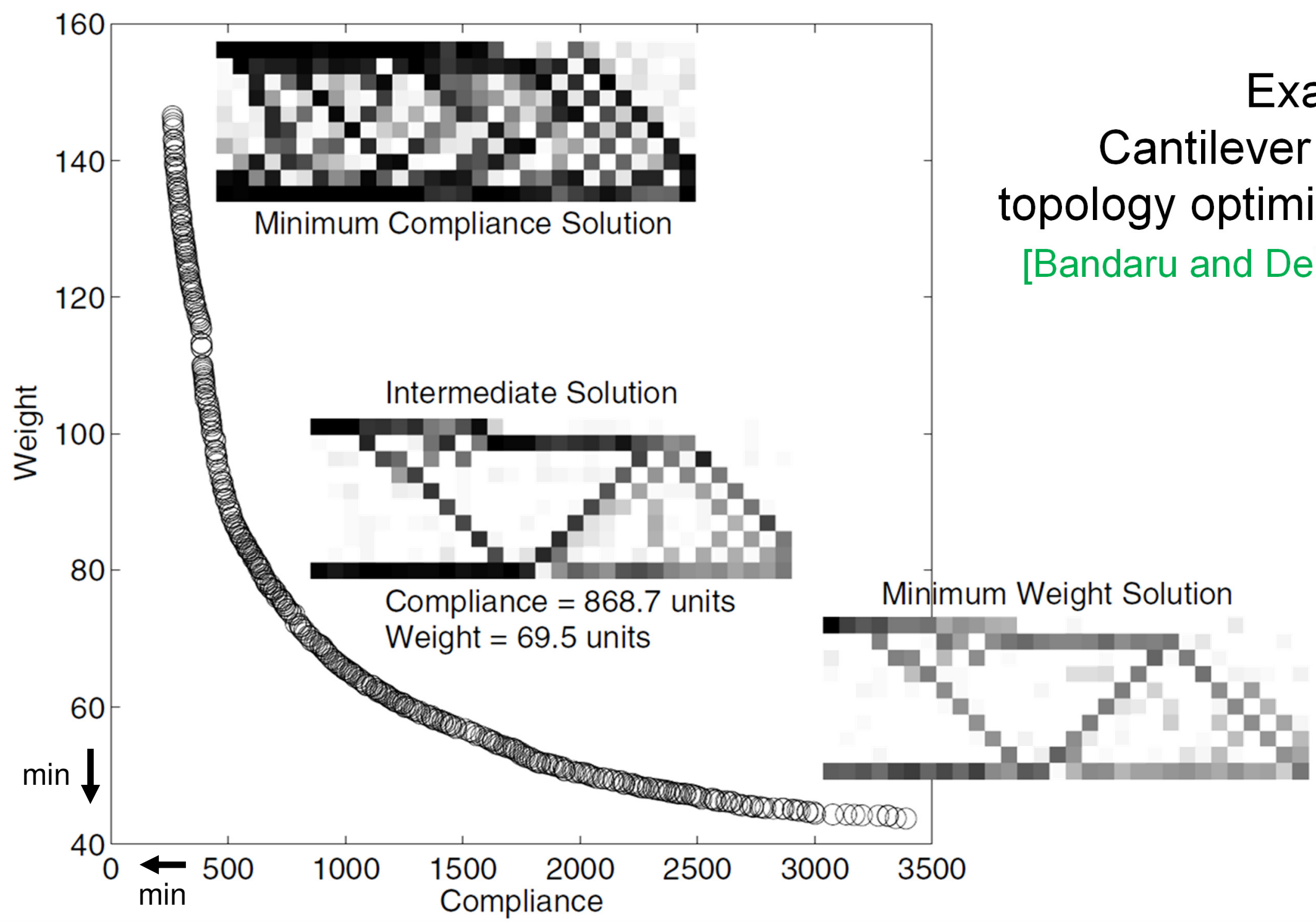




\section{Innovization}

Often innovative design principles among solutions are found

Example: Clutch brake design

[Deb and Srinivasan 2006]

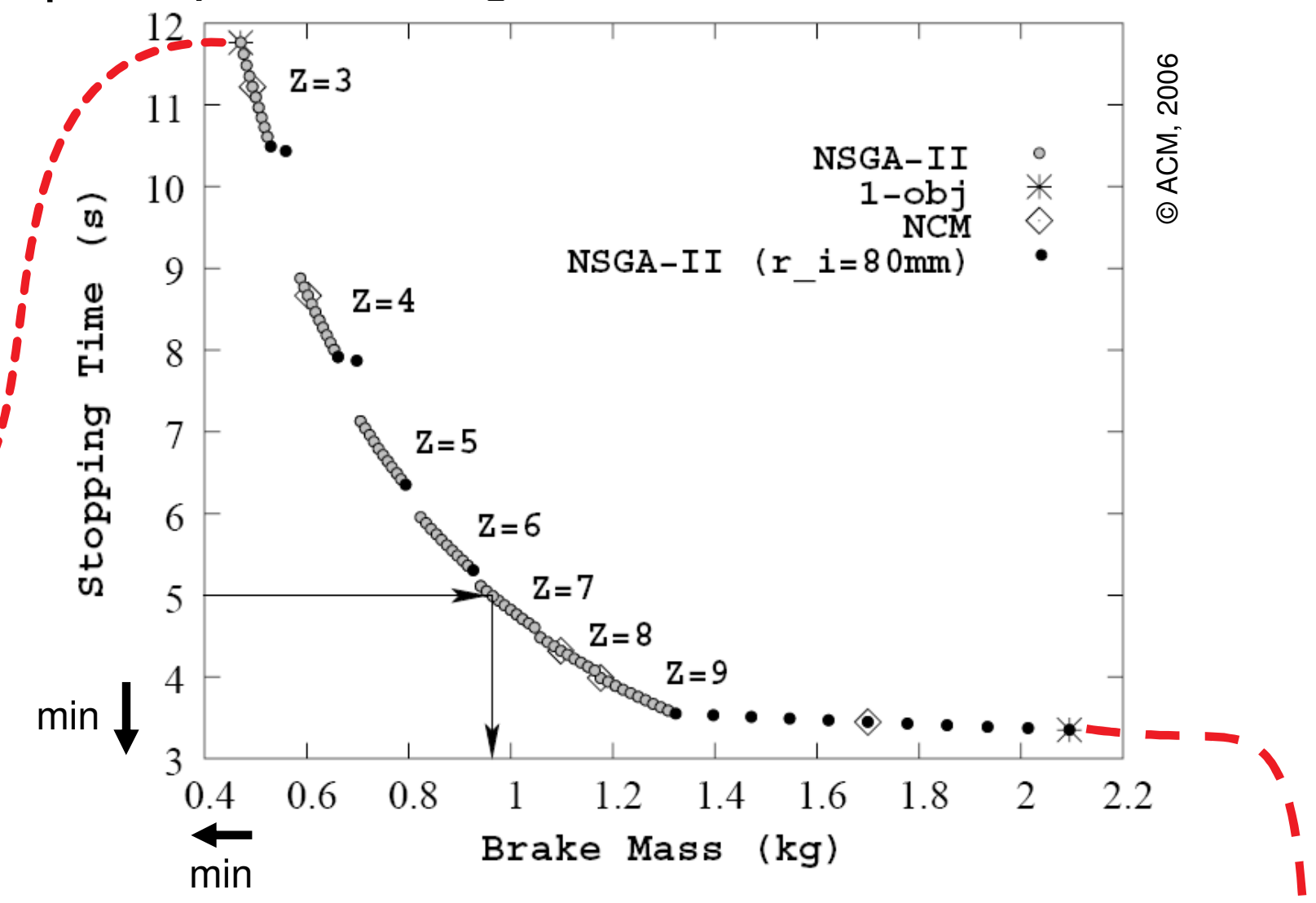




\section{Innovization}

Often innovative design principles among solutions are found

\section{Innovization [Deb and Srinivasan 2006]}

= using machine learning techniques to find new and innovative design principles among solution sets

= learning from/about a multi-objective optimization problem

\section{Other examples:}

- SOM for supersonic wing design [Obayashi and Sasaki 2003]

- Biclustering for processor design and knapsack [Ulrich et al. 2007]

- Successful case studies in engineering (noise barrier design, polymer extrusion, friction stir welding) [Deb et al. 2014] 


\section{The History of EMO At A Glance}

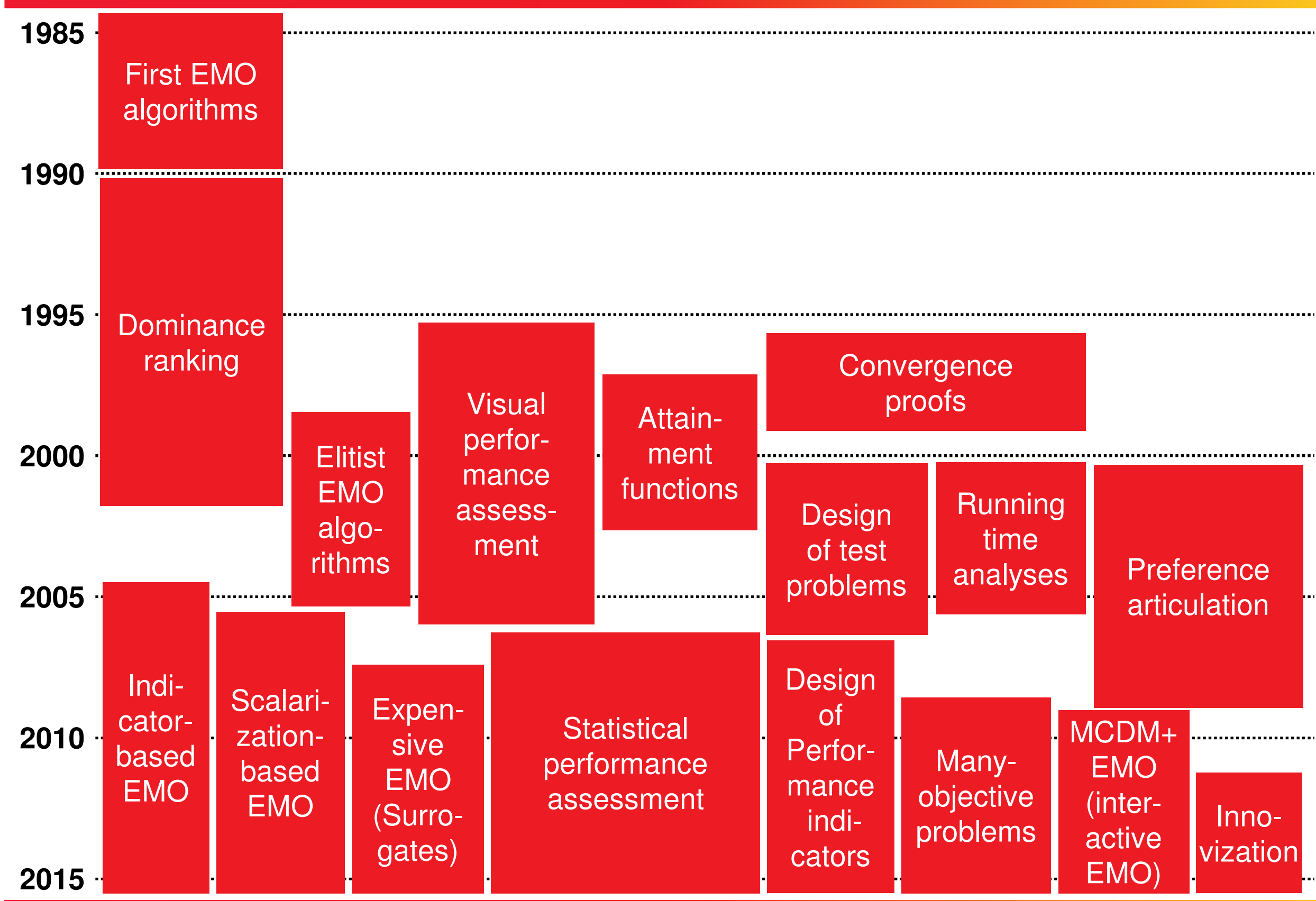




\section{The History of EMO At A Glance}

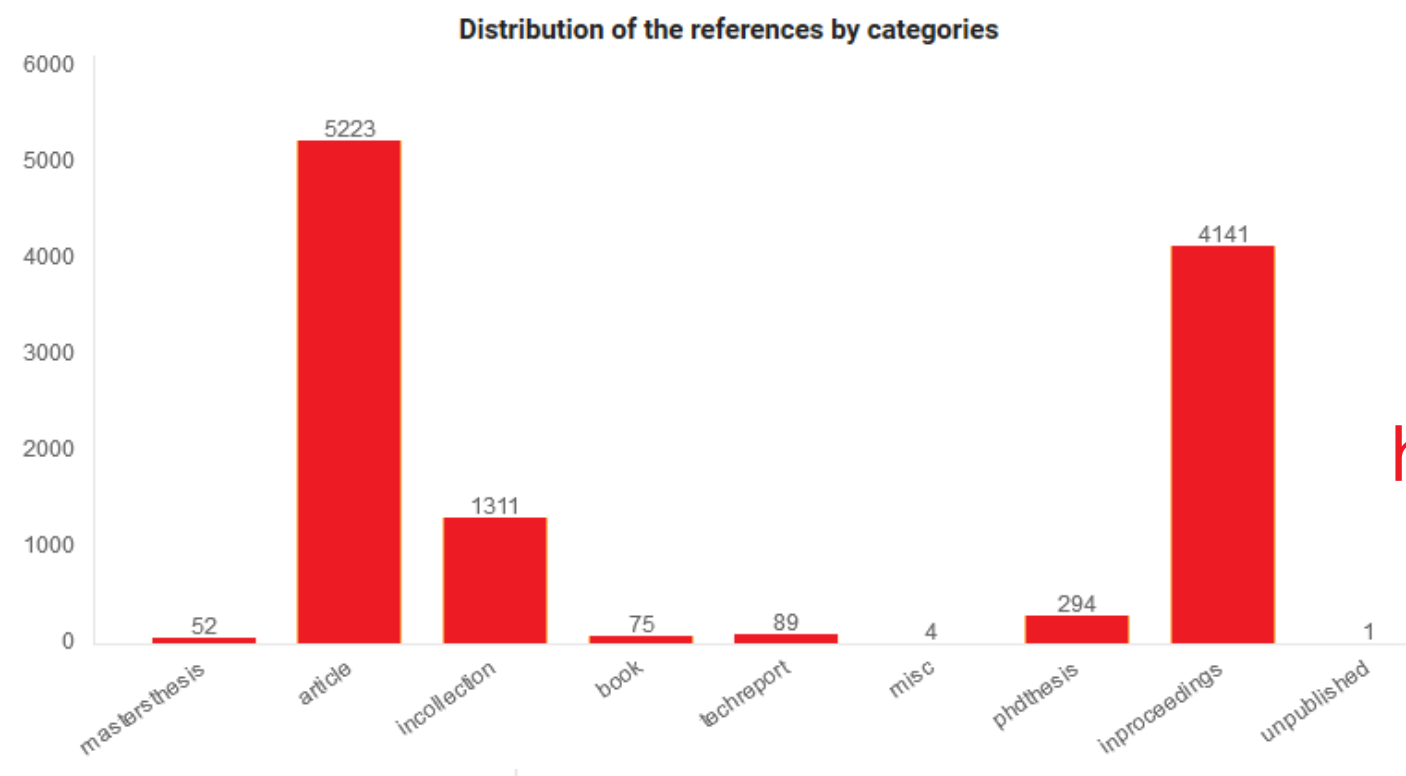

https://emoo.cs.cinvestav.mx/

Overall: 11190 references by March 26, 2018

800

600

400

200 


\section{The EMO Community}

\section{The EMO conference series:}

EMO 2019

East Lansing, MI, USA

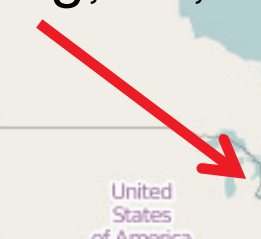

EMO 2015 Guimarães, PT

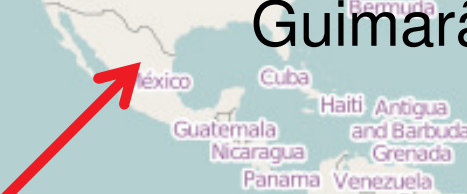

EMO 2005

\section{EMO 2003}

Faro, PT

EMO 2013 Sheffield, GB

EMO 2017 Münster, DE

\section{EMO 2009} Nantes, FR
Guanajuato, MX

Many further activities:
Ouro Preto, BR

\section{EMO 2001}

Zurich, $\mathrm{CH}$

EMO 2011

special sessions, special journal issues, workshops, tutorials, ... 


\title{
Overview
}

The Big Picture

Basic Algorithm Design Principles and Concepts

Performance Assessment and Benchmarking

\author{
Preference Articulation
}




\section{Fitness Assignment: Principal Approaches}

aggregation-based

problem decomposition

(multiple single-objective

optimization problems)

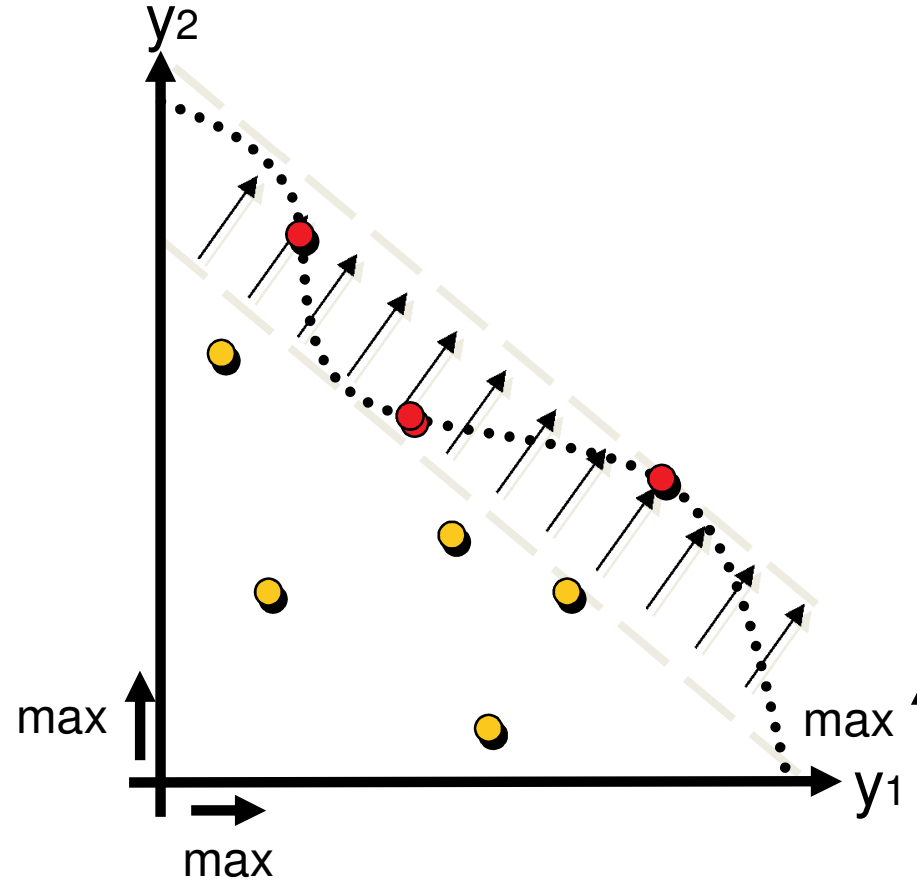

$\max$

solution-oriented scaling-dependent criterion-based

VEGA
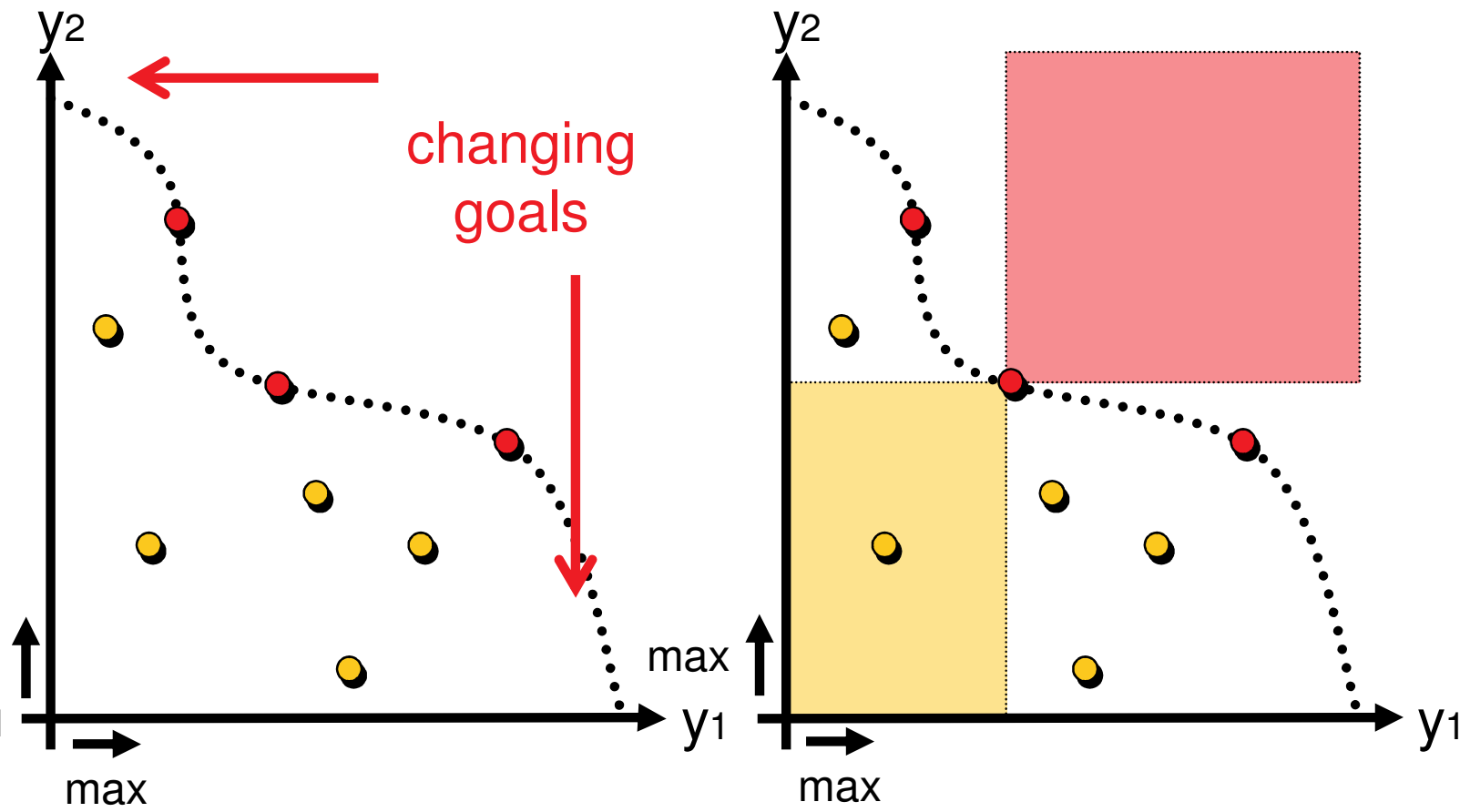

set-oriented scaling-independent 


\section{Solution-Oriented Problem Transformations}

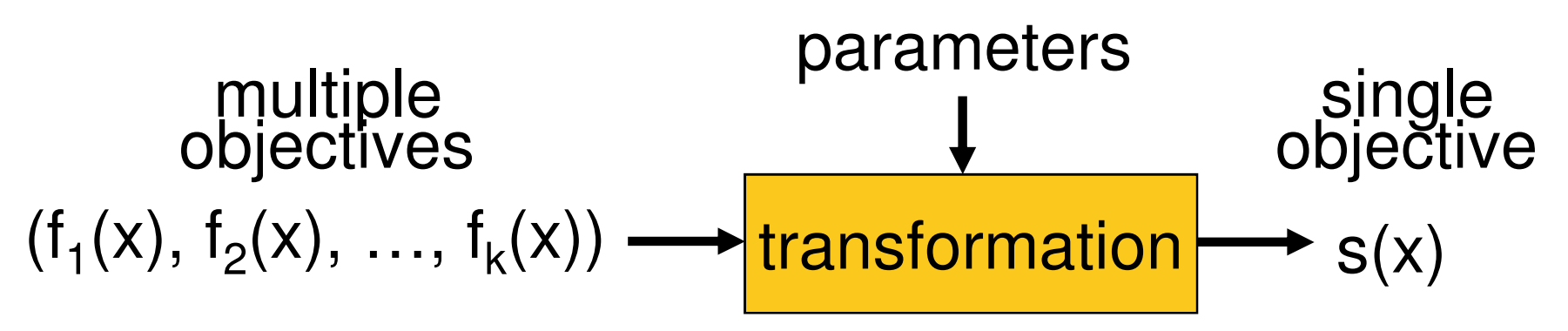

A scalarizing function $s$ is a function $s: Z \rightarrow \mathbb{R}$ that maps each objective vector $u=\left(u_{1}, \ldots, u_{n}\right) \in Z$ to a real value $s(u) \in \mathbb{R}$ 


\section{Solution-Oriented Problem Transformations}
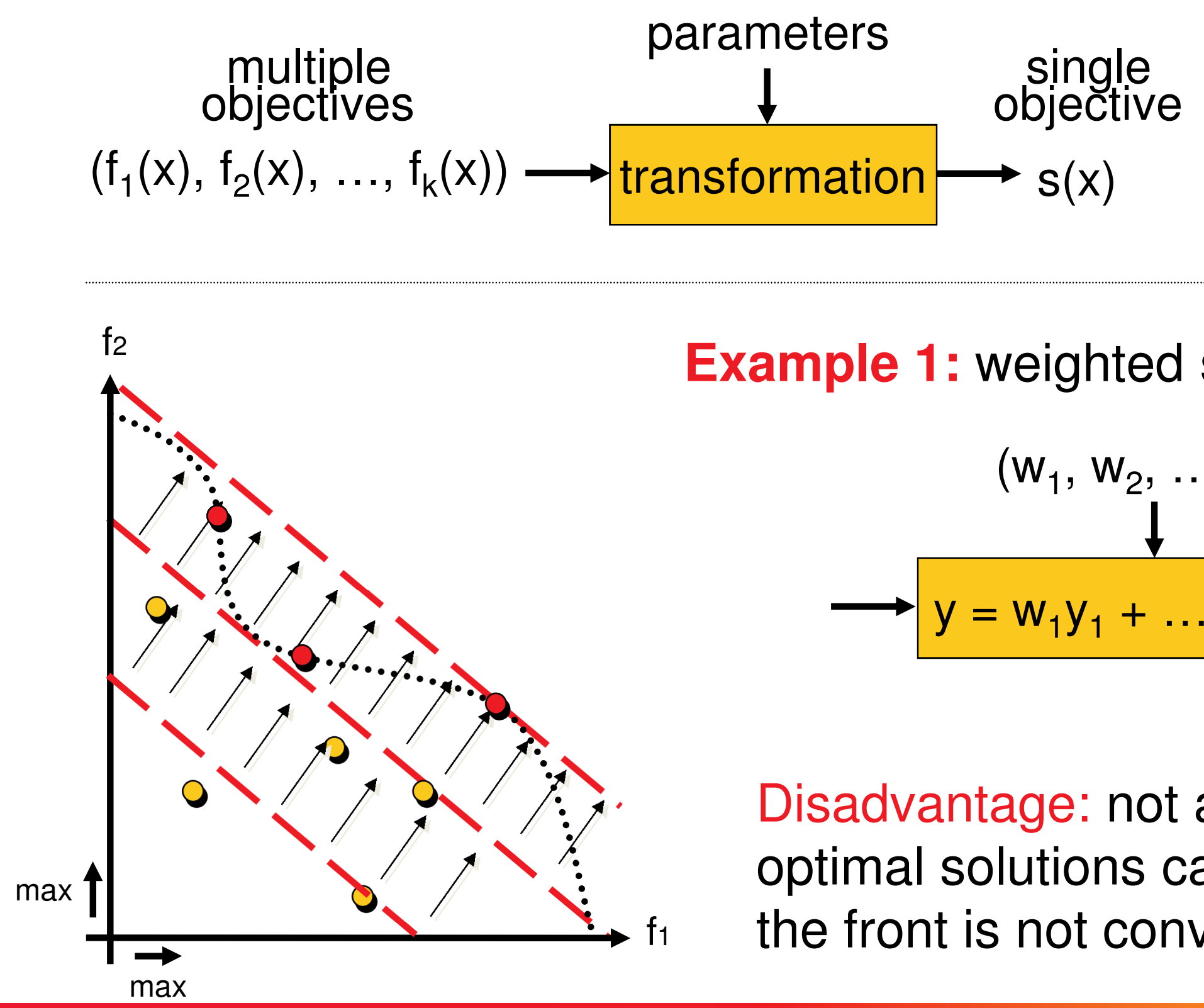

Example 1: weighted sum approach

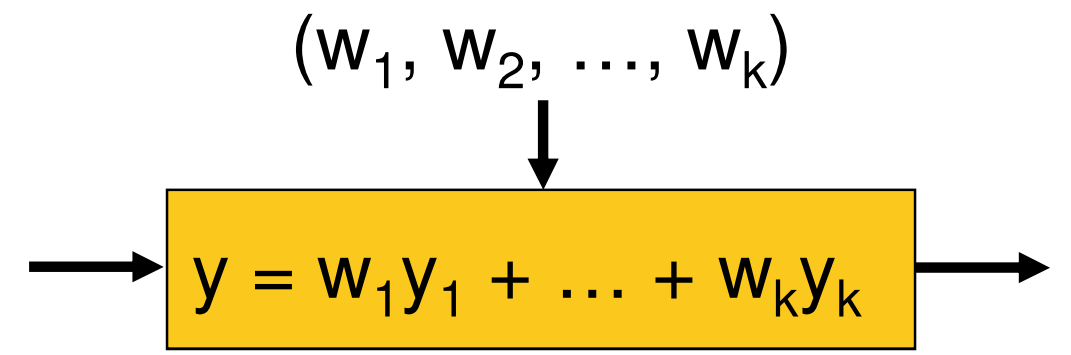

Disadvantage: not all Paretooptimal solutions can be found if the front is not convex 


\section{Solution-Oriented Problem Transformations}
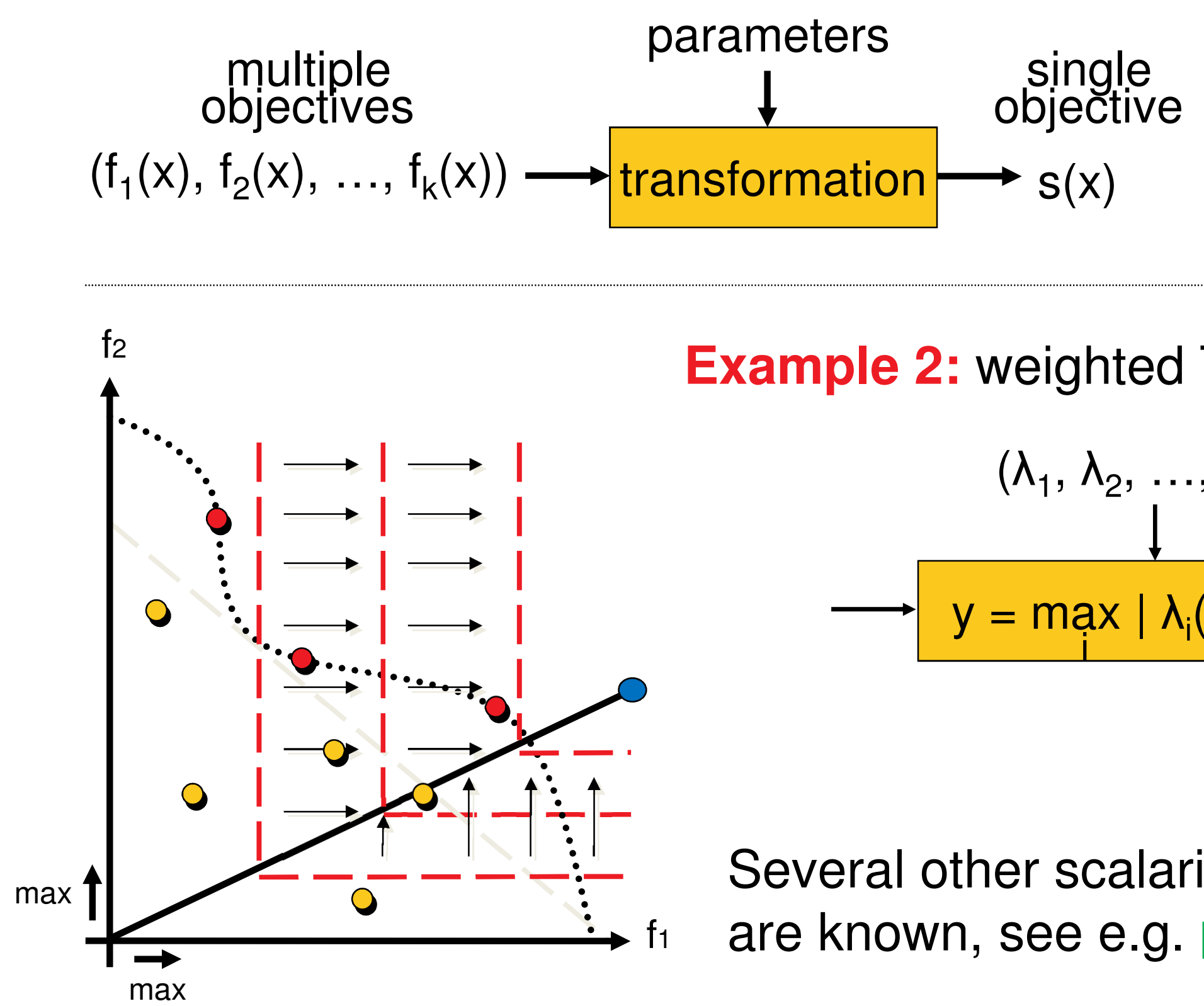

Example 2: weighted Tchebycheff

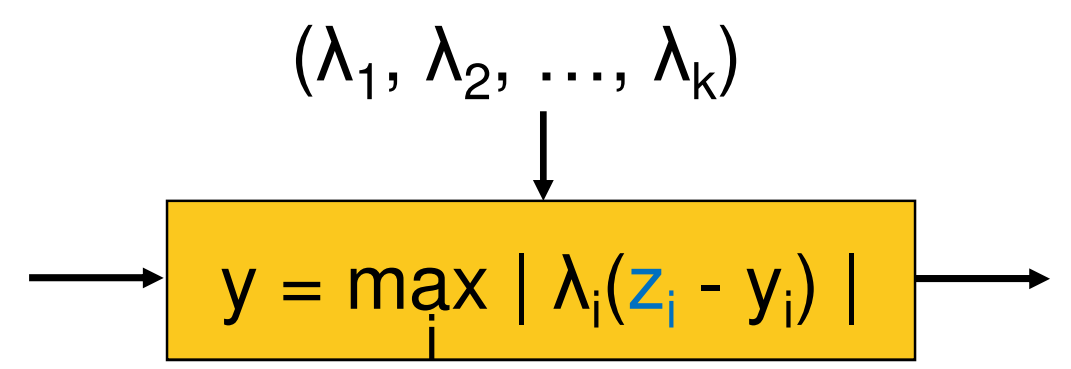

Several other scalarizing functions are known, see e.g. [Miettinen 1999] 


\section{General Scheme of Most Set-Oriented EMO}

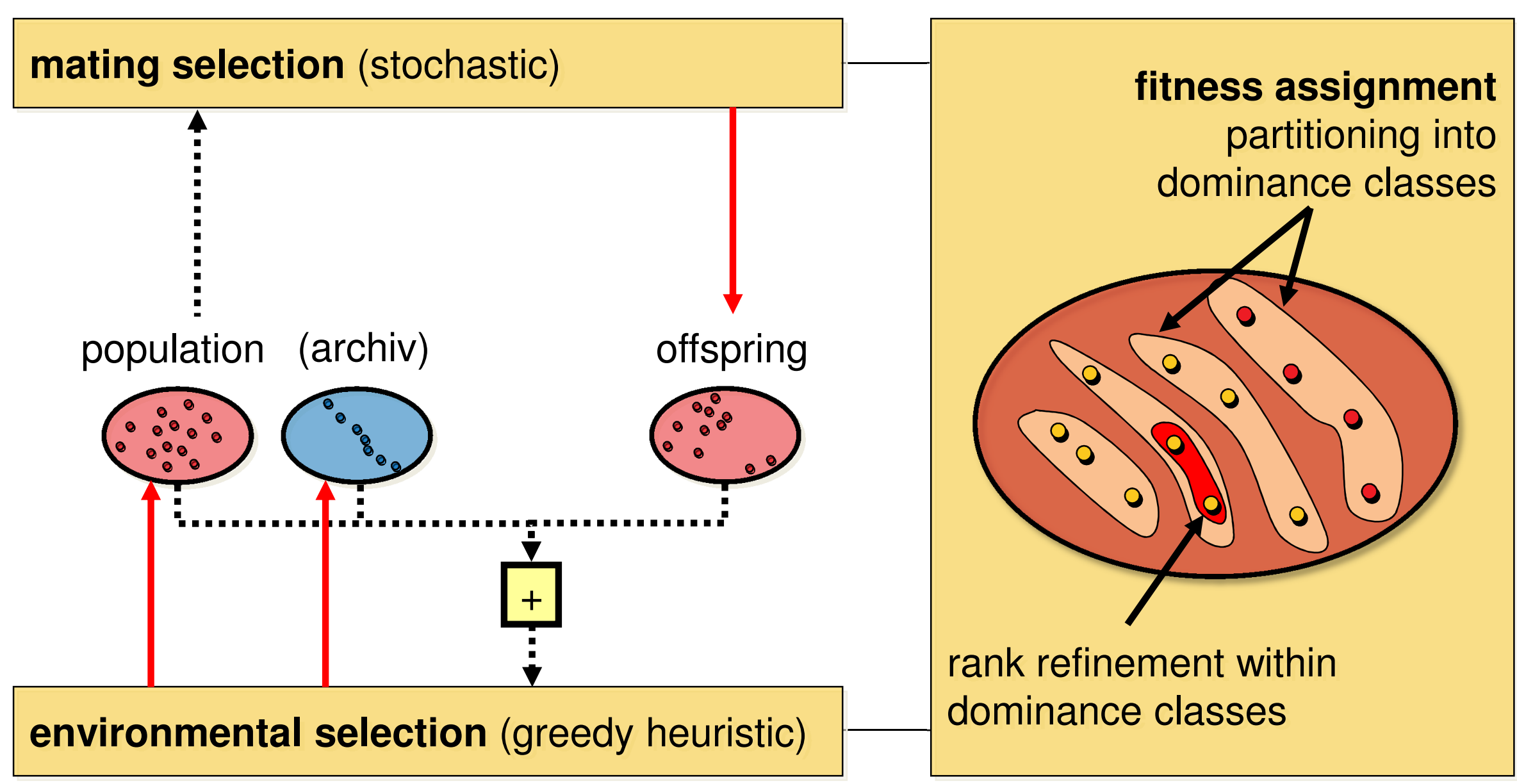




\section{Ranking of the Population Using Dominance}

... goes back to a proposal by David Goldberg in 1989.

... is based on pairwise comparisons of the individuals only.

- dominance rank: by how many individuals is an individual dominated? MOGA, NPGA

- dominance count: how many individuals does an individual dominate?

SPEA, SPEA2

- dominance depth: at which front is an individual located? NSGA, NSGA-II, most of the recently proposed algorithms

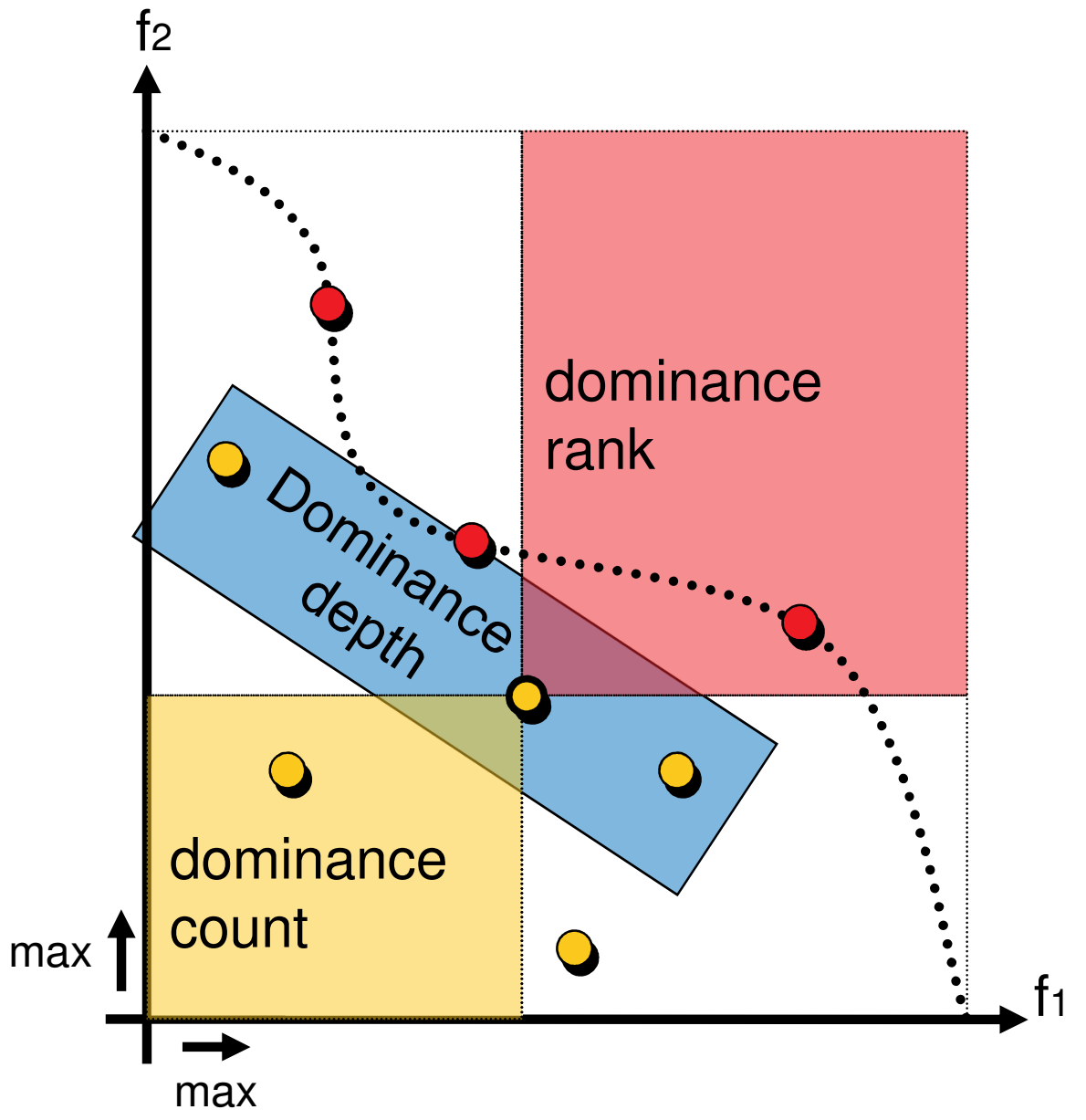




\section{Illustration of Dominance-Based Partitioning}

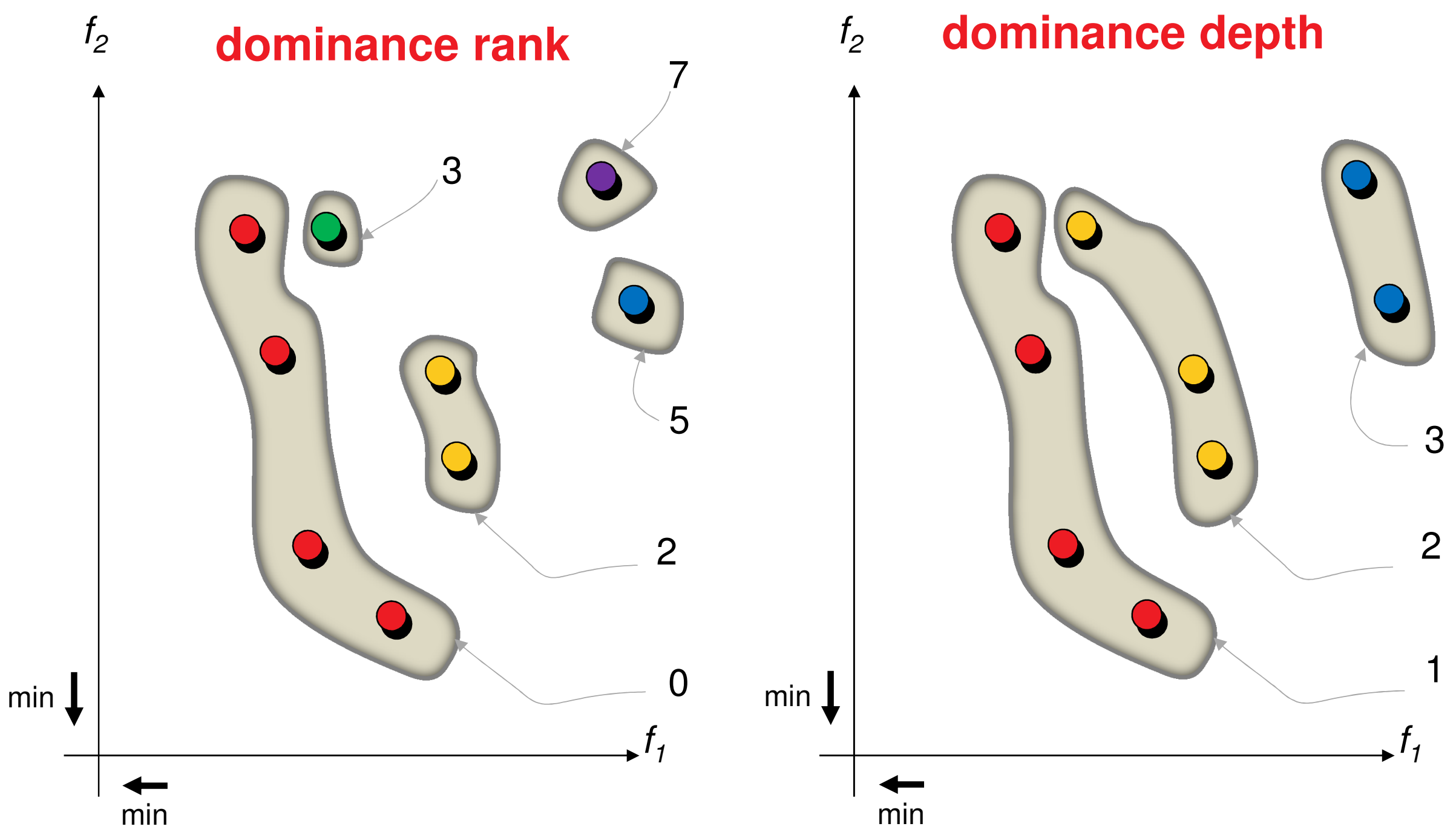




\section{Refinement of Dominance Rankings}

Goal: rank incomparable solutions within a dominance class

( Diversity information

Kernel method

diversity $=$ function of the distances

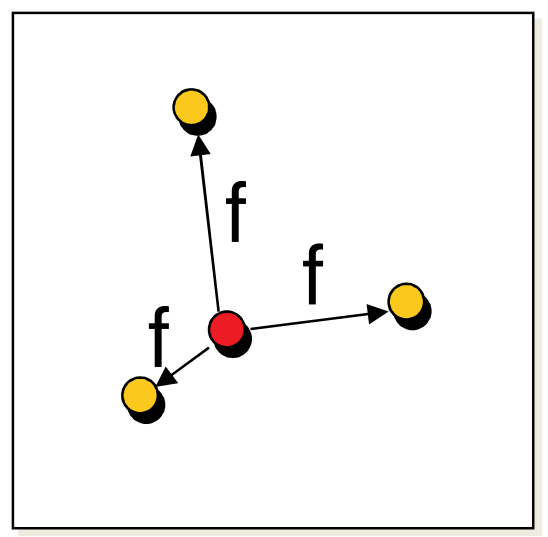

k-th nearest neighbor

diversity $=$ function of distance to $k$-th nearest neighbor

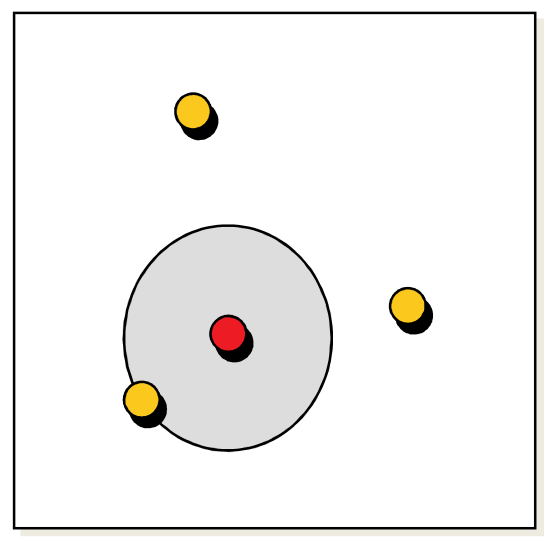

Histogram method diversity $=$ number of elements within box(es)

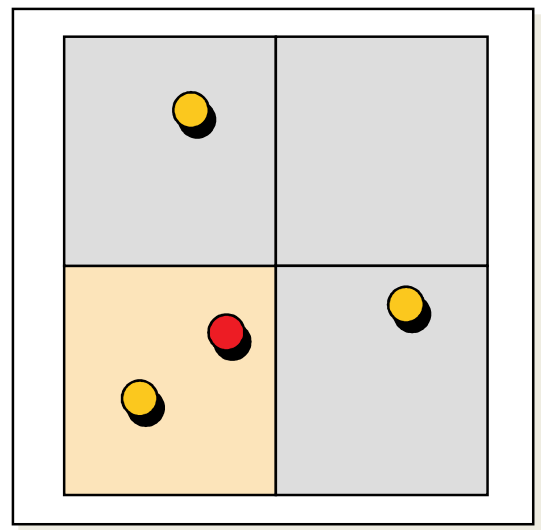

2 (Contribution to a) quality indicator 


\section{Example: NSGA-II Diversity Preservation}

\section{Crowding Distance (CD)}

- sort solutions with regard to each objective

- assign CD maximum value to extremal objective vectors

- compute CD based on the distance to the neighbors in each objective

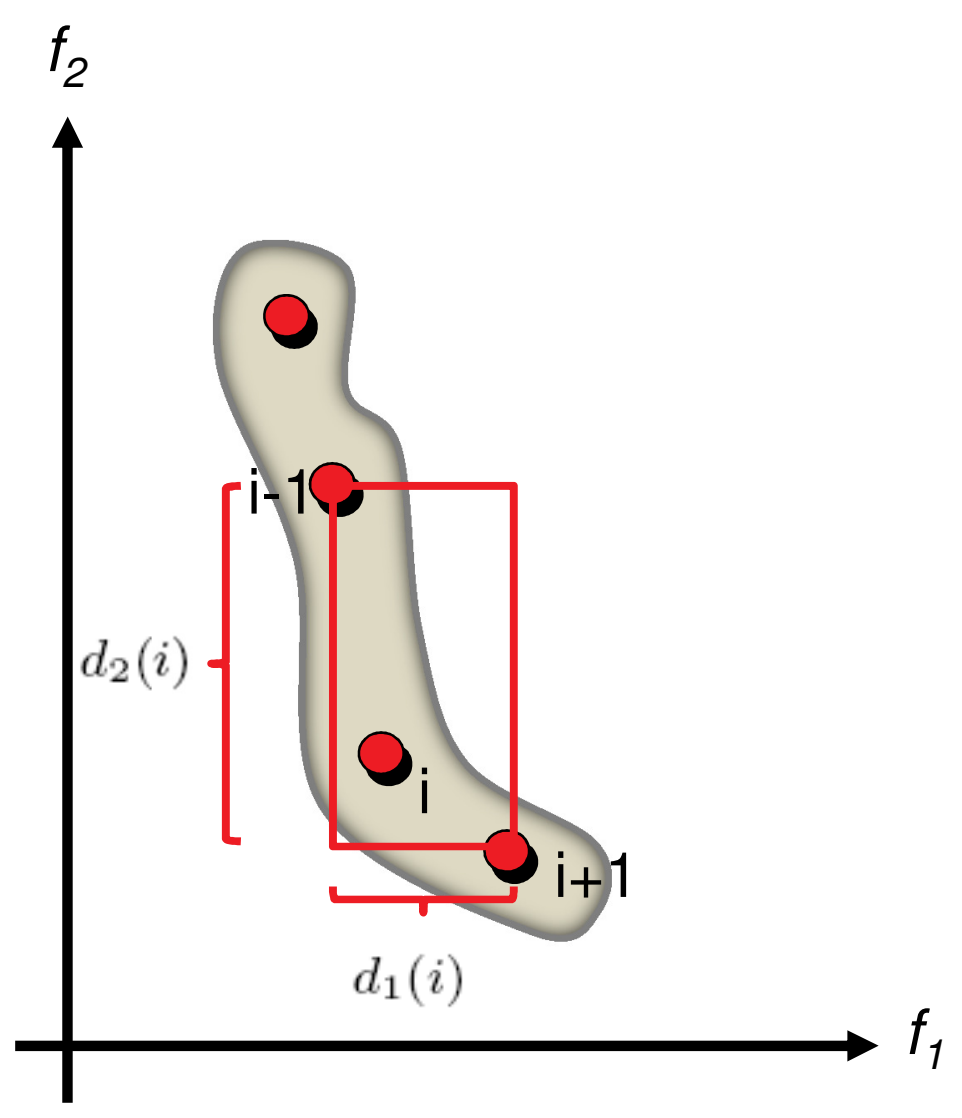

$$
\mathrm{CD}(i)=\frac{d_{1}(i)}{f_{1, \max }-f_{1, \min }}+\cdots+\frac{d_{m}(i)}{f_{m, \text { max }}-f_{m, \text { min }}}
$$




\section{SPEA2 and NSGA-II: Deteriorative Cycles}

Selection in SPEA2 and NSGA-II can result in deteriorative cycles

non-dominated solutions already found can be lost

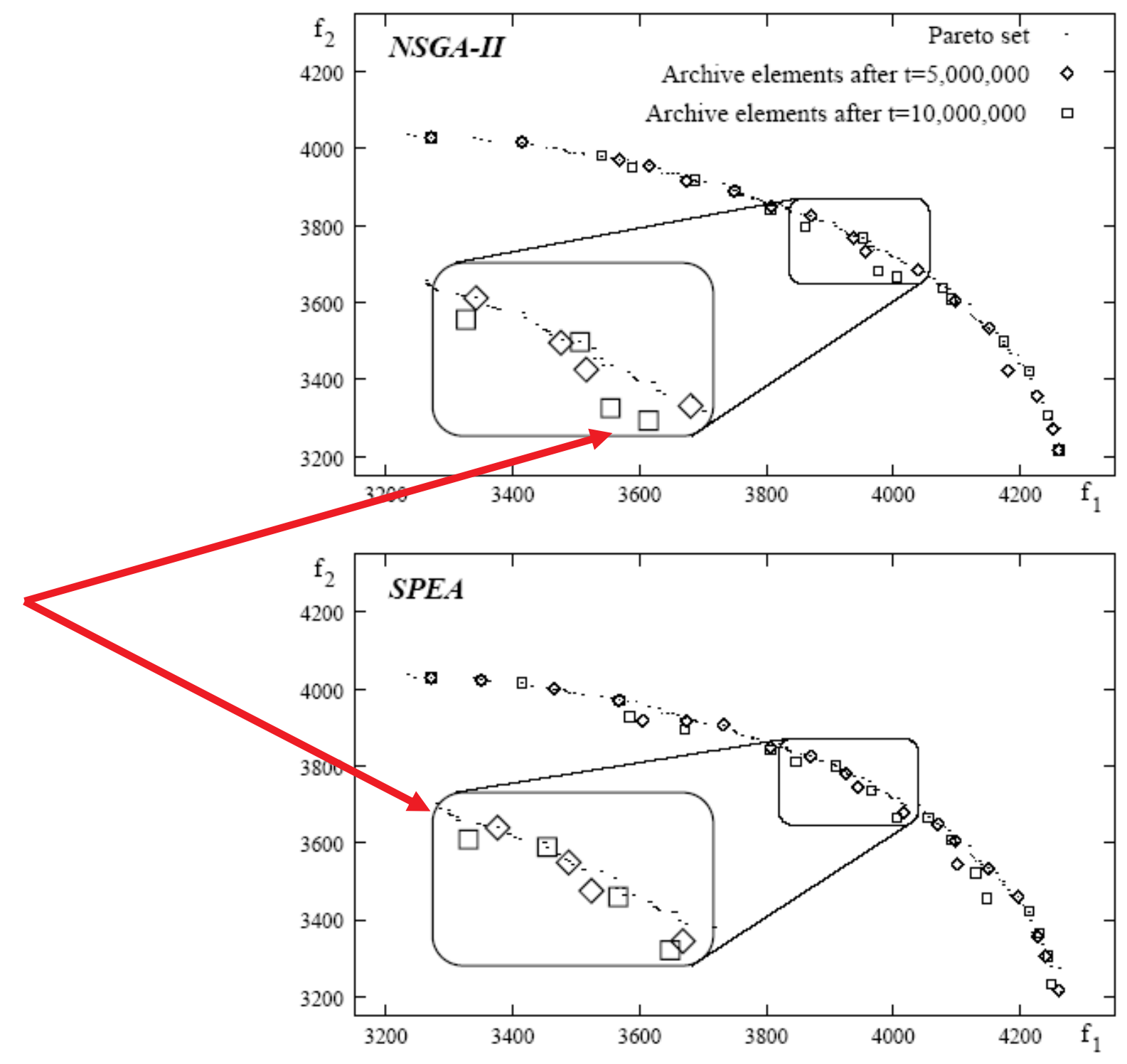




\section{Remark: Many-Objective Optimization}

- high number of objectives

$\rightarrow$ percentage of non-dominated solutions within a random sample quickly approaches $100 \%$

$\rightarrow$ optimization is mainly guided by diversity criterion

$\rightarrow$ apply secondary criterion compliant with dominance relation
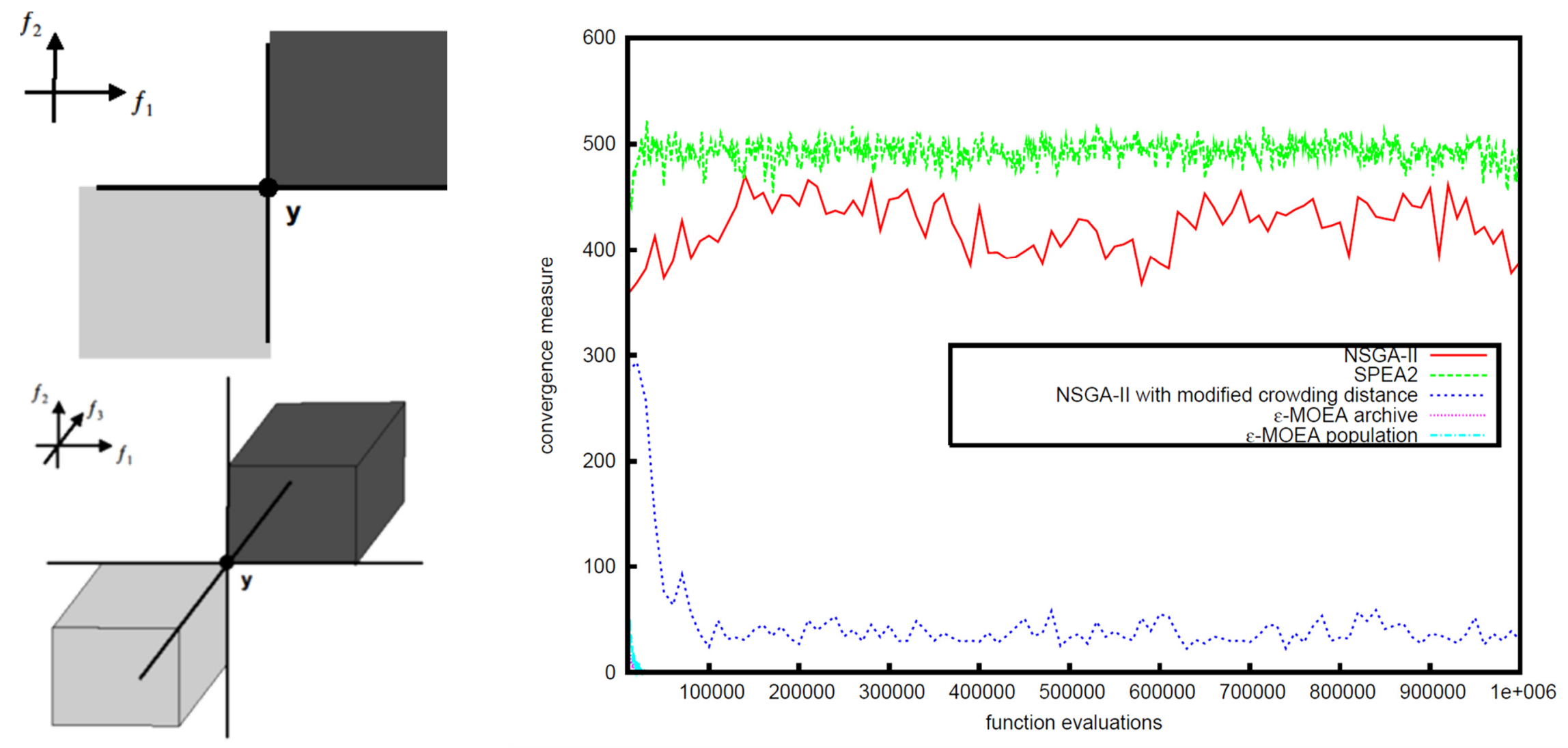


\section{Hypervolume-Based Selection}

Latest Approach (SMS-EMOA, MO-CMA-ES, HypE, ...)

use hypervolume indicator to guide the search: refines dominance

\section{Main idea}

Delete solutions with the smallest

hypervolume contribution

$d(s)=I_{H}(P)-I_{H}(P /\{s\})$ iteratively

\section{But:}

- can also result in cycles if reference

point is not constant [Judt et al. 2011]

- expensive to compute exactly [Bringmann and Friedrich 2009]

- less and less practically important [Guerreiro and Fonseca 2017] 


\section{Indicator-Based Selection}

- Concept can be generalized to any quality indicator
A (unary) quality indicator $I$ is a function $I: \Psi=2^{X} \mapsto \mathbb{R}$ that assigns a Pareto set approximation a real value.

Multiobjective Problem

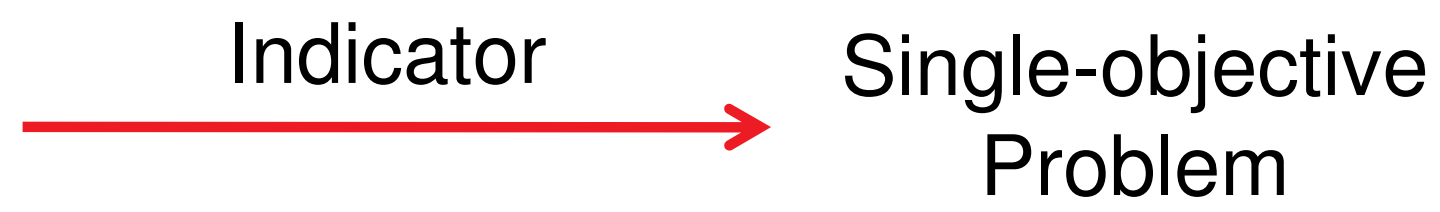

- for example: R2-indicator [Brockhoff et al. 2012], [Trautmann et al. 2013], [Díaz-Manríquez et al. 2013]

- Generalizable also to contribution to larger sets

HypE [Bader and Zitzler 2011]: Hypervolume sampling + contribution if more than 1 (random) solution deleted 


\section{The Optimization Goal in Indicator-Based EMO}

When the goal is to maximize a unary indicator...

- we have a single-objective problem on sets

- but what is the optimum?

- important: population size $\mu$ plays a role!

\section{Optimal $\mu$-Distribution:}

A set of $\mu$ solutions that maximizes a certain unary indicator I among all sets of $\mu$ solutions is called optimal $\mu$-distribution for I. [Auger et al. 2009a]

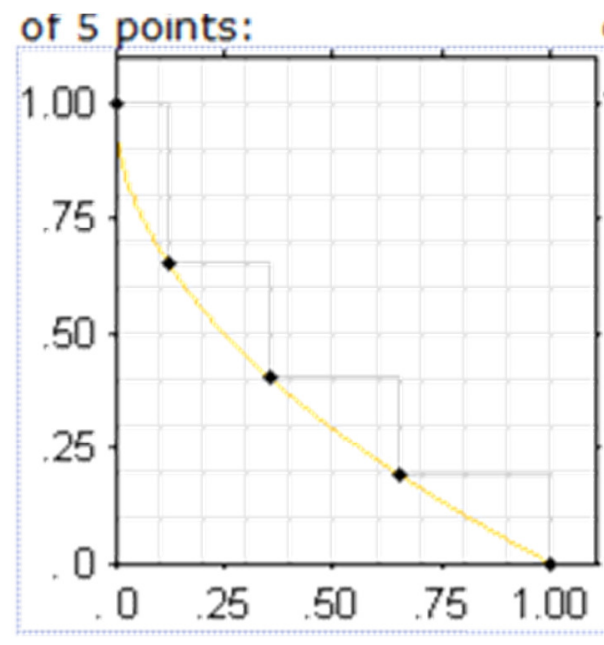

of 10 points:

of 20 points:

of 50 points:

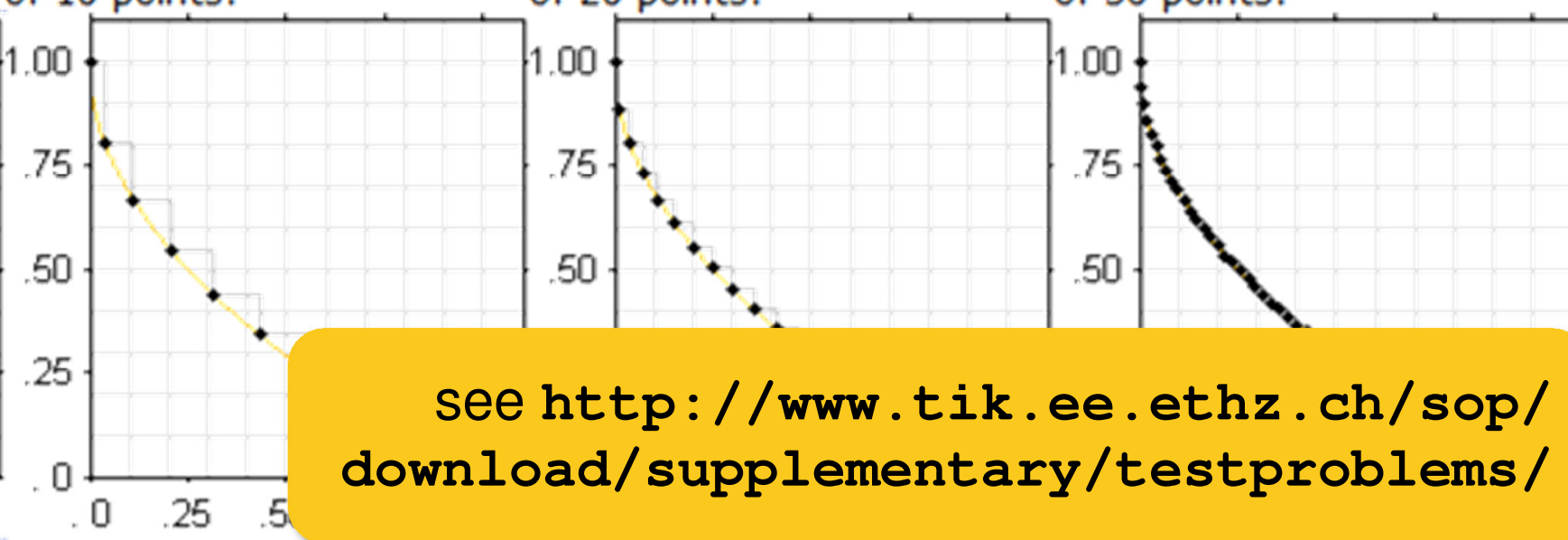




\section{Optimal $\mu$-Distributions for the Hypervolume}

Hypervolume indicator refines dominance relation

$\Rightarrow$ most results on optimal $\mu$-distributions for hypervolume

\section{Optimal $\mu$-Distributions (example results)}

[Auger et al. 2009a]:

- contain equally spaced points iff front is linear

- density of points $\propto \sqrt{-f^{\prime}(x)}$ with $f^{\prime}$ the slope of the front

[Friedrich et al. 2011]:

optimal $\mu$-distributions for the hypervolume correspond to $\varepsilon$-approximations of the front

$$
\begin{array}{lr}
\text { OPT } & 1+\frac{\log (\min \{A / a, B / b\})}{n} \\
\text { HYP } & 1+\frac{\sqrt{A / a}+\sqrt{B / b}}{n-4} \\
\text { logHYP } & 1+\frac{\sqrt{\log (A / a) \log (B / b)}}{n-2}
\end{array}
$$

! (probably) does not hold for $>2$ objectives 


\section{Indicator-Based EMO}

\section{Open Questions:}

- How do the optimal $\mu$-distributions look like for $>2$ objectives?

- how to compute certain indicators quickly in practice?

- several recent improvements for the hypervolume indicator

[Yildiz and Suri 2012], [Bringmann 2012], [Bringmann 2013]

[Guerreiro and Fonseca 2017]

- how to do indicator-based subset selection quickly?

- also here several recent improvements

[Kuhn et al. 2014], [Bringmann et al. 2014], [Guerreiro et al. 2015]

- what is the best strategy for the subset selection?

further open questions on indicator-based EMO available at http://simco.gforge.inria.fr/doku.php?id=openproblems 


\section{Decomposition-Based Selection: MOEA/D}

MOEA/D: Multiobjective Evolutionary Algorithm Based on Decomposition [Zhang and Li 2007]

\section{Ideas:}

- optimize $\mathrm{N}$ scalarizing functions in parallel

- use best solutions of neighbor subproblems for mating

- keep the best solution for each scalarizing function

- update neighbors

- use external archive for non-dominated solutions

- several variants and enhancements

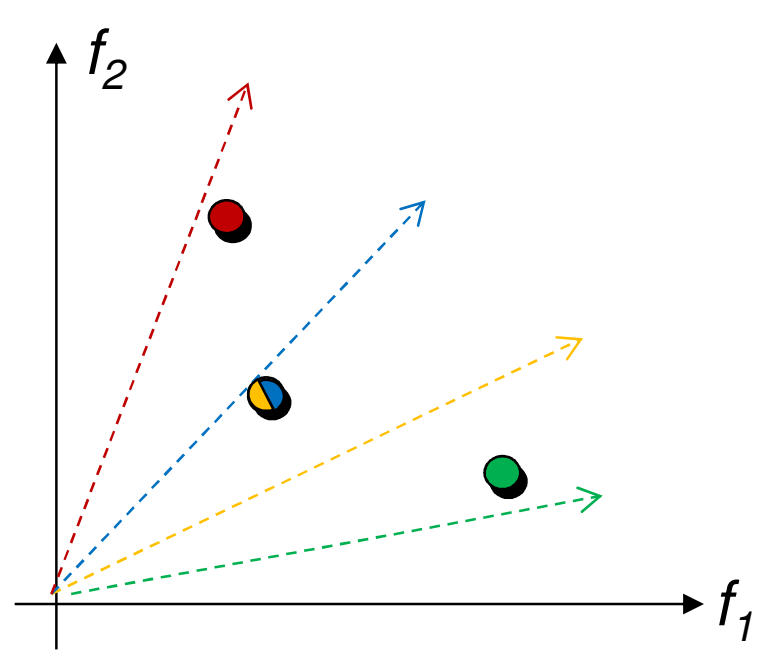




\section{Remark: Variation in EMO}

- at first sight not different from single-objective optimization

- most research on selection mechanisms (until now)

- but: convergence to a set $\neq$ convergence to a point

\section{Open Question:}

- how to achieve fast convergence to a set?

\section{Related work:}

- set-based gradient of the HV [Emmerich et al. 2007]

- multiobjective CMA-ES [lgel et al. 2007, Voß et al. 2010, Krause et al. 2016]

- RM-MEDA [Zhang et al. 2008]

- set-based variation [Bader et al. 2009]

- set-based fitness landscapes [Verel et al. 2011]

- offline and online configuration based on libraries of variation operators [Bezerra et al. 2015, Hadka and Reed 2013] 


\section{Overview}

The Big Picture

Basic Algorithm Design Principles and Concepts

Performance Assessment and Benchmarking

Preference Articulation 


\section{Once Upon a Time...}

... multiobjective EAs were mainly compared visually:

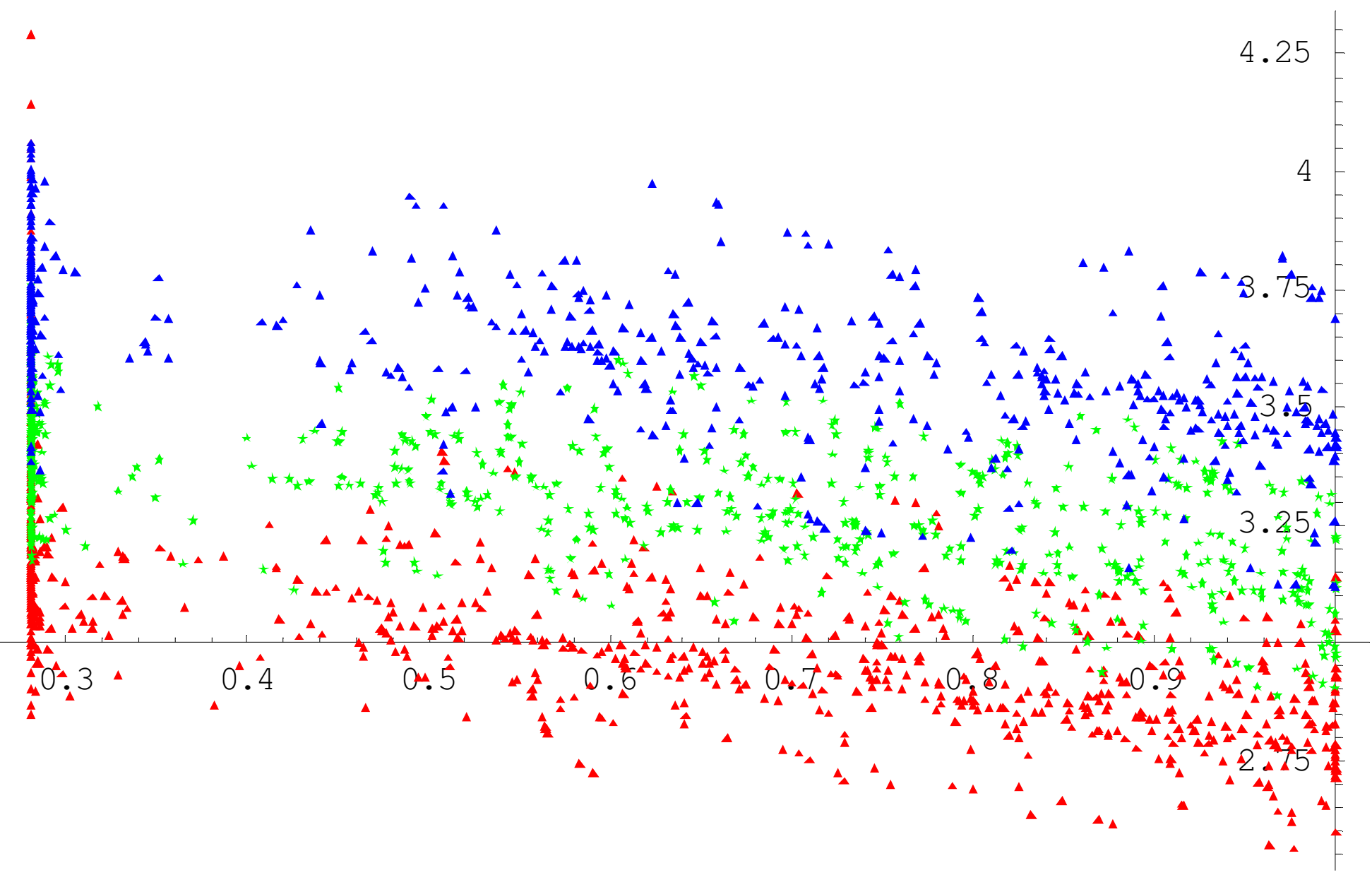

ZDT6 benchmark problem: IBEA, SPEA2, NSGA-II 


\section{Two Approaches for Empirical Studies}

\section{Attainment function approach}

- applies statistical tests directly to the approximation set

- detailed information about how and where performance differences occur

\section{Quality indicator approach}

- reduces each approximation set to a single quality value

- applies statistical tests to the quality values
$A$ attains

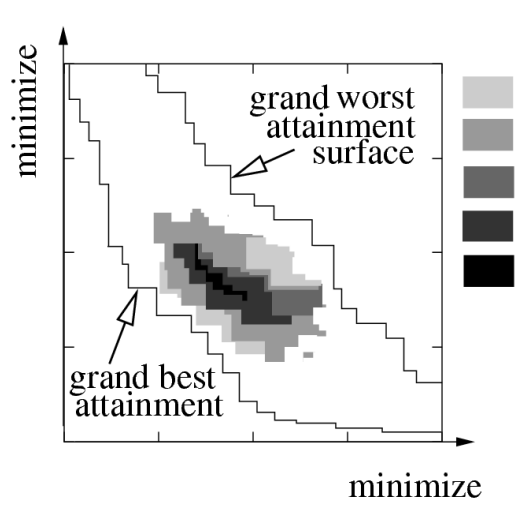

$B$ attains

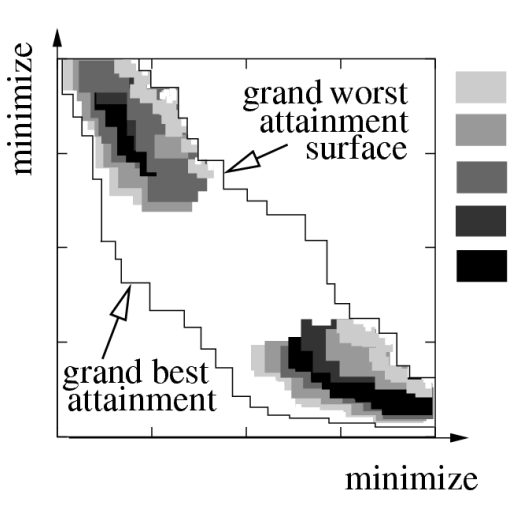

\begin{tabular}{r|cl}
\multicolumn{1}{c|}{ Indicator } & $\mathrm{A}$ & \multicolumn{1}{c}{$\mathrm{B}$} \\
\hline Hypervolume indicator & 6.3431 & 7.1924 \\
$\epsilon$-indicator & 1.2090 & 0.12722 \\
$R_{2}$ indicator & 0.2434 & 0.1643 \\
$R_{3}$ indicator & 0.6454 & 0.3475 \\
\hline
\end{tabular}

see e.g. [Zitzler et al. 2003] 


\section{Empirical Attainment Functions}

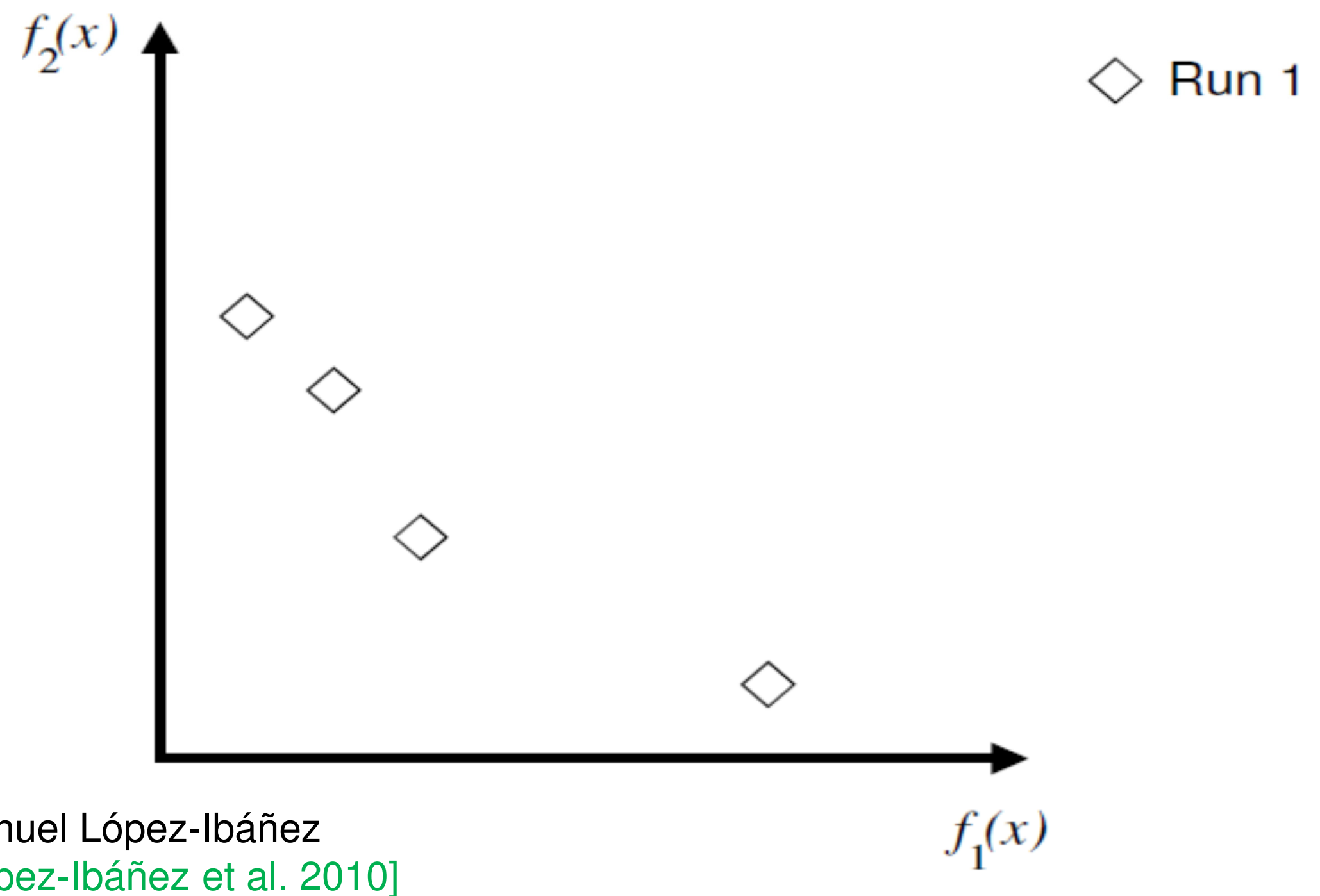

(c) Manuel López-lbáñez [López-lbáñez et al. 2010] 


\section{Empirical Attainment Functions}

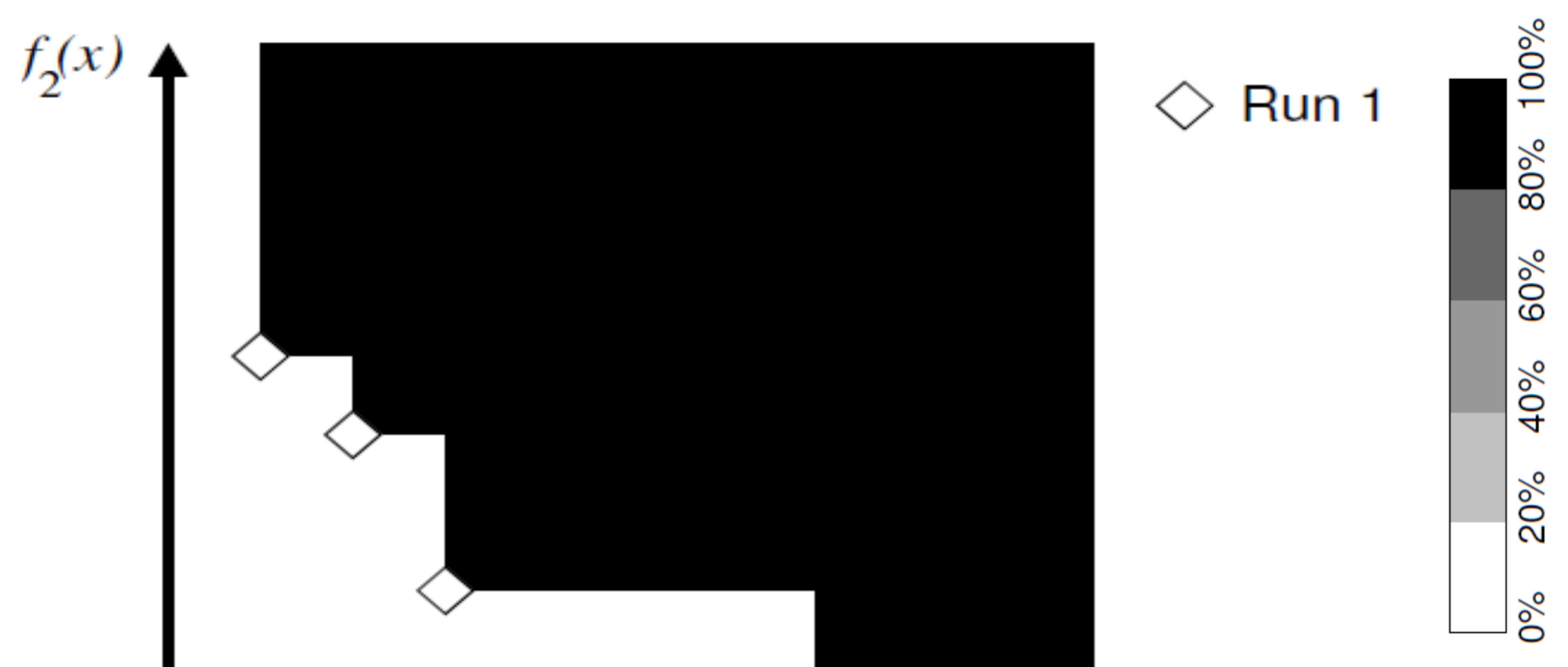

(C) Manuel López-lbáñez [López-lbáñez et al. 2010] 


\section{Empirical Attainment Functions}

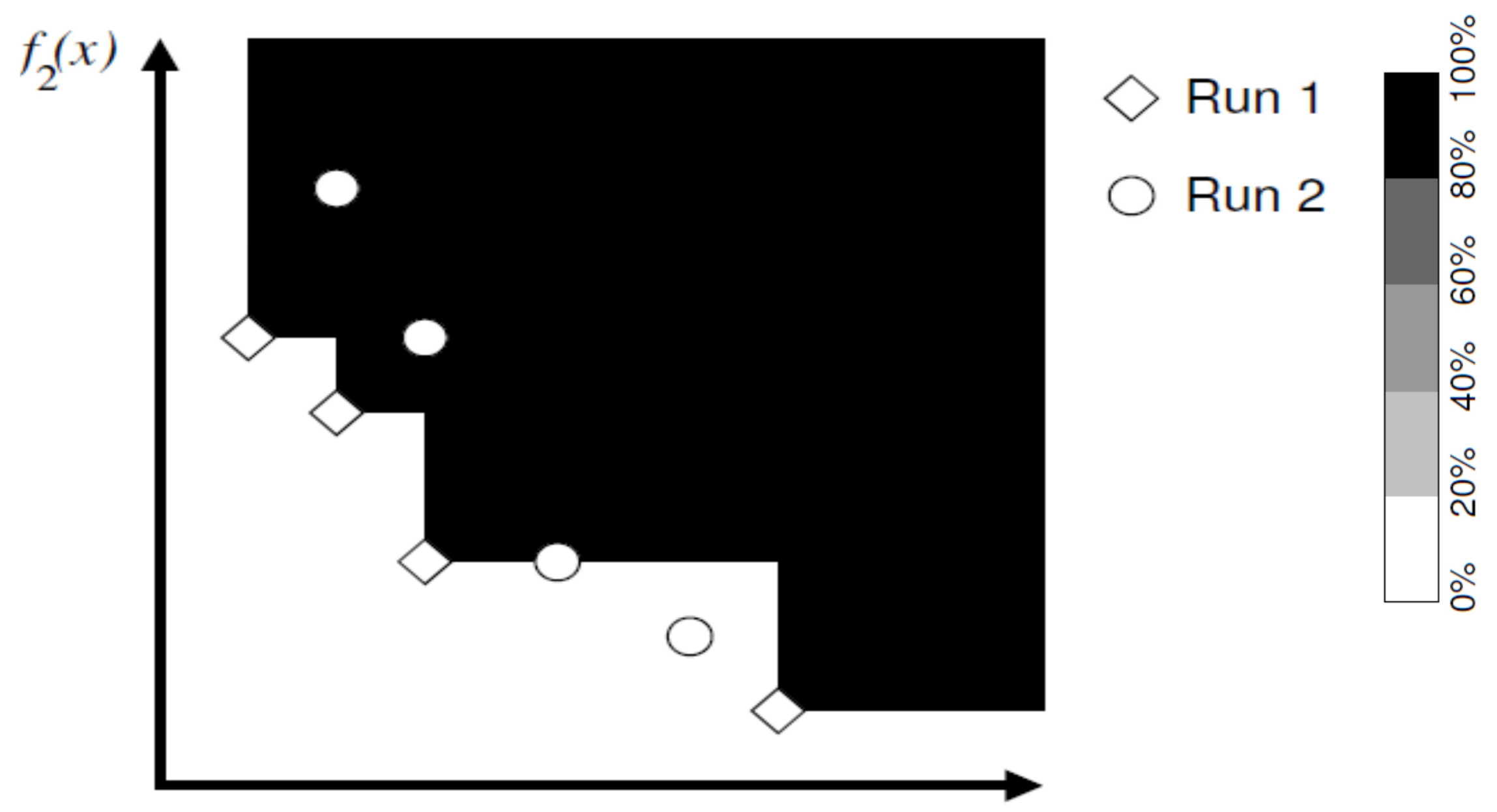

(C) Manuel López-lbáñez [López-lbáñez et al. 2010]

$$
f_{1}(x)
$$




\section{Empirical Attainment Functions}

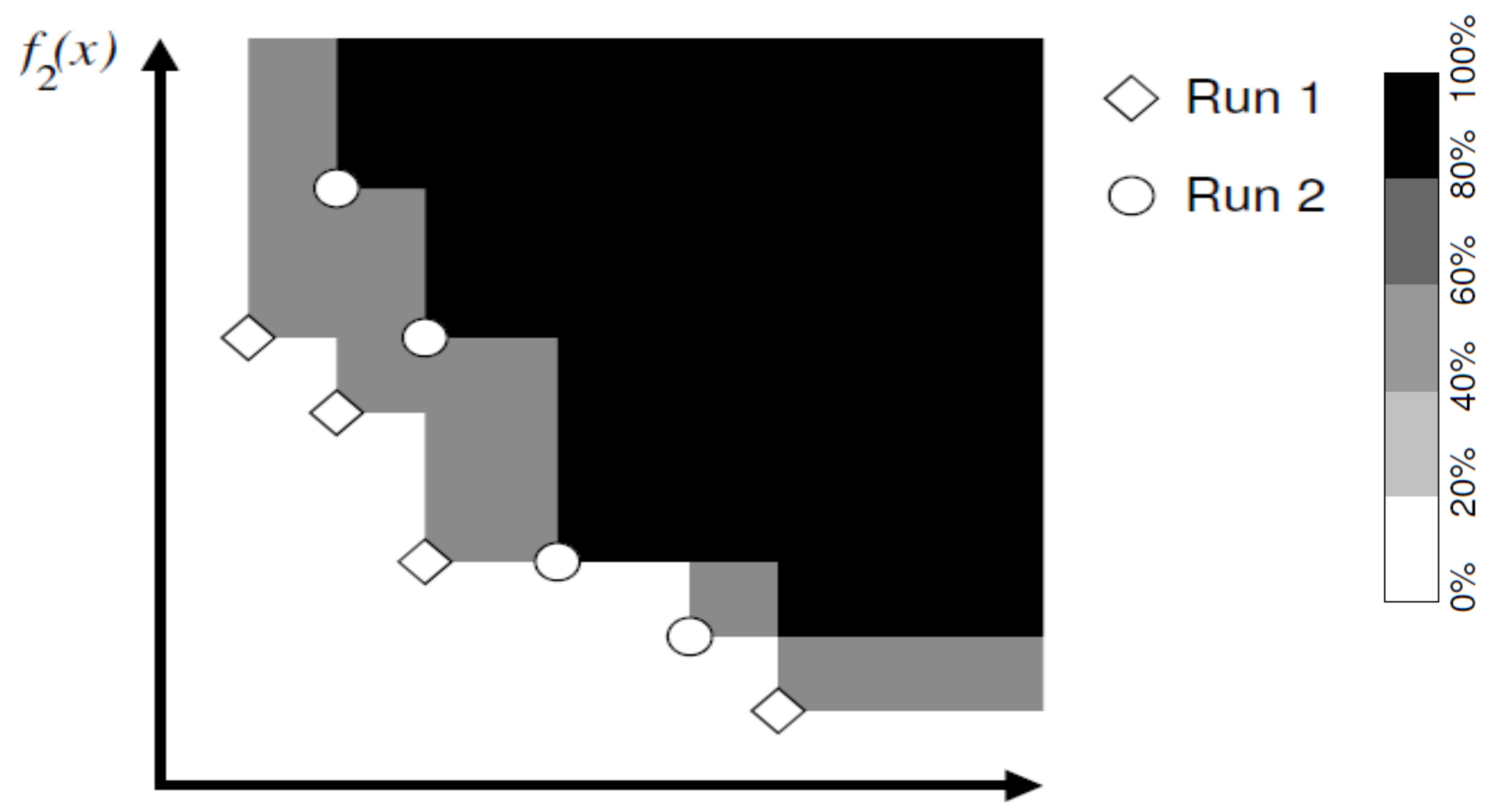

(C) Manuel López-lbáñez [López-lbáñez et al. 2010]

$$
f_{1}(x)
$$




\section{Empirical Attainment Functions}

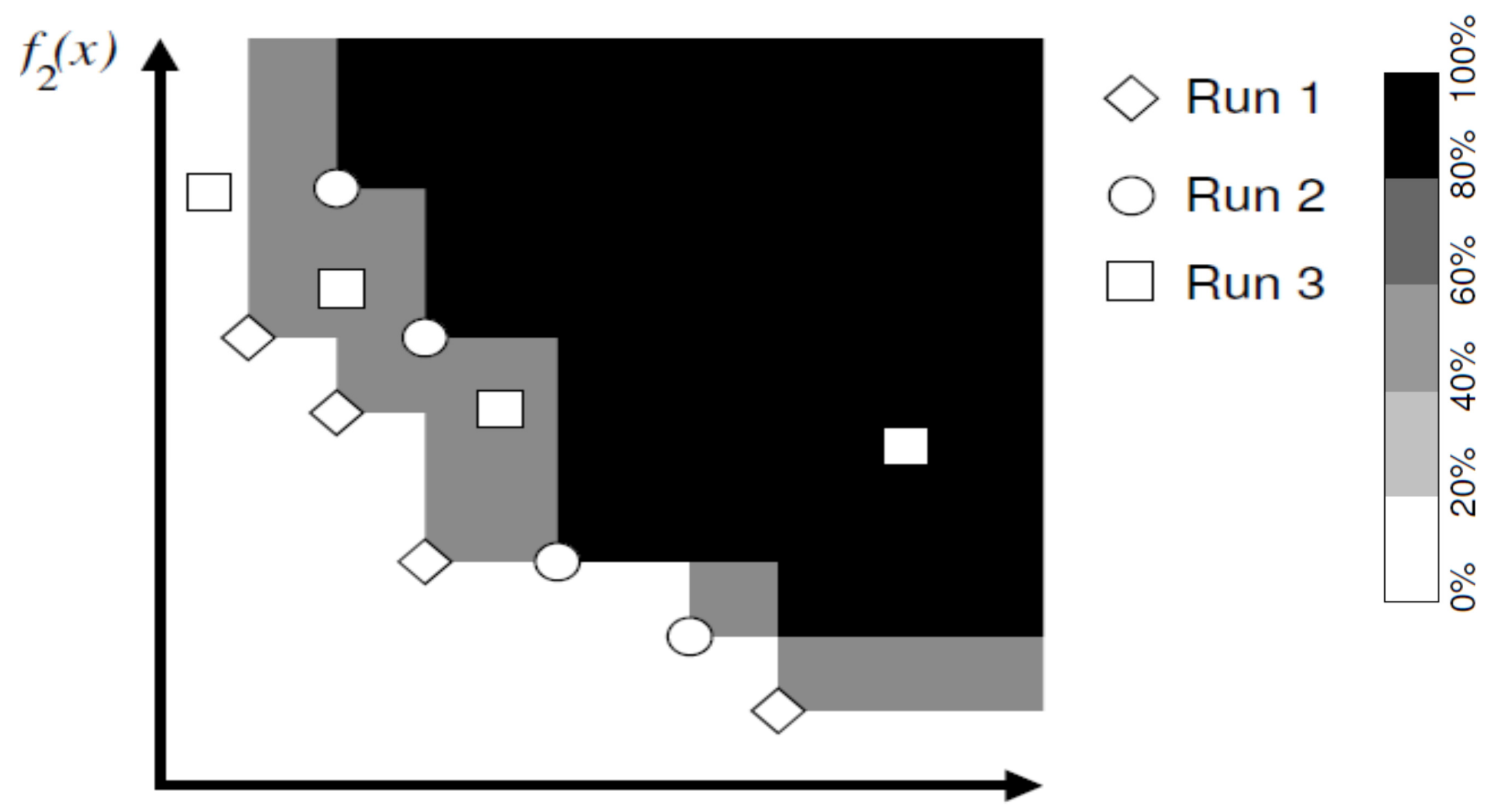

(C) Manuel López-lbáñez [López-lbáñez et al. 2010]

$f_{1}(x)$ 


\section{Empirical Attainment Functions}

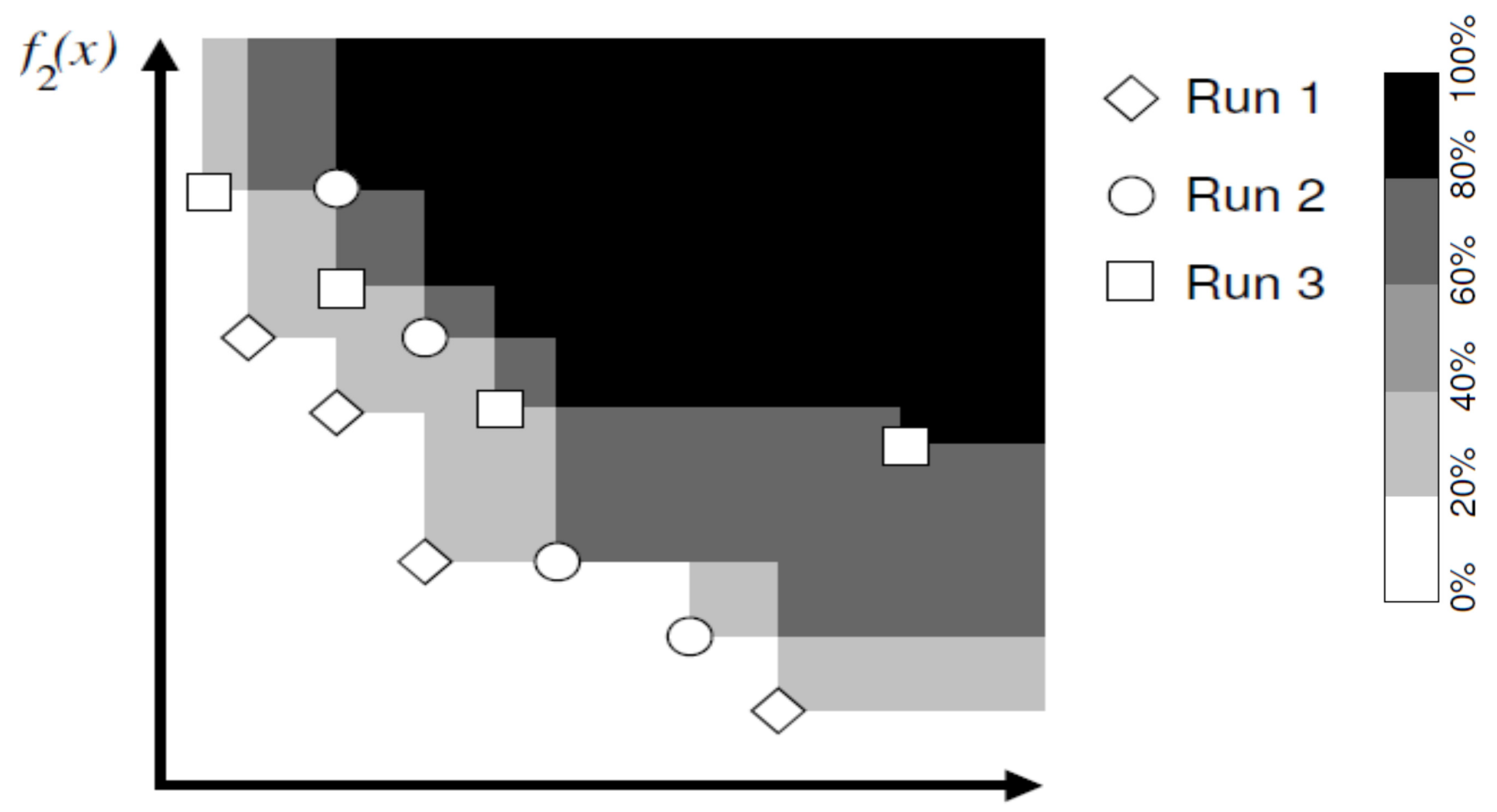

(C) Manuel López-lbáñez

[López-lbáñez et al. 2010]

$f_{1}(x)$ 


\section{Empirical Attainment Functions: Definition}

The Empirical Attainment Function $\alpha(z)$ "counts" how many solution sets $X_{i}$ attain or dominate a vector $z$ at time $T$ :

$$
\alpha_{T}(z)=\frac{1}{N} \sum_{i=1}^{N} \mathbf{1}_{\left\{\chi_{i} \unlhd_{T} Z\right\}}
$$

with $\unlhd_{T}$ being the weak dominance relation between a solution set and an objective vector at time $T$.

Note that $\alpha_{T}(z)$ is the empirical cumulative distribution function of the achieved objective function distribution at time $T$ in the single-objective case ("fixed budget scenario"). 


\section{Empirical Attainment Functions in Practice}

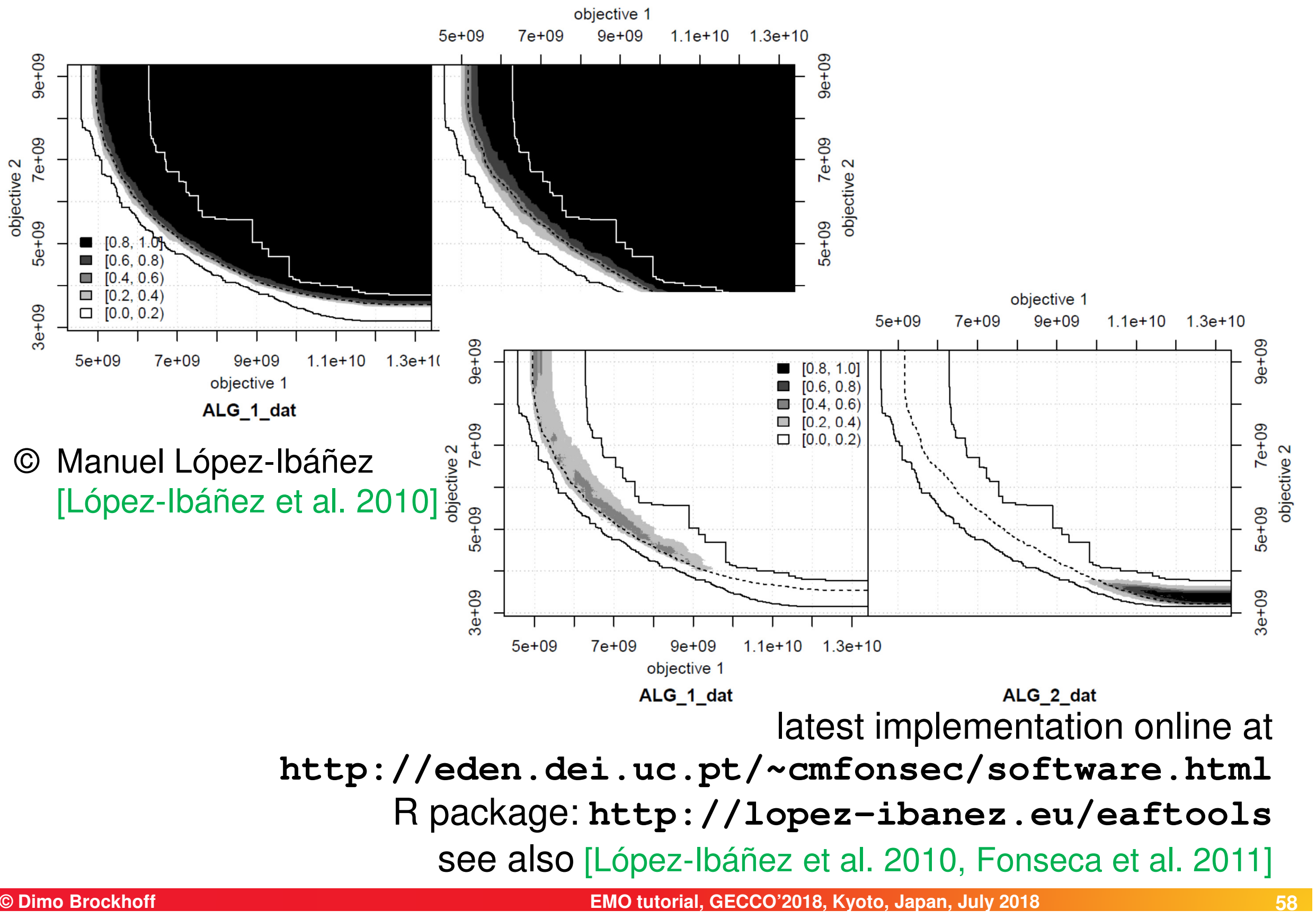




\section{Plotting Average Runtimes}

Note: success probability can be naturally replaced by the average runtime of an artificially restarted algorithm (aRT):

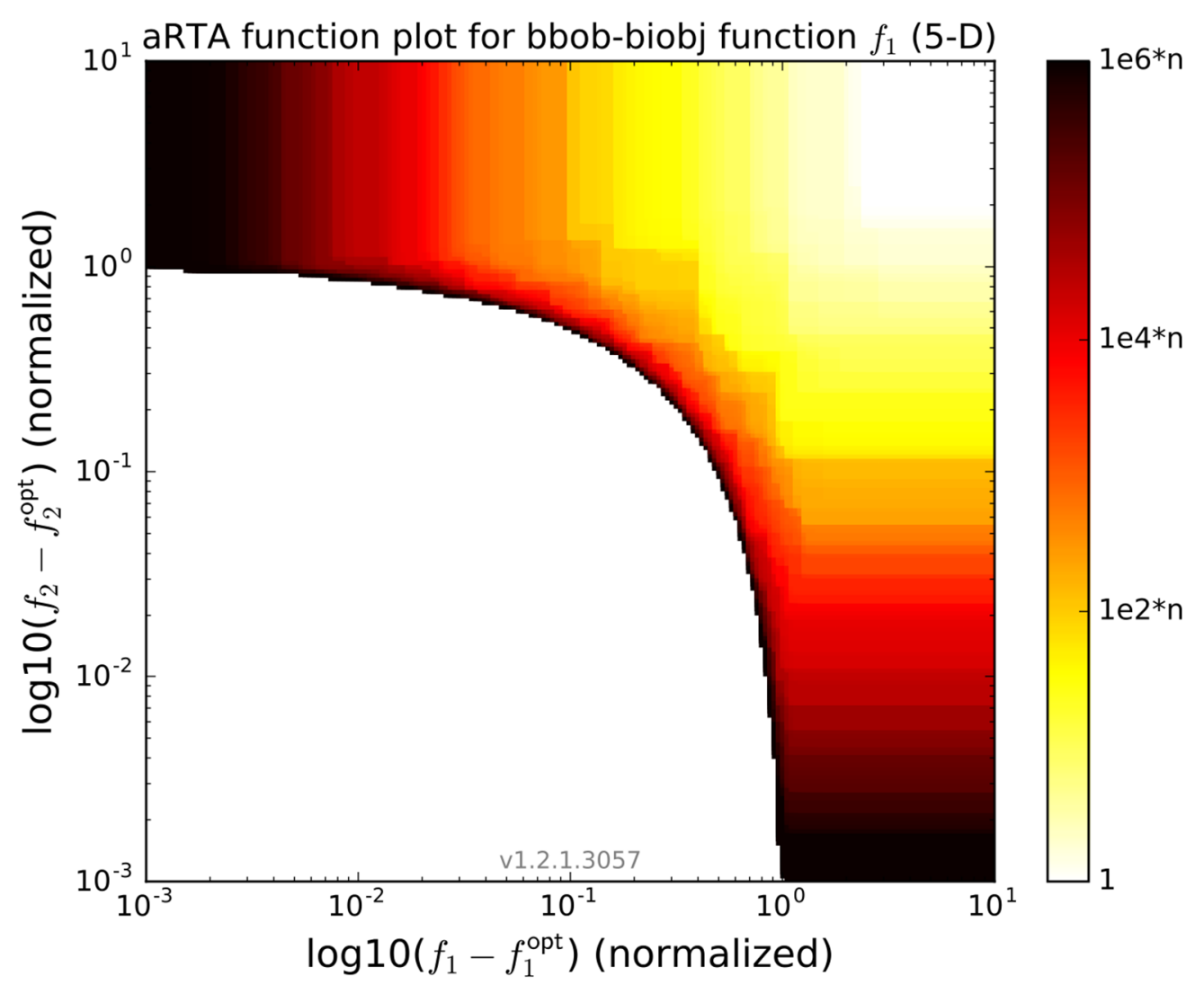

code available at http://github.com/numbbo/coco/ see also [Brockhoff et al. 2017] 


\section{Quality Indicator Approach}

\section{Idea:}

- transfer multiobjective problem into a set problem

- define an objective function ("quality indicator") on sets

- use the resulting total (pre-)order (on the quality values)

\section{Question:}

Can any total (pre-)order be used or are there any requirements concerning the resulting preference relation?

$\Rightarrow$ Underlying dominance relation should be reflected!

$$
A \preceq B: \Leftrightarrow \forall_{y \in B} \exists_{x \in A} x \leq_{\text {par }} y
$$




\section{Refinements and Weak Refinements}

( $\stackrel{\text { ref }}{\preccurlyeq}$ refines a preference relation $\preccurlyeq$ iff

$$
\begin{aligned}
& A \preccurlyeq B \wedge B \npreceq A \Rightarrow A \stackrel{\text { ref }}{\preccurlyeq} B \wedge B \stackrel{\text { ref }}{\nprec} A \quad \text { (better } \Rightarrow \text { better) } \\
& \Rightarrow \text { fulfills requirement }
\end{aligned}
$$

(2) $\stackrel{\text { ref }}{\preccurlyeq}$ weakly refines a preference relation $\preccurlyeq$ iff

$$
\begin{aligned}
& A \preccurlyeq B \wedge B \npreceq A \Rightarrow A \stackrel{\text { ref }}{\preccurlyeq} B \quad \text { (better } \Rightarrow \text { weakly better) } \\
& \Rightarrow \text { does not fulfill requirement, but } \stackrel{\text { ref }}{\preccurlyeq} \text { does not contradict } \preccurlyeq
\end{aligned}
$$

! sought are total refinements... 


\section{Example: Refinements Using Indicators}

$$
A \stackrel{\text { ref }}{\preccurlyeq} B: \Leftrightarrow I(A) \geq I(B)
$$

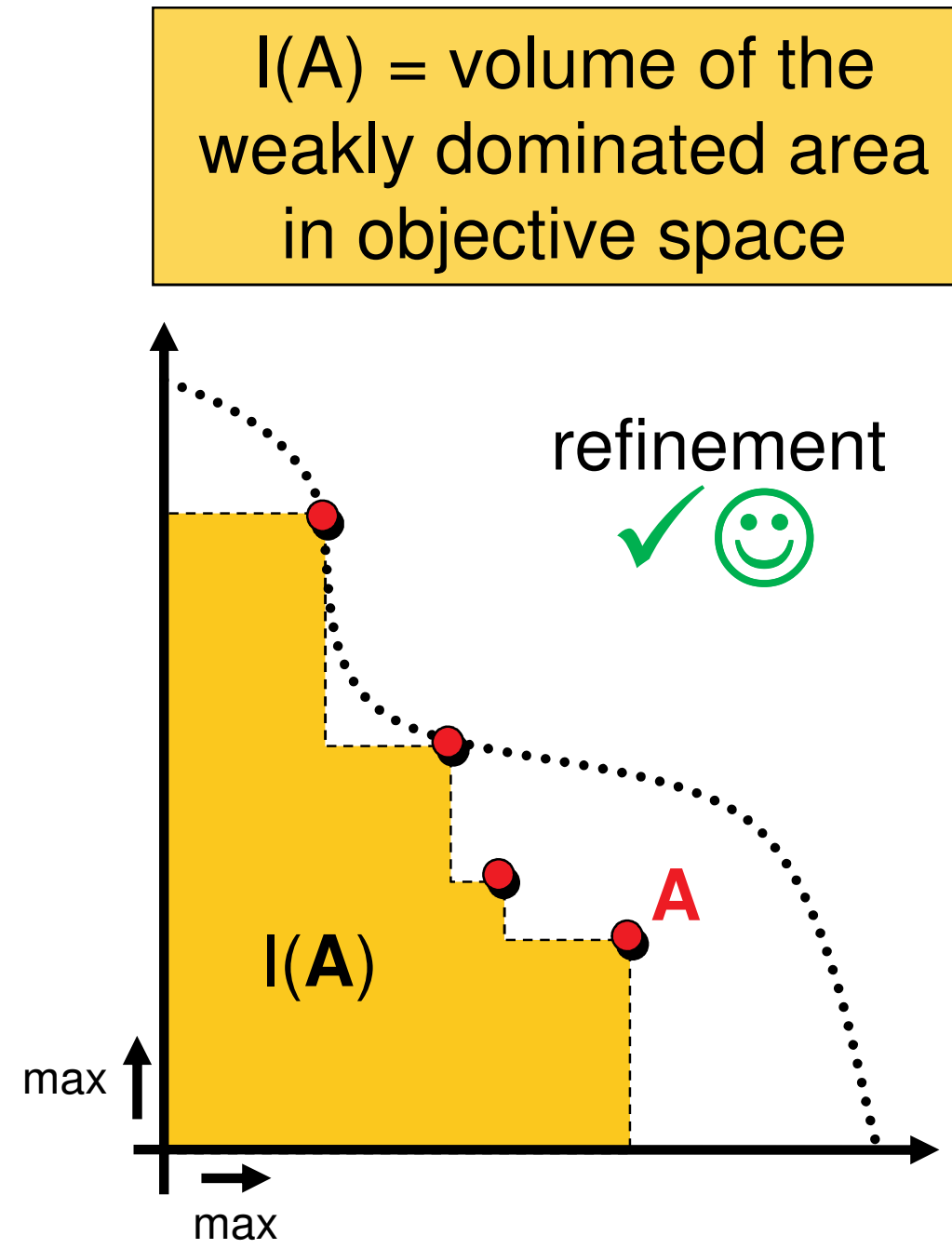

unary hypervolume indicator

$$
A \stackrel{\text { ref }}{\preccurlyeq} B: \Leftrightarrow I(A, B) \leq I(B, A)
$$

$\mathrm{I}(\mathrm{A}, \mathrm{B})=$ how much needs $A$ to be moved to weakly dominate $B$

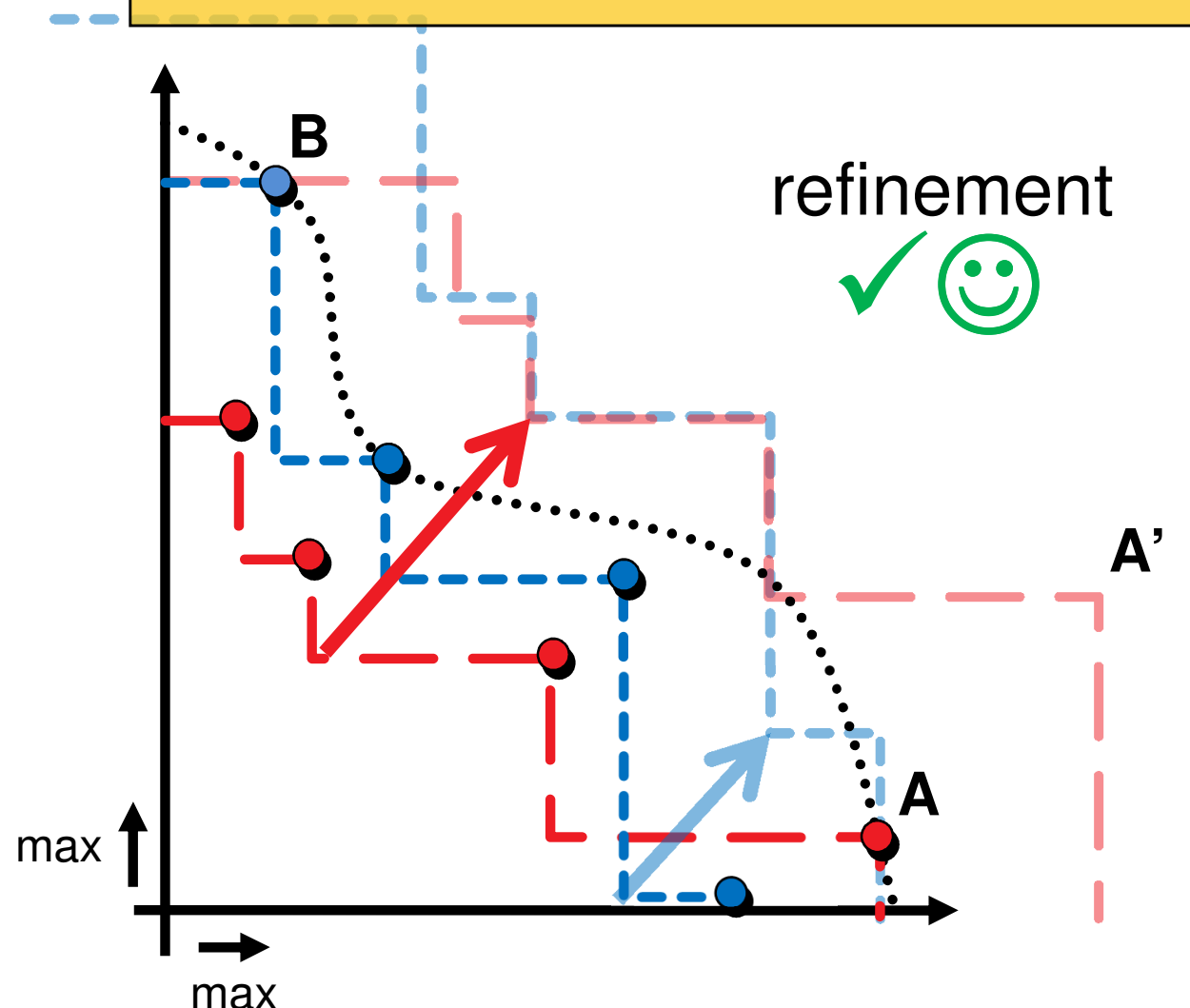

binary epsilon indicator 


\section{Example: Weak Refinement / No Refinement}

$$
A \stackrel{\text { ref }}{\preccurlyeq} B: \Leftrightarrow I(A, R) \leq I(B, R)
$$

$\mathrm{I}(\mathrm{A}, \mathrm{R})=$ how much needs $\mathrm{A}$ to be moved to weakly dominate $R$

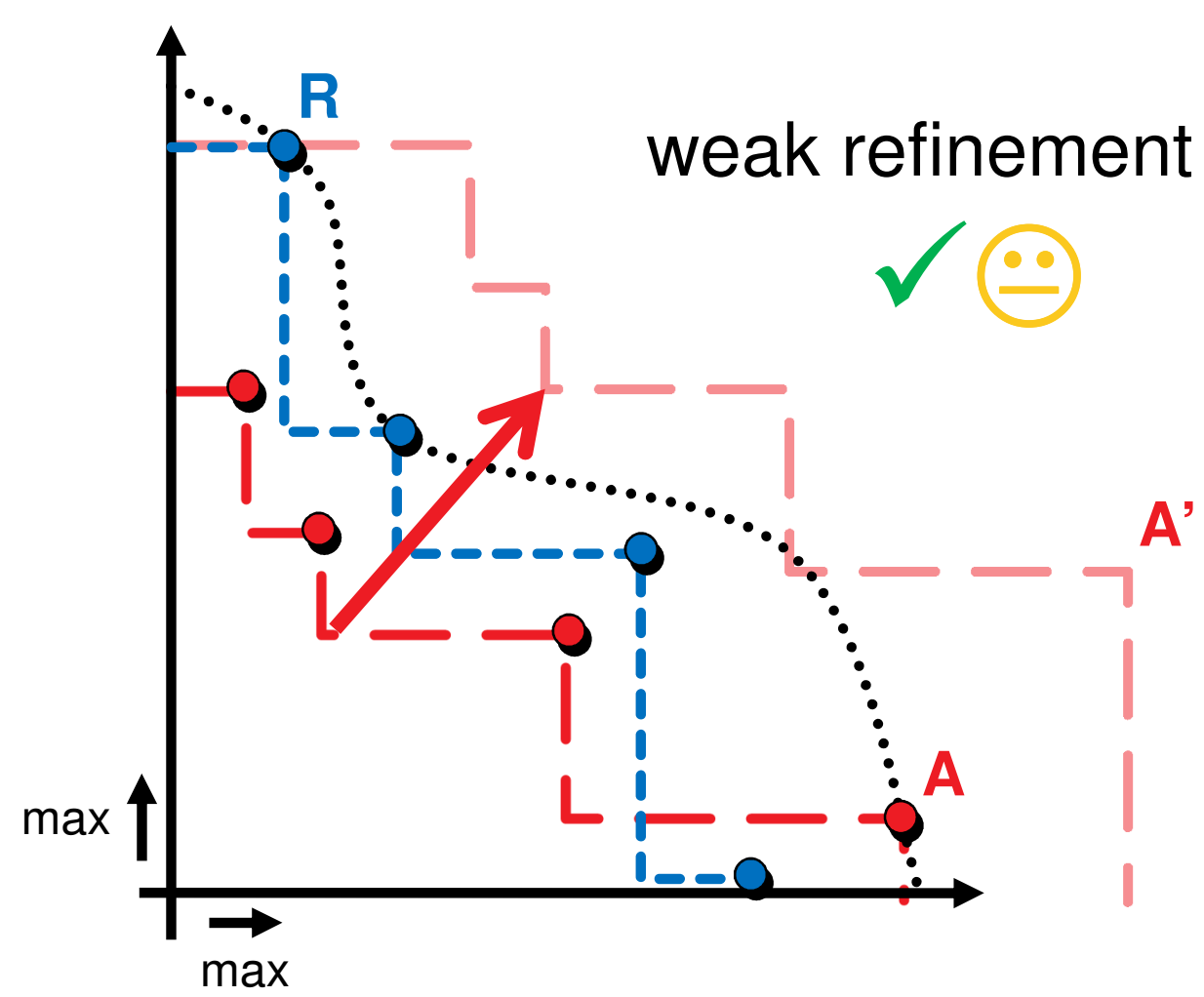

unary epsilon indicator

$$
A \stackrel{\text { ref }}{\preccurlyeq} B: \Leftrightarrow I(A) \leq I(B)
$$

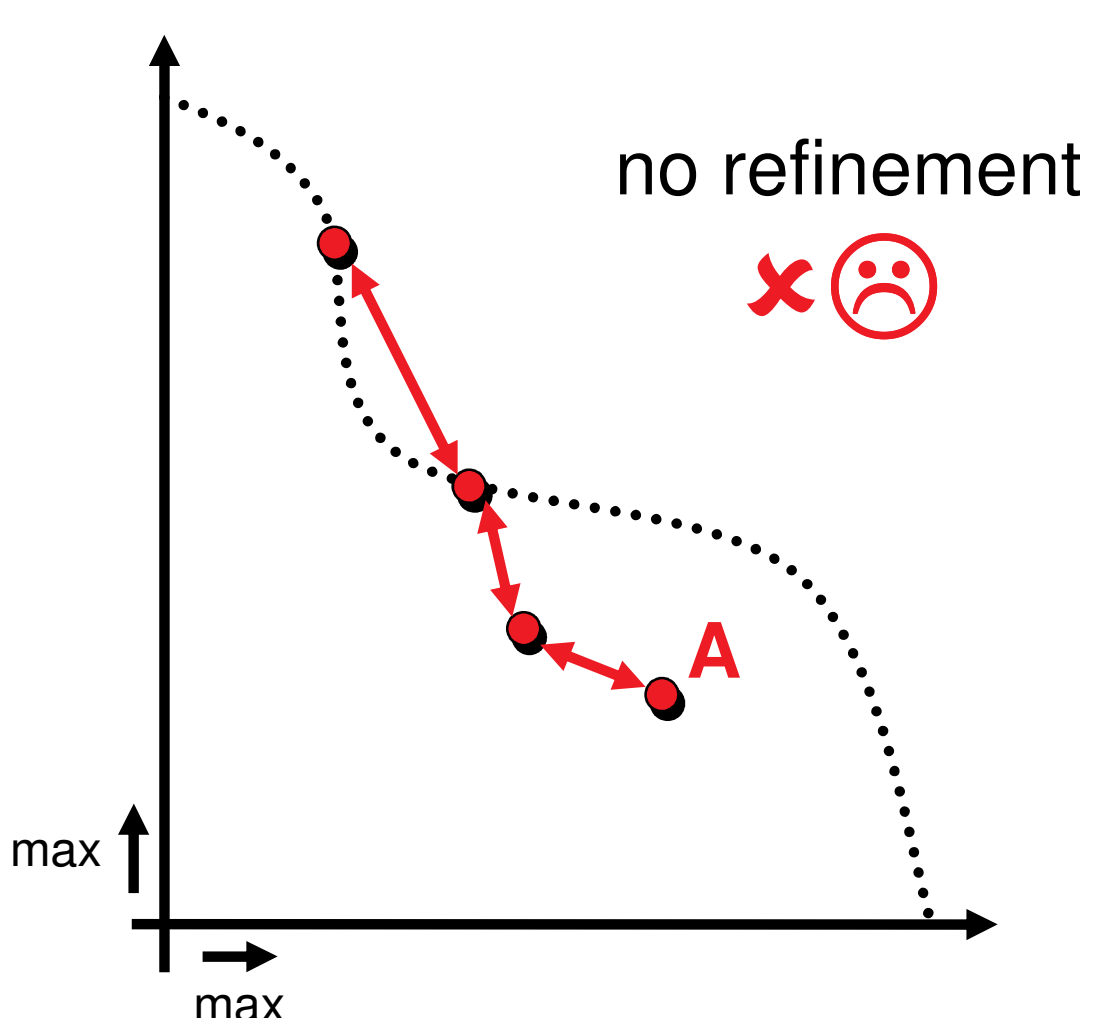

unary diversity indicator 


\section{Quality Indicator Approach}

Goal: compare two Pareto set approximations A and B

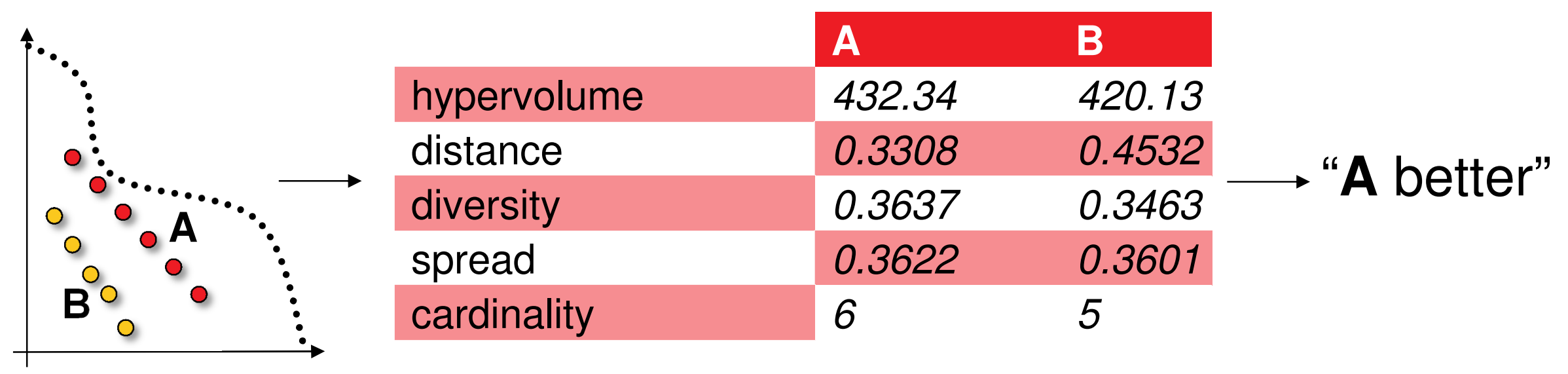

Comparison method $\mathrm{C}=$ quality measure(s) + Boolean function
quality
$A, B \stackrel{\text { measure }}{\longrightarrow} \quad \| R^{n}$
Boolean
reduction
function
statement

\section{interpretation}




\section{Example: Box Plots}

\section{epsilon indicator hypervolume $\quad R$ indicator}

IBEA NSGA-IISPEA2 IBEA NSGA-IISPEA2 IBEA NSGA-IISPEA2
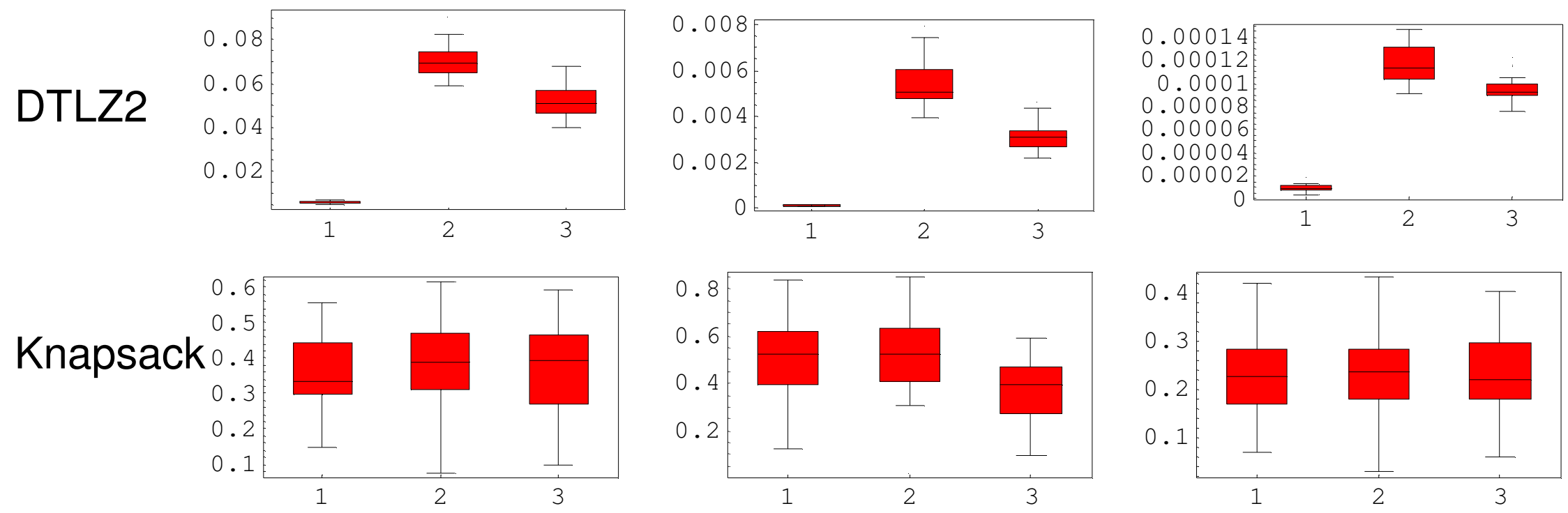

ZDT6
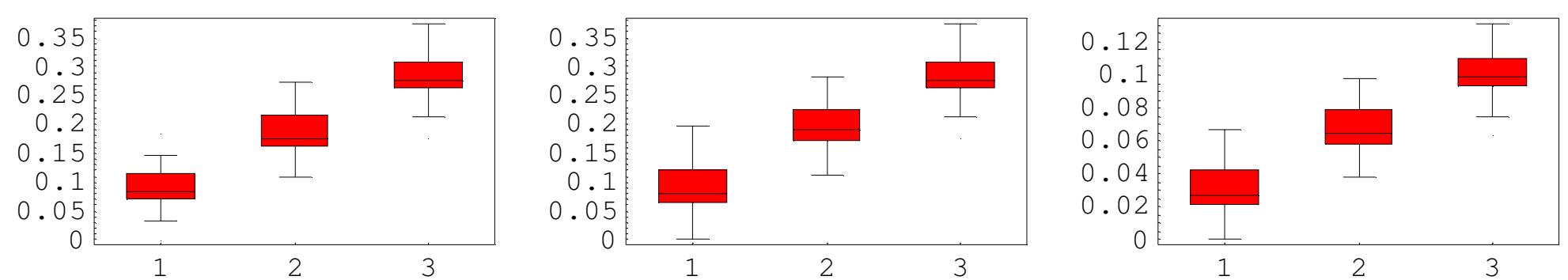


\section{Statistical Assessment (Kruskal Test)}

ZDT6

Epsilon

is better

\begin{tabular}{|c|c|c|c|}
\hline 1 & IBEA & NSGA2 & SPEA2 \\
\hline IBEA & & $\sim 0 \quad \bigcirc$ & $\sim 0$ \\
\hline NSGA2 & 1 & & $\sim 0$ \\
\hline SPEA2 & 1 & 1 & \\
\hline
\end{tabular}

Overall $p$-value $=6.22079 \mathrm{e}-17$

Null hypothesis rejected (alpha 0.05 )

\section{DTLZ2}

$\mathrm{R}$

is better

than

\begin{tabular}{l|l|ll|ll}
$\longrightarrow$ & IBEA & \multicolumn{2}{|l|}{ NSGA2 } & \multicolumn{2}{|l}{ SPEA2 } \\
\hline IBEA & & $\sim 0$ & $\bigodot$ & $\sim 0$ & $\odot$ \\
\hline NSGA2 & 1 & & & 1 & \\
\hline SPEA2 & 1 & $\sim 0$ & $\ominus$ & &
\end{tabular}

Overall p-value $=7.86834 \mathrm{e}-17$

Null hypothesis rejected (alpha 0.05)

Knapsack/Hypervolume: $\mathrm{H}_{0}=$ No significance of any differences 


\section{Automated Benchmarking}

- State-of-the-art in single-objective optimization: Blackbox Optimization Benchmarking (BBOB) with COCO platform

https://github.com/numbbo/coco

- Release of a bi-objective test suite at BBOB-2016 workshop

- Focus on target-based runlengths

- gives (nearly) anytime, interpretable results

- defines problem=(test function instance, single-objective goal e.g. min. indicator difference to reference set, target precision)

- reports average runtimes (aRT) to reach target precision

- COCO provides data profiles, scaling plots, scatter plots, tables, statistical tests, etc. automatically 


\section{Exemplary BBOB-2016 Results}

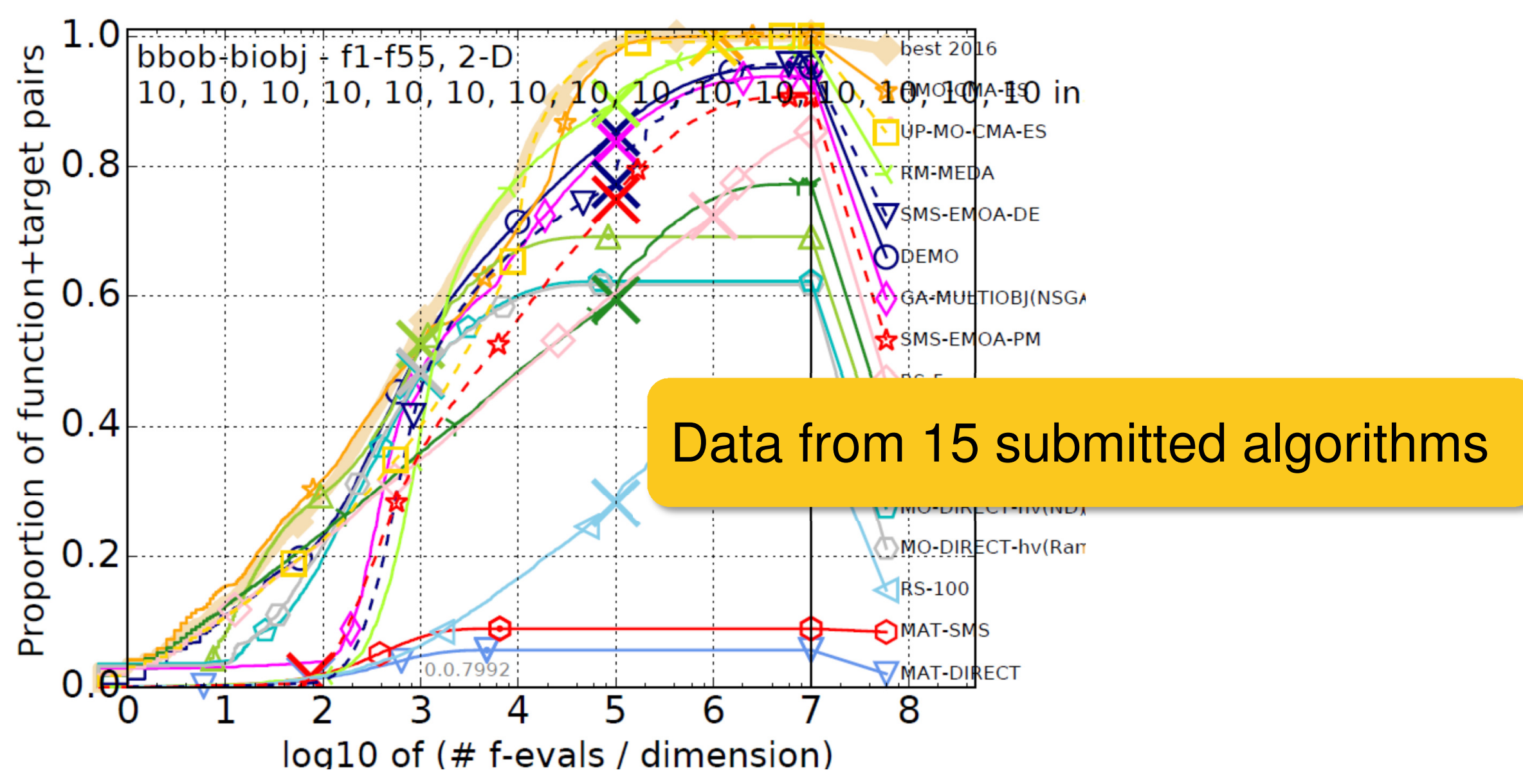




\section{Exemplary BBOB-2016 Results}

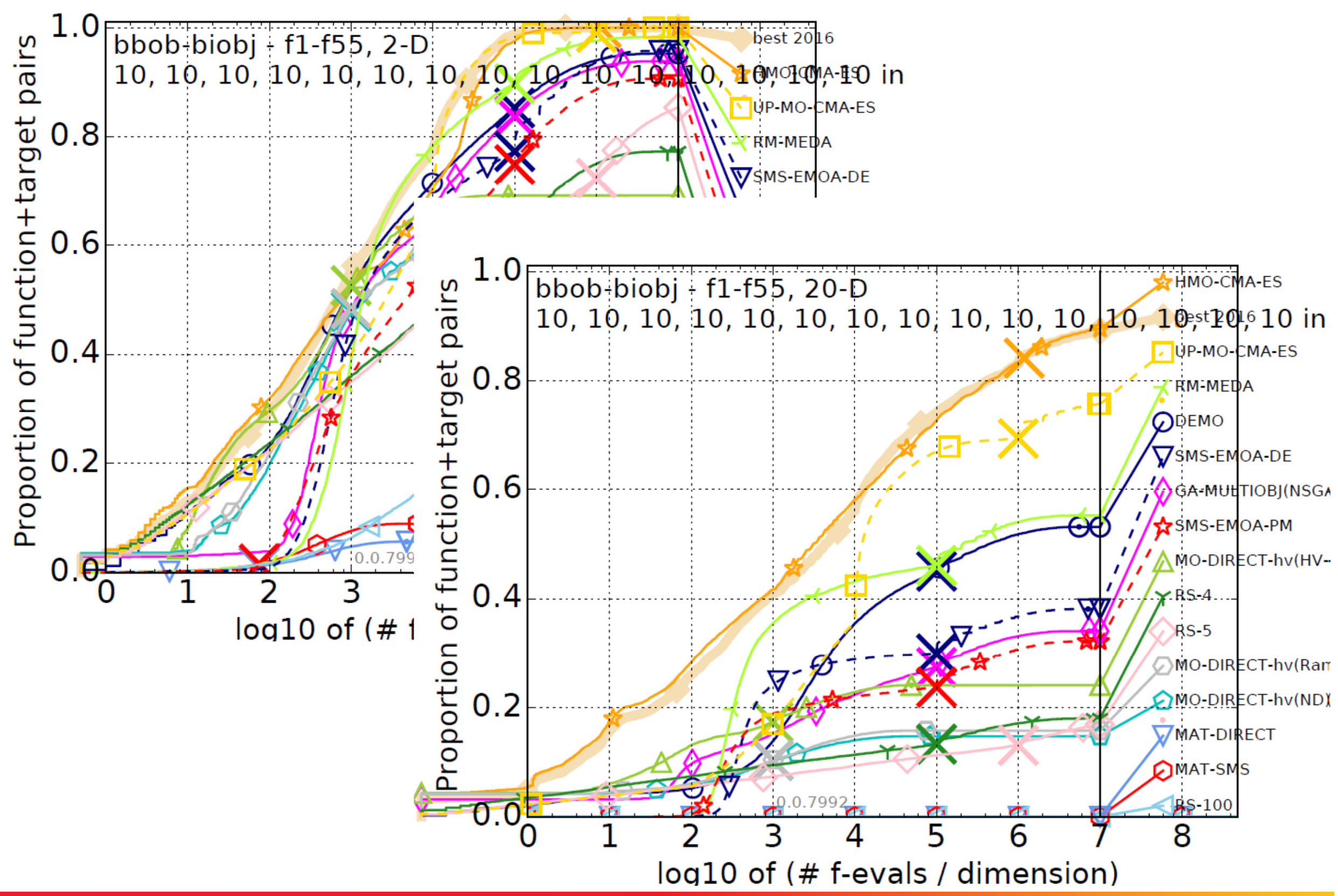




\section{A Few Recommendations}

- always display everything you have

- look at single runs

- do each experiment at least twice

(= look at the variance of your results)

- as quality indicators, use hypervolume, R2, or epsilon indicator

- see also the tutorial by Nikolaus Hansen on this topic (not restricted to single-objective optimization!) 


\section{Overview}

The Big Picture

Basic Algorithm Design Principles and Concepts

Performance Assessment and Benchmarking

Preference Articulation 


\section{Articulating User Preferences During Search}

What we thought: EMO is preference-less

Search before decision making: Optimization is performed without any preference information given. The result of the search process is a set of

(ideally Pareto-optimal) candidate solutions from which the final choice is made by the DM.

What we learnt: EMO just uses weaker preference information

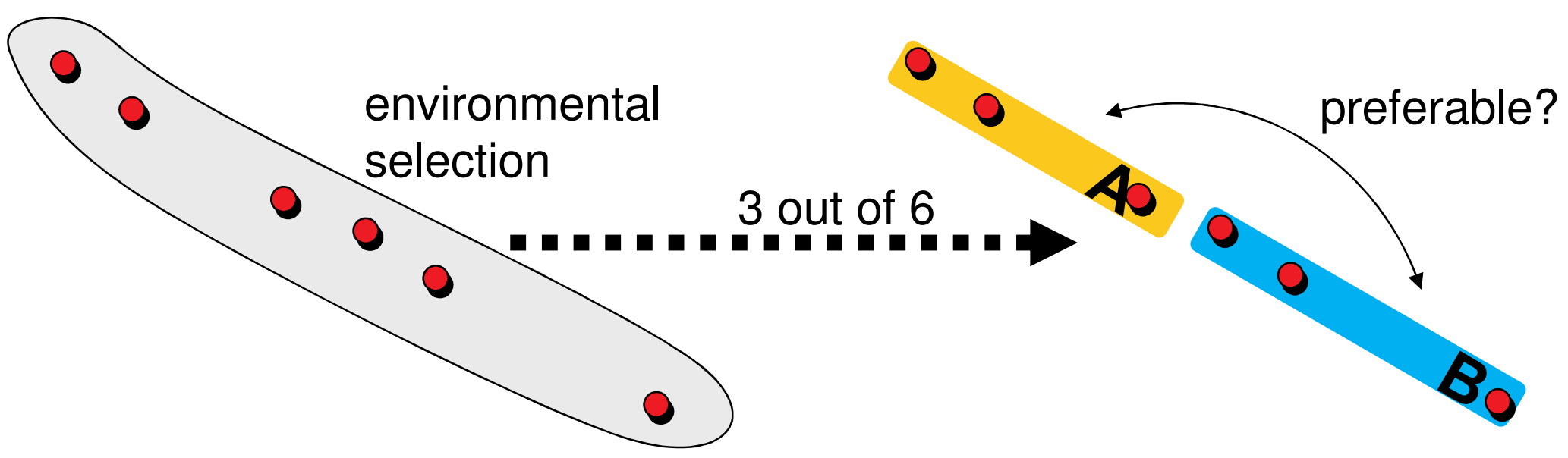




\section{Incorporation of Preferences During Search}

Nevertheless...

- the more (known) preferences incorporated the better

- in particular if search space is large

[Branke and Deb 2004] [Branke 2008] [Bechikh et al. 2015]

( Refine/modify dominance relation, e.g.:

- using goals, priorities, constraints [Fonseca and Fleming 1998a,b]

- using different types of dominance cones [Branke and Deb 2004]

2 Use quality indicators, e.g.:

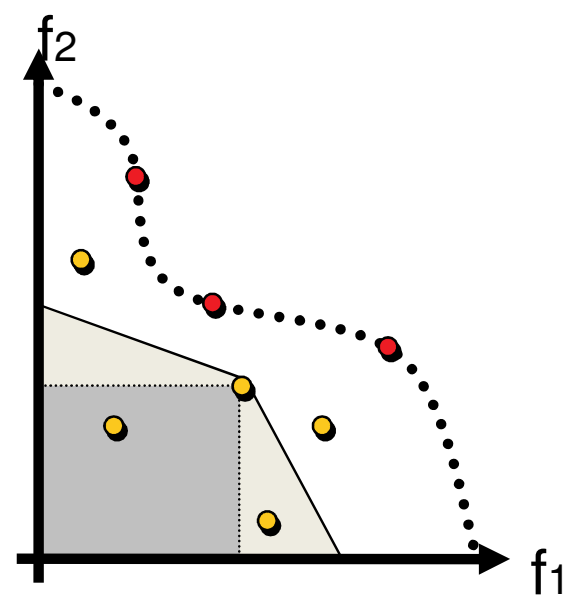

- based on reference points and directions [Deb and Sundar 2006, Deb and Kumar 2007]

- based on the hypervolume indicator [Brockhoff et al. 2013] [Wagner and Trautmann 2010]

- based on the R2 indicator [Trautmann et al. 2013] 


\section{Example: Weighted Hypervolume Indicator}

$$
I_{H}^{W}(A)=\int_{\vec{i}}^{\vec{r}} \operatorname{wa}(\vec{z}) d \vec{z}
$$

weighted

hypervolume

[Brockhoff et al. 2013]

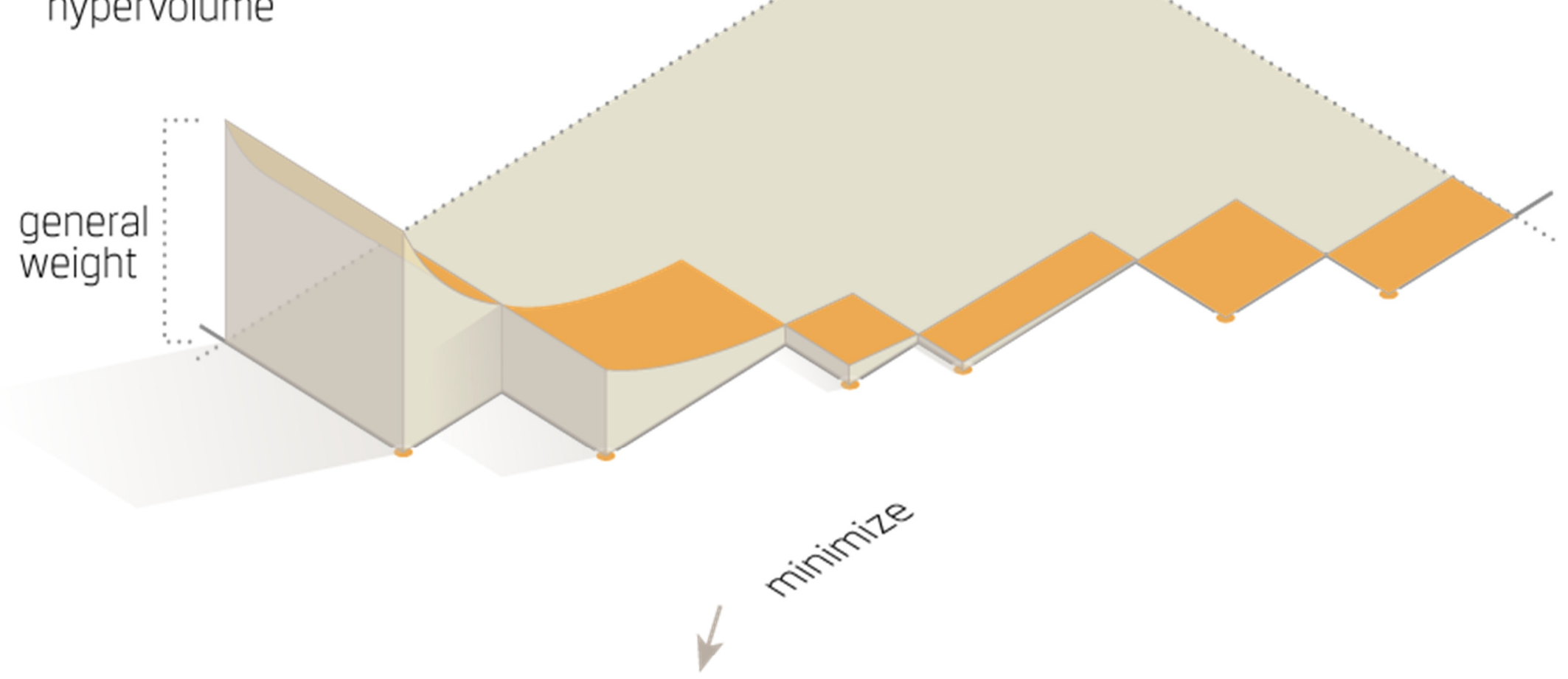




\section{Weighted Hypervolume in Practice}

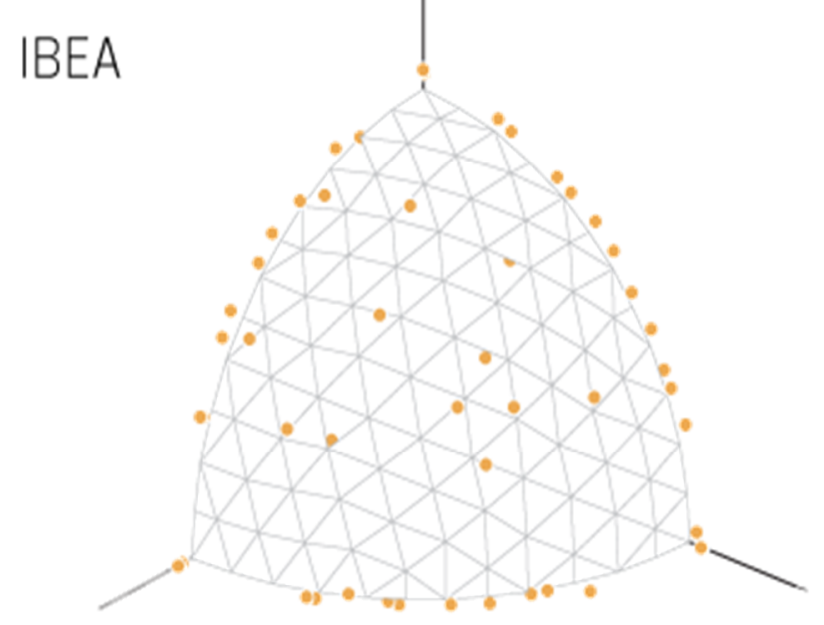

IBEA
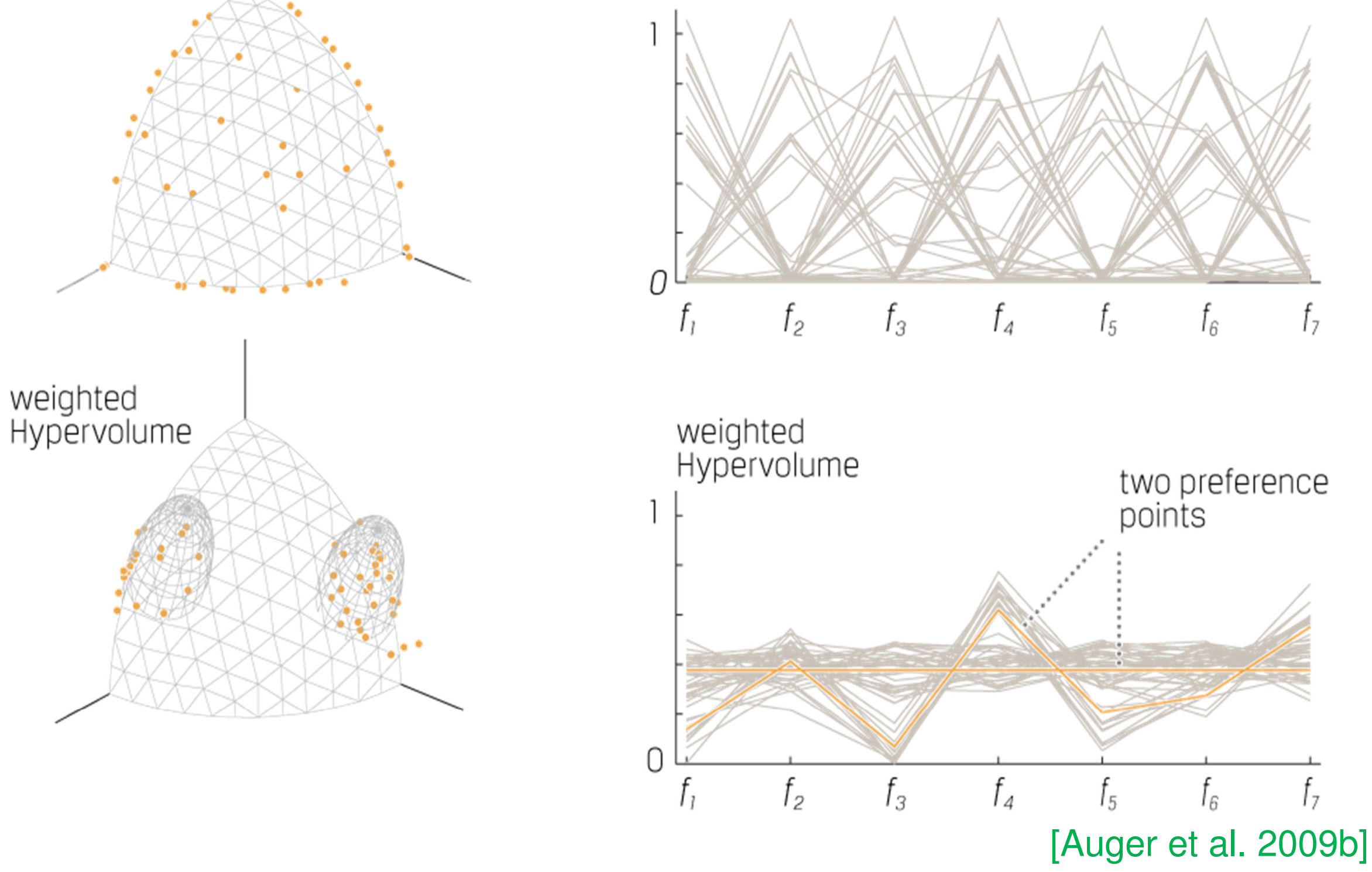


\section{Example: Desirability Function (DF)-SMS-EMOA}

Shape of the untransformed Pareto front

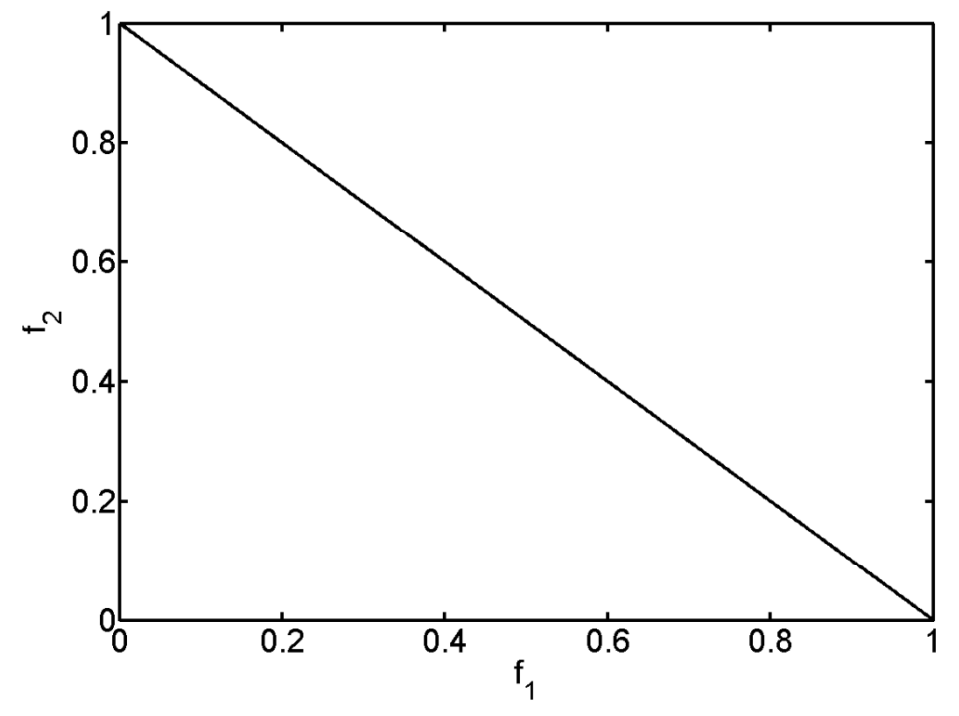

Shape of the transformed front for

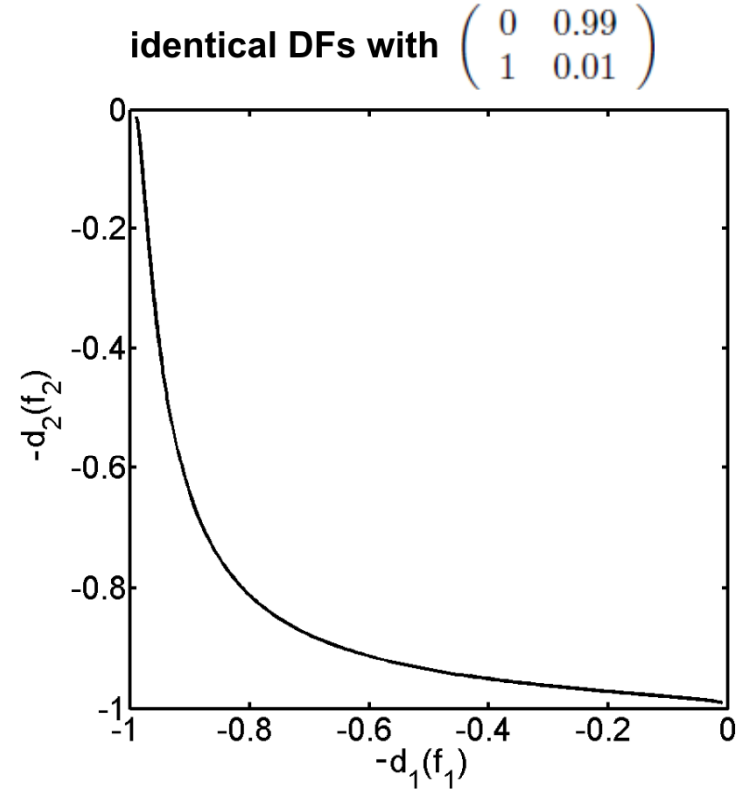

Shape of the transformed front for

identical DFs with $\left(\begin{array}{cc}0 & 0.99 \\ 0.75 & 0.01\end{array}\right)$

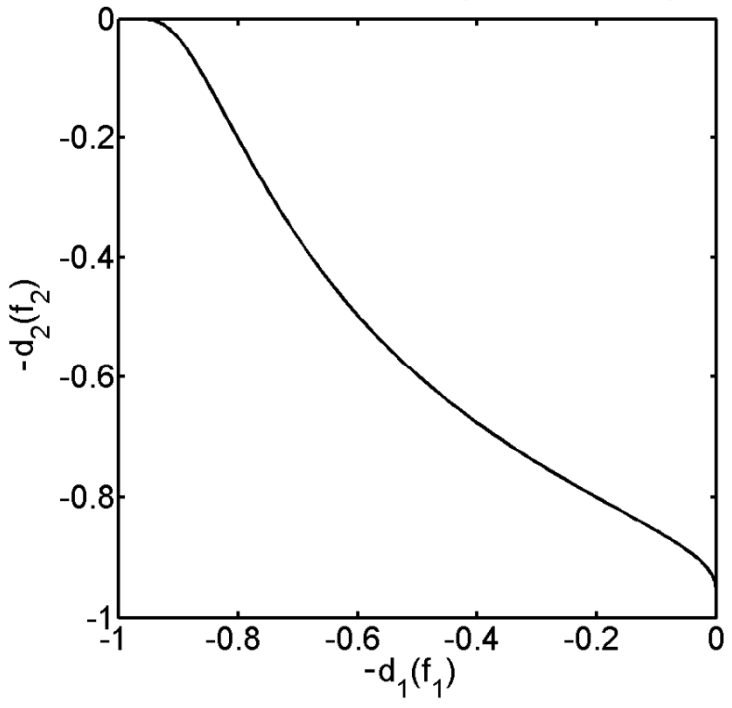

[Wagner and Trautmann 2010]

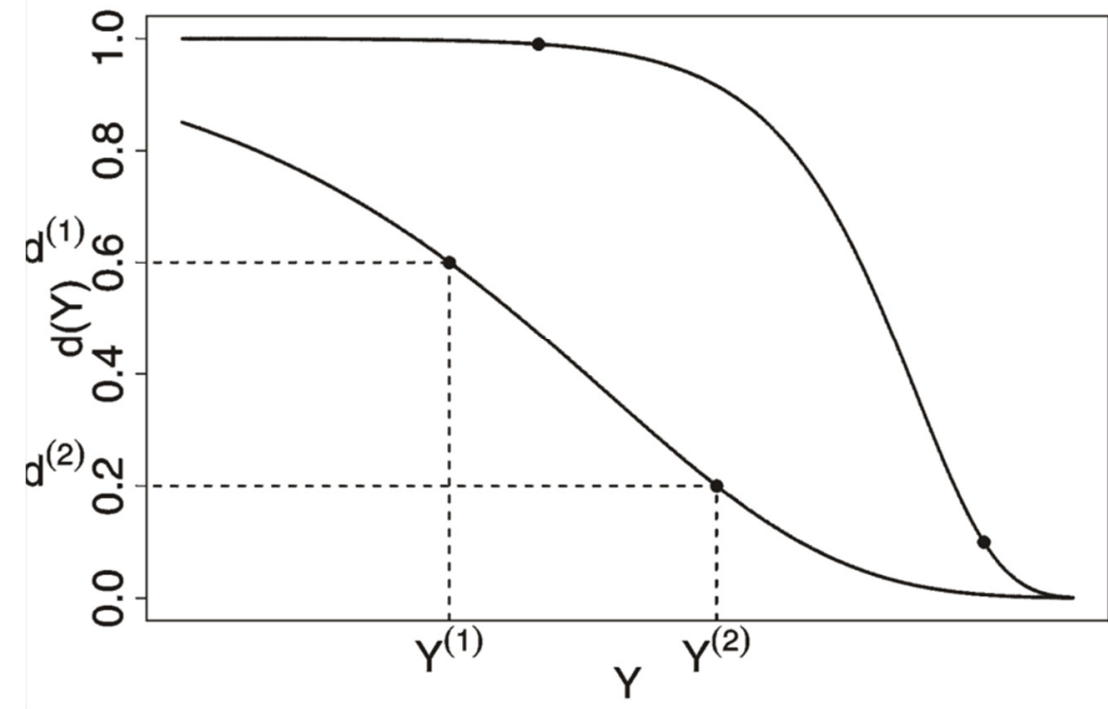

Shape of the transformed front for

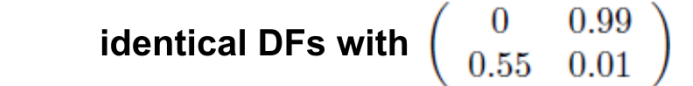




\section{DF-SMS-EMOA in Practice}
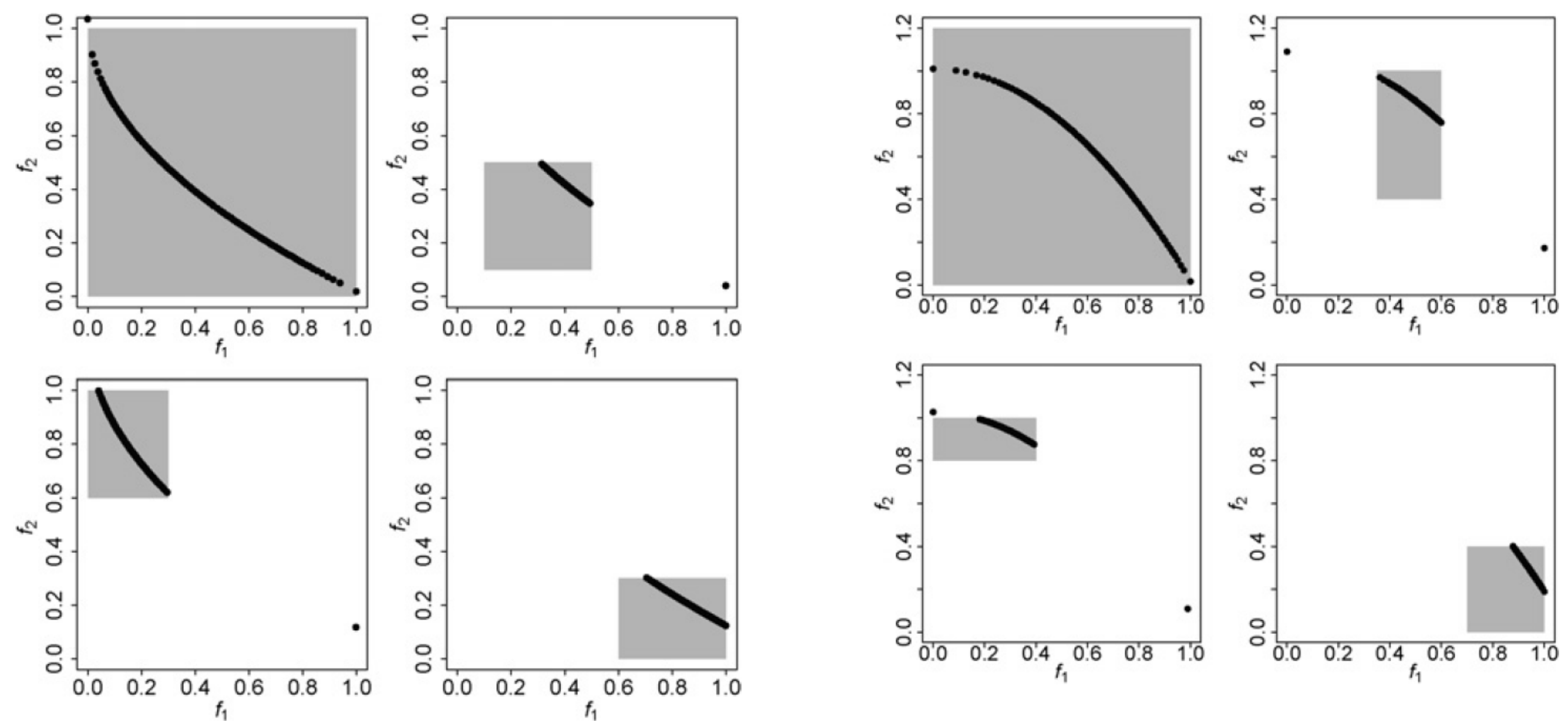


\section{Example: R2-EMOA}

\section{Concept}

Integration of preferences by varying the scalarizing functions

\section{Position of ideal point}
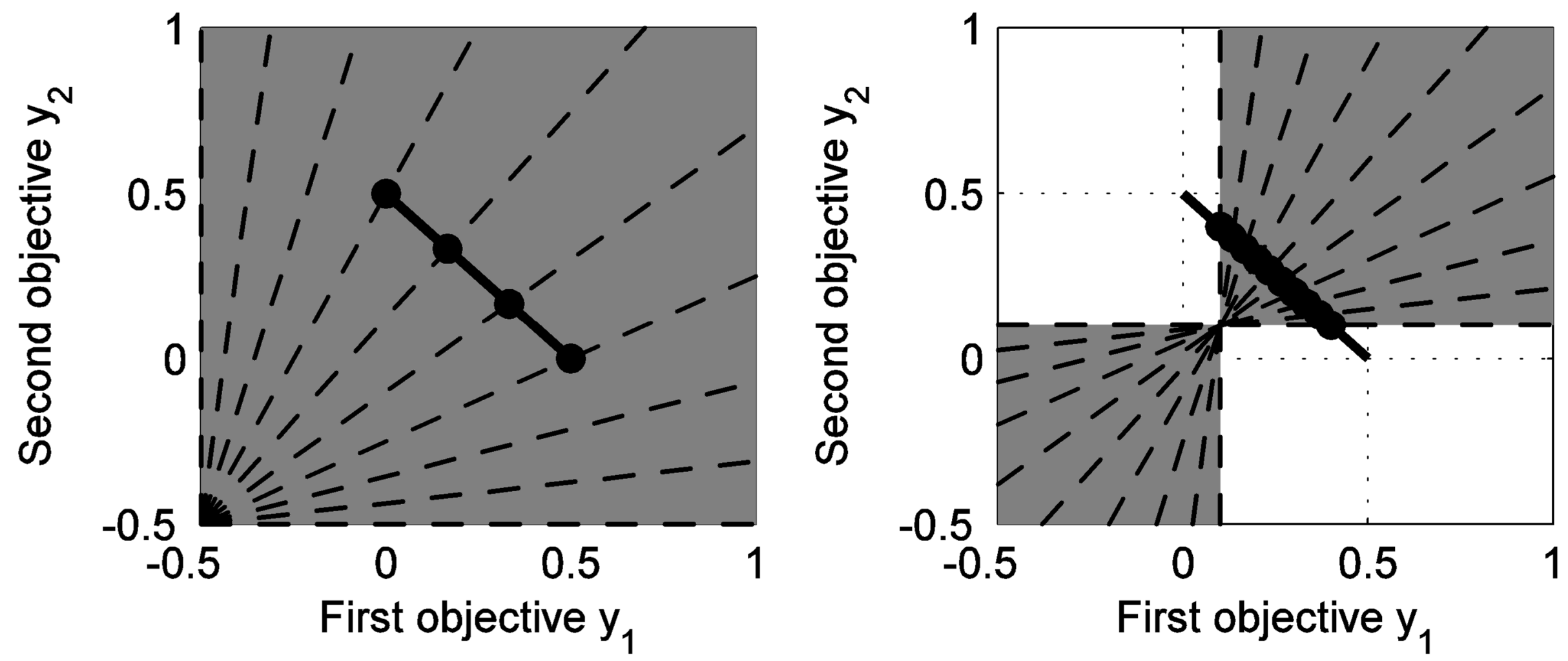


\section{Example: R2-EMOA}

\section{Concept}

Integration of preferences by varying the scalarizing functions

Restriction of the weight space

\section{ZDT1}

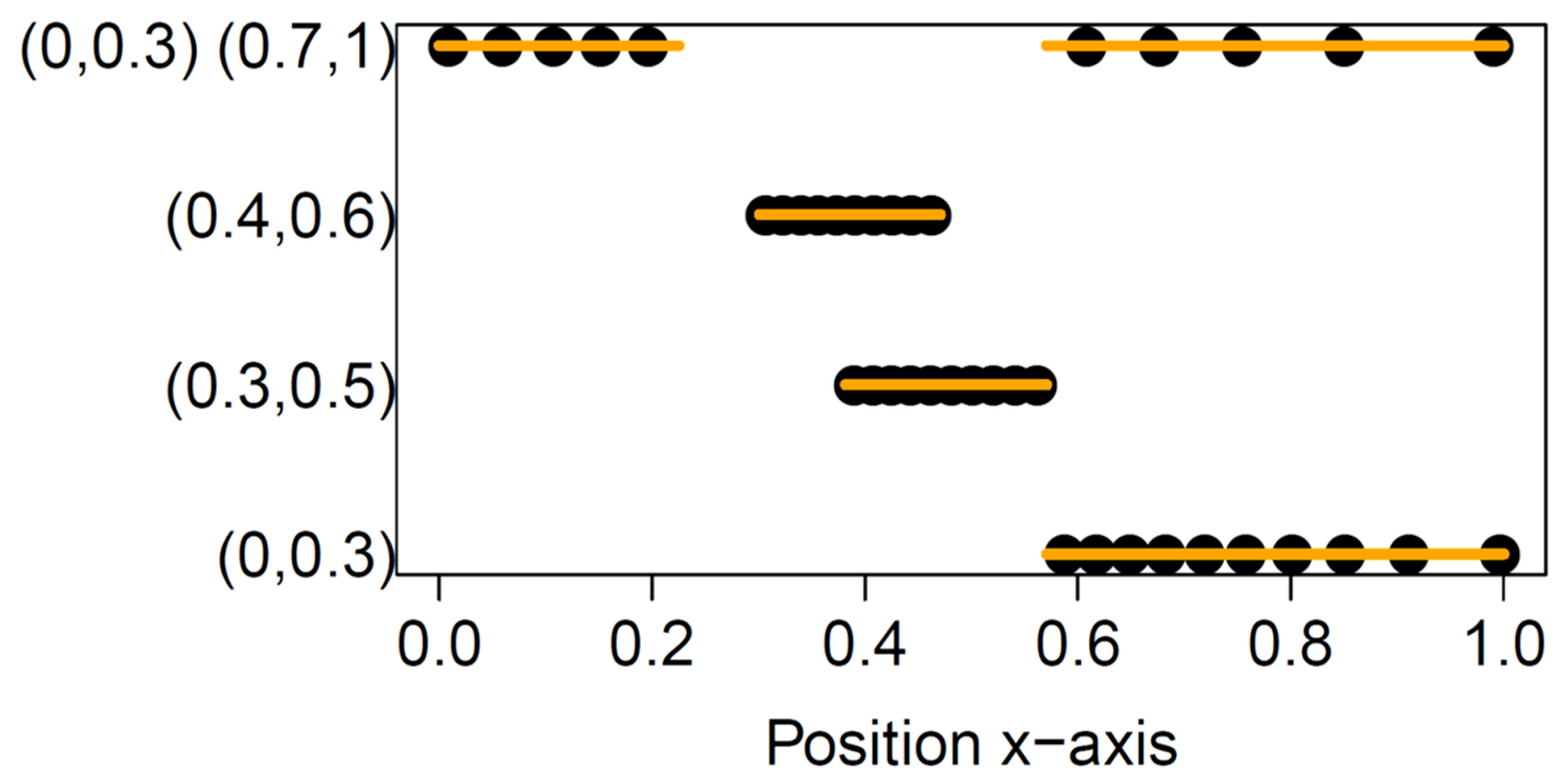




\section{Interactive Approaches}

Successive Preference Articulation = Interactive EMO

- recent interest of both EMO and MCDM community

- important in practice

Examples

- first interactive EMO: [Tanino et al. 1993]

- good overview: [Jaszkiewicz and Branke 2008]

- more recent work: [Brockhoff et al. 2014] [Branke et al. 2014]

\section{Issues/Open Questions}

- realistic scenarios/value functions

- evaluation of interactive algorithms [López-lbáñez and Knowles 2015] 


\section{Conclusions: EMO as Interactive Decision Support}

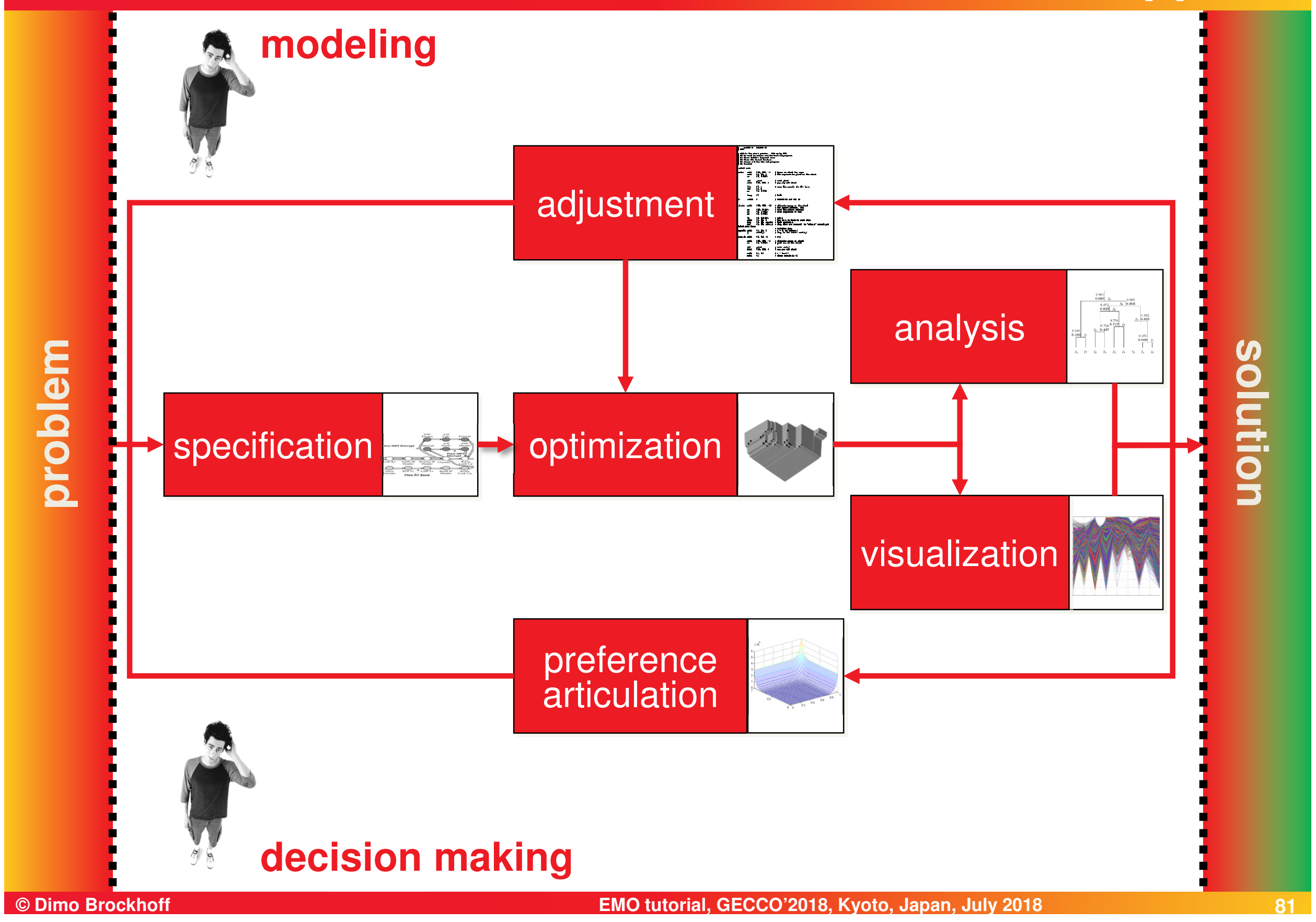




\section{The EMO Community}

\section{Links:}

- EMO mailing list: https://lists.dei.uc.pt/mailman/listinfo/emo-list

- MCDM mailing list: http://lists.jyu.fi/mailman/listinfo/mcdm-discussion

- EMO bibliography: http://www.lania.mx/ ccoel/o/EMOO/

- EMO conference series: http://www.dep.uminho.pt/EMO2015/

\section{Books:}

- Multi-Objective Optimization using Evolutionary Algorithms Kalyanmoy Deb, Wiley, 2001

- Evolutionary Algorithms for Solving Multi Evolutionary Algorithms for Solving Multi-Objective Problems Objective Problems, Carlos A. Coello Coello, David A. Van Veldhuizen \& Gary B. Lamont, Kluwer, $2^{\text {nd }}$ Ed. 2007

- Multiobjective Optimization-Interactive and Evolutionary Approaches, J. Branke, K. Deb, K. Miettinen, and R. Slowinski, editors, volume 5252 of LNCS. Springer, 2008 [(still) many open questions!]

- $\quad$ and more... 


\section{Software}

\section{ETH

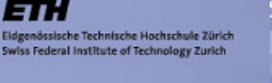

\section{A}

5. Principles and
Documentation

Q PISA for Beginners

Downloads

Performance
Assessment

Write and Submit a

Publications, Bugs,
Contact \& License

$I \pi$

Computer Engineering and
Networks Laboratory
Search Qubmin

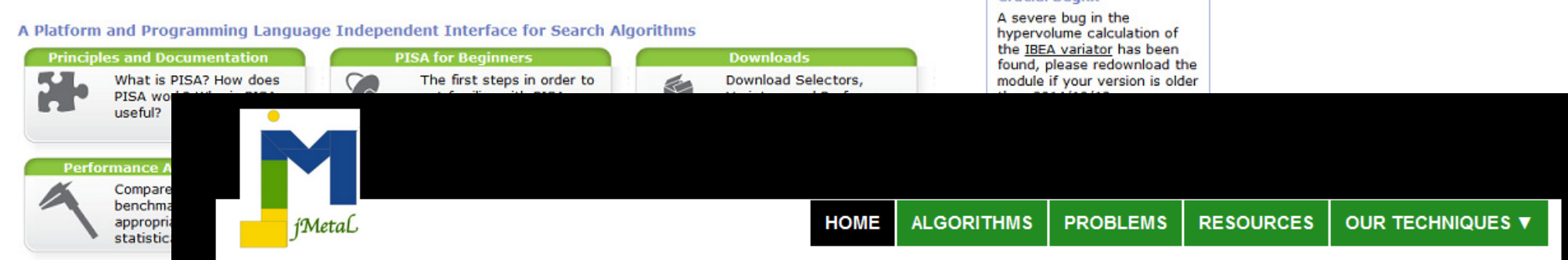

Welcome to the jMetal Web Site

jMetal is ... Summary of features

Download from 5ourcefarge

jMetal stands for Metaheuristic Algorith in Java, and it is an object-orien Java-based framework for multi-objec

You can use it to ...

The object-oriented architecture of framework and the included features allow to: experiment with the provided classic state-of-the-art techniques, develop your algorithms, solve your optimization proble integrate jMetal in other tools, etc.

Our motivation is

The motivation drivinn us is to nrnvide

\section{Home Examples Downloads Documentation Support Donate \\ 9. MOEA Framework \\ A Free and Open Source Java Framework for Multiobjective Optimization}

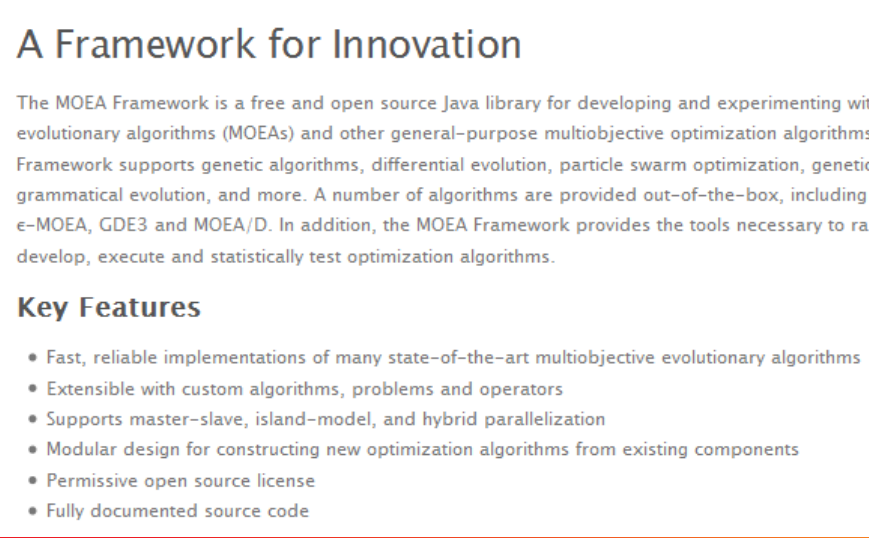

Downloads

Current Version: 2.4

- demo application

:- compiled binaries

:• source Code

:• user manual

Using Maven? Add our dependen Looking for a previous release?

License

- Permissive open source licen

Licensed under the GNU Lesser Ceneral Public License. 


\section{Software}

()

\section{github.com/numbbo/coco/}

numbbo / coco

«Code (1) Issues 115 in Pull requests 1 in Pulse Graphs

Numerical Black-Box Optimization Benchmarking Framework http://coco.gforge.inria.fr/ — Edit

\begin{tabular}{|c|c|c|c|c|c|c|}
\hline \multicolumn{2}{|c|}{ (1) 7,902 commits } & \multicolumn{3}{|l|}{ \& 12 branches } & \multicolumn{2}{|c|}{213 contributors } \\
\hline Branch: master & New pull request & & Create new file & Upload files & Find file & Clone or download - \\
\hline \multicolumn{5}{|c|}{1 brockho committed on GitHub Merge pull request \#1075 from numbbo/development $\quad$... } & \multicolumn{2}{|c|}{ Latest commit $\theta \mathrm{cbb} 7 \mathrm{db}$ on 10 Jun } \\
\hline \multicolumn{2}{|c|}{ code-experiments } & \multicolumn{3}{|c|}{ Merge pull request \#1071 from ttusar/debug } & & a month ago \\
\hline \multicolumn{2}{|c|}{ code-postprocessing } & \multicolumn{3}{|c|}{ further clean up of postprocessing output, } & & a month ago \\
\hline \multicolumn{2}{|c|}{ code-preprocessing/archive-update } & \multicolumn{3}{|l|}{ Added empty last lines. } & & a month ago \\
\hline \multicolumn{2}{|l|}{ docs } & \multicolumn{3}{|c|}{ updated reference to biobjective perf-assessment paper on arXiv in ge... } & & 2 months ago \\
\hline \multicolumn{2}{|l|}{ howtos } & \multicolumn{3}{|c|}{ Update documentation-howto.md } & & 4 months ago \\
\hline \multicolumn{2}{|l|}{ 目 .clang-format } & \multicolumn{3}{|c|}{ raising an error in bbob2009_logger.c when best_value is NULL. Plus s... } & & a year ago \\
\hline \multicolumn{2}{|l|}{ 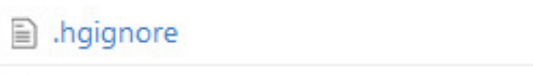 } & \multicolumn{3}{|c|}{ raising an error in bbob2009_logger.c when best_value is NULL. Plus s... } & & a year ago \\
\hline \multicolumn{2}{|l|}{ 目 AUTHORS } & \multicolumn{3}{|c|}{ small correction in AUTHORS } & & 4 months ago \\
\hline \multicolumn{2}{|l|}{ 冒 LICENSE } & \multicolumn{4}{|c|}{ Added acknowledgements to external collaborators... } & 4 months ago \\
\hline
\end{tabular}

\section{Key reatures}

- Fast, reliable implementations of many state-of-the-art multiobjective evolutionary algorithms

- Extensible with custom algorithms, problems and operators

- Supports master-slave, island-model, and hybrid parallelization

- Modular design for constructing new aptimization alooritrms from existing componers

Permissive open source license Looking for a previous release?

License

Ply documented source code

Licensed under the GNU Lesser

Ceneral Public License. 


\section{Perspectives}

\section{Challenging Open (Research) Directions}

- from algorithms to toolkits

- libraries of modules for each task (selection, variation, etc.)

- problem-specific algorithm configuration/ parameter tuning

- benchmarking

- comparison with classical approaches

- design/selection of practically relevant problems

- Algorithm/toolkit recommendations for practice

- integration of EMO and MCDM into one field

- interactive preference articulation and learning

- interactive problem design

- integration of problem-specific knowledge

\section{Questions?}




\section{Additional Slides}




\section{Instructor Biography: Dimo Brockhoff}

\section{Dimo Brockhoff}

RandOpt team

Inria Saclay - Ile-de-France

CMAP UMR 7641 École Polytechnique CNRS

Route de Saclay

91128 Palaiseau

France

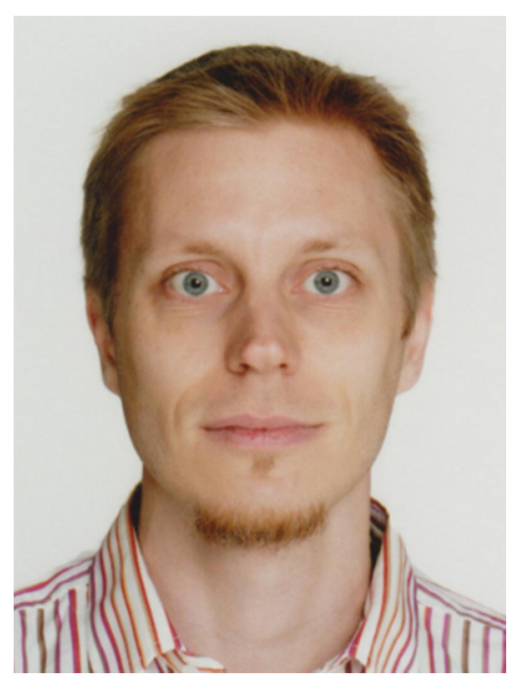

After obtaining his diploma in computer science (Dipl.-Inform.) from University of Dortmund, Germany in 2005, Dimo Brockhoff received his PhD (Dr. sc. ETH) from ETH Zurich, Switzerland in 2009. Between June 2009 and October 2011 he held postdoctoral research positions---first at Inria Saclay lle-de-France in Orsay and then at Ecole Polytechnique in Palaiseau, both in France. Since November 2011, Dimo has been a permanent researcher at Inria: from 2011 till 2016 with the Inria Lille - Nord Europe research center and since October 2016 with the Saclay - Ilede-France research center, co-located with CMAP, Ecole Polytechnique. His most recent research interests are focused on evolutionary multiobjective optimization (EMO) and other (single-objective) blackbox optimization techniques, in particular with respect to benchmarking, theoretical aspects, and expensive optimization. 


\section{References}

[Auger et al. 2009a] A. Auger, J. Bader, D. Brockhoff, and E. Zitzler. Theory of the Hypervolume Indicator: Optimal $\mu$-Distributions and the Choice of the Reference Point. In Foundations of Genetic Algorithms (FOGA 2009), pages 87-102, New York, NY, USA, 2009. ACM.

[Auger et al. 2009b] A. Auger, J. Bader, D. Brockhoff, and E. Zitzler. Articulating User Preferences in ManyObjective Problems by Sampling the Weighted Hypervolume. In G. Raidl et al., editors, Genetic and Evolutionary Computation Conference (GECCO 2009), pages 555-562, New York, NY, USA, 2009. ACM

[Bader 2010] J. Bader. Hypervolume-Based Search For Multiobjective Optimization: Theory and Methods. PhD thesis, ETH Zurich, 2010

[Bader and Zitzler 2011] J. Bader and E. Zitzler. HypE: An Algorithm for Fast Hypervolume-Based ManyObjective Optimization. Evolutionary Computation 19(1):45-76, 2011.

[Bader et al. 2009] J. Bader, D. Brockhoff, S. Welten, and E. Zitzler. On Using Populations of Sets in Multiobjective Optimization. In M. Ehrgott et al., editors, Conference on Evolutionary Multi-Criterion Optimization (EMO 2009), volume 5467 of LNCS, pages 140-154. Springer, 2009

[Bandaru and Deb 2015] S. Bandaru and K. Deb. Temporal Innovization: Evolution of Design Principles Using Multi-objective Optimization. In A. Gaspar-Cunha et al., editors, Proc. EMO 2015, volume 9018 of LNCS, pages 79-93, Springer, 2015

[Bechikh et al. 2015] S. Bechikh, M. Kessentini, L. Ben Said and K. Ghedira. Preference Incorporation in Evolutionary Multiobjective Optimization: A Survey of the State-of-the-Art. Advances in Computers, 98:141-207, 2015

[Bezerra et al. 2015] L. Bezerra, M. Lopez-lbanez, T. Stützle. To DE or Not to DE? Multi-objective Differential Evolution Revisited from a Component-Wise Prespective. In A. Gaspar-Cunha et al., editors, Proc. EMO 2015, volume 9018 of LNCS, pages 48-63, Springer, 2015

[Branke 2008] J. Branke. Consideration of Partial User Preferences in Evolutionary Multiobjective Optimization. In Multiobjective Optimization, volume 5252 of LNCS, pages 157-178. Springer, 2008 


\section{References}

[Branke and Deb 2004] J. Branke and K. Deb. Integrating User Preferences into Evolutionary Multi-Objective Optimization. In Y. Jin, editor, Knowledge Incorporation in Evolutionary Computation, pages 461-477. Springer, 2004

[Branke et al. 2014] J. Branke, S. Greco, R. Slowinski and P. Zielniewicz. Learning Value Functions in Interactive Evolutionary Multiobjective Optimization. IEEE Transactions on Evolutionary Computation, 19: 88-102, 2014

[Bringmann 2012] K. Bringmann. An improved algorithm for Klee's measure problem on fat boxes.

Computational Geometry: Theory and Applications, 45:225-233, 2012.

[Bringmann 2013] K. Bringmann. Bringing Order to Special Cases of Klee's Measure Problem. arXiv preprint arXiv:1301.7154 (2013).

[Bringmann and Friedrich 2009] K. Bringmann and T. Friedrich. Approximating the Least Hypervolume Contributor: NP-hard in General, But Fast in Practice. In M. Ehrgott et al., editors, Conference on Evolutionary Multi-Criterion Optimization (EMO 2009),pages 6-20. Springer, 2009

[Bringmann, et al. 2014] K. Bringmann, T. Friedrich, and and Patrick Klitzke. Two-dimensional subset selection for hypervolume and epsilon-indicator. Genetic and Evolutionary Computation Conference (GECCO 2014), pages 589-596. ACM, 2014

[Brockhoff et al. 2009] D. Brockhoff, T. Friedrich, N. Hebbinghaus, C. Klein, F. Neumann, and E. Zitzler. On the Effects of Adding Objectives to Plateau Functions. IEEE Transactions on Evolutionary

Computation, 13(3):591-603, 2009

[Brockhoff et al. 2012] D. Brockhoff, T. Wagner, and H. Trautmann. On the Properties of the R2 Indicator. In Genetic and Evolutionary Computation Conference (GECCO 2012), pages 465-472. ACM, 2012

[Brockhoff et al. 2013] D. Brockhoff, J. Bader, L. Thiele and E. Zitzler. Directed Multiobjective Optimization Based on the Weighted Hypervolume Indicator. Journal of Multicriteria Decision Analysis, 20(5-6):291317,2013 


\section{References}

[Brockhoff et al. 2014] D. Brockhoff, Y. Hamadi, and S. Kaci. Using Comparative Preference Statements in Hypervolume-Based Interactive Multiobjective Optimization. Learning and Intelligent Optimization (LION 2014), pages 121-136. Springer, 2014

[Brockhoff et al. 2017] D. Brockhoff, A. Auger, N. Hansen, and T. Tusar. Quantitative Performance Assessment of Multiobjective Optimizers: The Average Runtime Attainment Function. In Conference on Evolutionary Multi-Criterion Optimization (EMO 2017), pages 103-119. Springer, 2017

[Calonder et al. 2006] M. Calonder, S. Bleuler, and E. Zitzler. Module Identification from Heterogeneous Biological Data Using Multiobjective Evolutionary Algorithms. In T. P. Runarsson et al., editors, Conference on Parallel Problem Solving from Nature (PPSN IX), volume 4193 of LNCS, pages 573582. Springer, 2006

[Camerini et al. 1984] P. M. Camerini, G. Galbiati, and F. Maffioli. The complexity of multi-constrained spanning tree problems. In Theory of algorithms, Colloquium PECS 1984, pages 53-101, 1984.

[Deb and Kumar 2007] K. Deb and A. Kumar. Light Beam Search Based Multi-objective Optimization Using Evolutionary Algorithms. In Congress on Evolutionary Computation (CEC 2007), pages 2125-2132. IEEE Press, 2007

[Deb and Srinivasan 2006] K. Deb and A. Srinivasan. Innovization: Innovating Design Principles through Optimization. In Proc. GECCO 2006, pages 1629-1636. ACM, 2006

[Deb and Sundar 2006] K. Deb and J. Sundar. Reference Point Based Multi-Objective Optimization Using Evolutionary Algorithms. In Maarten Keijzer et al., editors, Conference on Genetic and Evolutionary Computation (GECCO 2006), pages 635-642. ACM Press, 2006

[Deb et al. 2014] K. Deb, S. Bandaru, D. Greiner, A. Gaspar-Cunha and C. Celal Tutum. An integrated approach to automated innovization for discovering useful design principles: Case studies from engineering. Applied Soft Computing, 15:42-56, 2014

[Díaz-Manríquez et al. 2013] A. Díaz-Manríquez, G. Toscano-Pulido, C. A. C. Coello and R. Landa-Becerra. A ranking method based on the $\mathrm{R} 2$ indicator for many-objective optimization. In IEEE Congress on Evolutionary Computation (CEC), pages 1523-1530. IEEE. 


\section{References}

[Emmerich et al. 2007] M. Emmerich, A. Deutz and N. Beume. Gradient-Based/Evolutionary Relay Hybrid for Computing Pareto Front Approximations Maximizing the S-Metric. In Bartz-Beielstein et al., editors, Proc. Hybrid Metaheuristics, pages 140-156. Springer, 2007

[Fonseca and Fleming 1998a] C. M. Fonseca and Peter J. Fleming. Multiobjective Optimization and Multiple Constraint Handling with Evolutionary Algorithms-Part I: A Unified Formulation. IEEE Transactions on Systems, Man, and Cybernetics, 28(1):26-37, 1998

[Fonseca and Fleming 1998b] C. M. Fonseca and Peter J. Fleming. Multiobjective Optimization and Multiple Constraint Handling with Evolutionary Algorithms-Part II: Application Example. IEEE Transactions on Systems, Man, and Cybernetics, 28(1):38-47, 1998

[Fonseca et al. 2011] C. M. Fonseca, A. P. Guerreiro, M. López-lbáñez, and L. Paquete. On the computation of the empirical attainment function. In Takahashi et al., editors, Proc. EMO, volume 6576 of LNCS, pages 106-120. Springer, 2011

[Friedrich et al. 2011] T. Friedrich, K. Bringmann, T. Voß, C. Igel. The Logarithmic Hypervolume Indicator. In Beyer and Langdon, editors, Proc. FOGA. ACM, 2011.

[Guerreiro et al. 2015] A. P. Guerreiro, C. M. Fonseca, and L. Paquete. Greedy Hypervolume Subset Selection in the Three-Objective Case. In Genetic and Evolutionary Computation Conference (GECCO 2015), pages 671-678. ACM, 2015

[Guerreiro and Fonseca 2017] A. P. Guerreiro and C. M. Fonseca. Computing and updating hypervolume contributions in up to four dimensions. IEEE Transactions on Evolutionary Computation (2017).

[Greiner et al. 2007] D. Greiner, J. M. Emperador, G. Winter, and B. Galván. Improving Computational Mechanics Optimium Design Using Helper Objectives: An Application in Frame Bar Structures. In Conference on Evolutionary Multi-Criterion Optimization (EMO 2007), volume 4403 of LNCS, pages 575589. Springer, 2007

[Hadka and Reed 2013] D. Hadka and P. Reed. Borg: An Auto-Adaptive Many-Objective Evolutionary Computing Framework. Evolutionary Computation, 21(2):231-259, 2013 


\section{References}

[Handl et al. 2008a] J. Handl, S. C. Lovell, and J. Knowles. Investigations into the Effect of Multiobjectivization in Protein Structure Prediction. In G. Rudolph et al., editors, Conference on Parallel Problem Solving From Nature (PPSN X), volume 5199 of LNCS, pages 702-711. Springer, 2008

[Handl et al. 2008b] J. Handl, S. C. Lovell, and J. Knowles. Multiobjectivization by Decomposition of Scalar Cost Functions. In G. Rudolph et al., editors, Conference on Parallel Problem Solving From Nature (PPSN X), volume 5199 of LNCS, pages 31-40. Springer, 2008

[lgel et al. 2007] C. Igel, N. Hansen, and S. Roth. Covariance Matrix Adaptation for Multi-objective Optimization. Evolutionary Computation, 15(1):1-28, 2007

[Jaszkiewicz and Branke 2008] A. Jaszkiewicz and J. Branke. Interactive Multiobjective Evolutionary Algorithms. In: Multiobjective Optimization: Interactive and Evolutionary Approaches, pages 179-193, Springer, 2008

[Jensen 2004] M. T. Jensen. Helper-Objectives: Using Multi-Objective Evolutionary Algorithms for SingleObjective Optimisation. Journal of Mathematical Modelling and Algorithms, 3(4):323-347, 2004

[Judt et al. 2011] L. Judt, O. Mersmann, and B. Naujoks. Non-monotonicity of obtained hypervolume in 1greedy S-Metric Selection. In: Conference on Multiple Criteria Decision Making (MCDM 2011), 2011

[Krause et al. 2016] O. Krause, T. Glasmachers, N. Hansen, and C. Igel. Unbounded population MO-CMAES for the bi-objective BBOB test suite. In Companion of the 18th International Conference on Genetic and Evolutionary Computation (GECCO 2012): BBOB-2016 Workshop, pages 1177-1184, ACM, 2016

[Knowles et al. 2001] J. D. Knowles, R. A. Watson, and D. W. Corne. Reducing Local Optima in SingleObjective Problems by Multi-objectivization. In E. Zitzler et al., editors, Conference on Evolutionary Multi-Criterion Optimization (EMO 2001), volume 1993 of LNCS, pages 269-283. Springer, 2001

[Kuhn et al. 2014] T. Kuhn, C. M. Fonseca, L. Paquete, S. Ruzika, and J. R. Figueira. Hypervolume subset selection in two dimensions: Formulations and algorithms. Technical report. Technische Universität Kaiserslautern, Fachbereich Mathematik, 2014 


\section{References}

[López-lbáñez et al 2010] M. López-lbáñez, L. Paquete, and T. Stützle. Exploratory Analysis of Stochastic Local Search Algorithms in Biobjective Optimization. In T. Bartz-Beielstein, M. Chiarandini, L. Paquete, and M. Preuss, editors. Experimental Methods for the Analysis of Optimization Algorithms, pages 209-222. Springer, 2010

[Lopez-lbanez and Knowles 2015] M. Lopez-lbanez and J. D. Knowles. Machine Decision Makers as a Laboratory for Interactive EMO. In A. Gaspar-Cunha et al., editors, Proc. EMO, volume 9019 of LNCS, pages 295-309. Springer, 2015

[Miettienen 1999] K. Miettinen. Nonlinear Multiobjective Optimization. Kluwer, Boston, MA, USA, 1999

[Neumann and Wegener 2006] F. Neumann and I. Wegener. Minimum Spanning Trees Made Easier Via Multi-Objective Optimization. Natural Computing, 5(3):305-319, 2006

[Obayashi and Sasaki 2003] S. Obayashi and D. Sasaki. Visualization and Data Mining of Pareto Solutions Using Self-Organizing Map. In Conference on Evolutionary Multi-Criterion Optimization (EMO 2003), volume 2632 of LNCS, pages 796-809. Springer, 2003

[Sacks et al. 1989] J. Sacks, W. Welch, T. Mitchell, H. Wynn. : Design and Analysis of Computer Experiments. Statistical Science, 4(4):409-423, 1989

[Schaffer 1985] J. D. Schaffer. Multiple Objective Optimization with Vector Evaluated Genetic Algorithms. In John J. Grefenstette, editor, Conference on Genetic Algorithms and Their Applications, pages 93-100, 1985.

[Segura et al. 2013] C. Segura, C. A. Coello Coello, M. Gara and L. Coromoto. Using multi-objective evolutionary algorithms for single-objective optimization. In: 4OR, 11(3):201-228. Springer, 2013.

[Siegfried et al. 2009] T. Siegfried, S. Bleuler, M. Laumanns, E. Zitzler, and W. Kinzelbach. Multi-Objective Groundwater Management Using Evolutionary Algorithms. IEEE Transactions on Evolutionary Computation, 13(2):229-242, 2009

[Tanino et al. 1993] T. Tanino, M. Tanaka, and C. Hojo. An Interactive Multicriteria Decision Making Method by Using a Genetic Algorithm. In: Conference on Systems Science and Systems Engineering, pages 381-386, 1993 


\section{References}

[Thiele et al. 2002] L. Thiele, S. Chakraborty, M. Gries, and S. Künzli. Design Space Exploration of Network Processor Architectures. In Network Processor Design 2002: Design Principles and Practices. Morgan Kaufmann, 2002

[Trautmann et al. 2013] H. Trautmann, T. Wagner, and D. Brockhoff. R2-EMOA: Focused Multiobjective Search Using R2-Indicator-Based Selection. Learning and Intelligent Optimization Conference (LION 2013), pages 70-74, Springer, 2013. Short paper.

[Ulrich et al. 2007] T. Ulrich, D. Brockhoff, and E. Zitzler. Pattern Identification in Pareto-Set Approximations. In M. Keijzer et al., editors, Genetic and Evolutionary Computation Conference (GECCO 2008), pages 737-744. ACM, 2008.

[Verel et al. 2011] S. Verel, C. Dhaenens, A. Liefooghe. Set-based Multiobjective Fitness Landscapes: A Preliminary Study. In Genetic and Evolutionary Computation Conference (GECCO 2011), pages 769776. ACM, 2011.

[Voß et al. 2010] T. Voß, N. Hansen, and C. Igel. Improved Step Size Adaptation for the MO-CMA-ES. In J. Branke et al., editors, Genetic and Evolutionary Computation Conference (GECCO 2010), pages 487494. ACM, 2010

[Wagner et al. 2008] T. Wagner, D. Passmann, K. Weinert, D. Biermann and A. Bledzki. Efficient Modeling and Optimization of the Property Gradation of Self-Reinforced Polypropylene Sheets within a ThermoMechanical Compaction Process. In R. Teti, editor, Proc. ICME, pages 447-452. Edizione Ziino, 2008

[Wagner et al. 2010] T. Wagner, M. Emmerich, A. Deutz and W. Ponweiser. Improvement Criteria for Model-Based Multi-Objective Optimization. In R. Schaefer et al., editors, Proc. PPSN, volume 6238 of LNCS, pages 718-727. Springer, 2010

[Watanabe and Sakakibara 2007] S. Watanabe and K. Sakakibara. A multiobjectivization approach for vehicle routing problems. In Conference on Evolutionary Multi-Criterion Optimization (EMO 2007), volume 4403 of LNCS, pages 660-672. Springer, 2007 


\section{References}

[Weinert et al. 2009] K. Weinert, A. Zabel, P. Kersting, T. Michelitsch and T. Wagner. On the Use of Problem-Specic Candidate Generators for the Hybrid Optimization of Multi-Objective Production Engineering Problems. Evolutionary Computation, 17(4):527-544, 2009

[Yildiz and Suri 2012] H. Yildiz and S. Suri. On Klee's measure problem for grounded boxes. Proceedings of the 2012 symposium on Computational Geometry. ACM, 2012

[Zhang and Li 2007] Q. Zhang and H. Li. MOEA/D: A Multiobjective Evolutionary Algorithm Based on Decomposition. IEEE Transactions on Evolutionary Computation, 11(6):712--731, 2007

[Zhang et al. 2008] Q. Zhang, A. Zhou and Y. Jin. RM-MEDA: A Regularity Model-Based Multiobjective Estimation of Distribution Algorithm. IEEE Transactions on Evolutionary Computation, 12(1):41-63, 2008

[Zhang et al. 2012] L. Zhang, T. Wagner and D. Biermann. Optimization of Cutting Parameters for Drilling Nickel-Based Alloys using Statistical Experimental Design Techniques. In S. Hinduja and L. Li, editors, Proc. MATADOR, pages 123-126. Springer, 2012

[Zitzler 1999] E. Zitzler. Evolutionary Algorithms for Multiobjective Optimization: Methods and Applications. PhD thesis, ETH Zurich, Switzerland, 1999

[Zitzler and Künzli 2004] E. Zitzler and S. Künzli. Indicator-Based Selection in Multiobjective Search. In X. Yao et al., editors, Conference on Parallel Problem Solving from Nature (PPSN VIII), volume 3242 of LNCS, pages 832-842. Springer, 2004

[Zitzler et al. 2000] E. Zitzler, K. Deb, and L. Thiele. Comparison of Multiobjective Evolutionary Algorithms: Empirical Results. Evolutionary Computation, 8(2):173-195, 2000

[Zitzler et al. 2003] E. Zitzler, L. Thiele, M. Laumanns, C. M. Fonseca, and V. Grunert da Fonseca. Performance Assessment of Multiobjective Optimizers: An Analysis and Review. IEEE Transactions on Evolutionary Computation, 7(2):117-132, 2003

[Zitzler et al. 2010] E. Zitzler, L. Thiele, and J. Bader. On Set-Based Multiobjective Optimization. IEEE Transactions on Evolutionary Computation, 14(1):58-79, 2010 


\section{Acknowledgements}

I would like to thank in particular Eckart Zitzler and Tobias Wagner who contributed significantly to the content of these slides over the years.

Many thanks go also to Carlos Fonseca and Manuel Lópezlbáñez for pointing out some mistakes during my PPSN 2016 presentation on the same topic that should be corrected in these slides. 